\title{
Semi-analytical approach to criteria for ignition of excitation waves
}

\author{
B. Bezekci, ${ }^{1}$ I. Idris, ${ }^{2}$ R. D. Simitev, ${ }^{3}$ and V. N. Biktashev ${ }^{1, *}$ \\ ${ }^{1}$ College of Engineering, Mathematics and Physical Sciences, University of Exeter, Exeter EX4 4QF, UK \\ ${ }^{2}$ Mathematical Sciences, Bayero University, Kano, Nigeria \\ ${ }^{3}$ School of Mathematics and Statistics, University of Glasgow, Glasgow G12 8QW, UK
}

\begin{abstract}
We consider the problem of ignition of propagating waves in one-dimensional bistable or excitable systems by an instantaneous spatially extended stimulus. Earlier we proposed a method (Idris \& Biktashev, PRL, vol 101, 2008, 244101) for analytical description of the threshold conditions based on an approximation of the (center-)stable manifold of a certain critical solution. Here we generalize this method to address a wider class of excitable systems, such as multicomponent reaction-diffusion systems and systems with non-self-adjoint linearized operators, including systems with moving critical fronts and pulses. We also explore an extension of this method from a linear to a quadratic approximation of the (center-)stable manifold, resulting in some cases in a significant increase in accuracy. The applicability of the approach is demonstrated on five test problems ranging from archetypal examples such as the Zeldovich-Frank-Kamenetsky equation to near realistic examples such as the Beeler-Reuter model of cardiac excitation. While the method is analytical in nature, it is recognised that essential ingredients of the theory can be calculated explicitly only in exceptional cases, so we also describe methods suitable for calculating these ingredients numerically.
\end{abstract}

PACS numbers: 87.10.-e, 82.40.Ck, 02.90.+p

\section{Contents}

I. Introduction
A. Motivation
B. Problem formulation
C. Aims

II. Methods

A. Linear approximation of the center-stable manifold: principal approach

B. Linear approximation of the strength-extent curve

1. General setting

2. The case of critical nucleus

3. The case of moving critical solution

C. Quadratic approximation of the stable manifold

D. Hybrid approach: numerical computation of functions required by the analytical theory 9

1. Rationale

2. The case of critical nucleus

9

3. The case of moving critical solution

E. A priori bound in the critical nucleus case

III. Zeldovich - Frank-Kamenetsky equation 12

A. Model formulation

B. The small-threshold limit and the "fully analytical" result

C. Hybrid approach

D. Quadratic theory

9
9
10
11

IV. McKean equation
A. Model formulation
B. Linear theory
C. Quadratic theory

V. The caricature model of the $I_{\mathrm{Na}}$-driven cardiac excitation front
A. Model formulation
B. Linearized problem and eigenfunctions
C. Strength-extent curve

VI. FitzHugh-Nagumo system
A. Model formulation
B. Linear theory

VII. Modified Beeler-Reuter model of cardiac excitation
A. Model formulation
B. Linear theory

VIII. Discussion

Acknowledgements

Appendices

A. On "frozen nuclei" in the McKean equation

B. On linearization of discontinuous right-hand sides

C. Finite element discretization for the McKean model

D. The modified Beeler-Reuter model

References 


\section{INTRODUCTION}

\section{A. Motivation}

An excitable system is a nonlinear active system that has a stable resting state, so that a weak, sub-threshold stimulus causes a straightforward return to the rest, but a stronger, over-threshold stimulus can produce a significant, qualitatively different response. When such a system is spatially distributed, response to an over-threshold stimulus has the form of a propagating excitation wave in a shape of a non-decaying pulse, and one usually speaks about an excitable medium. A closely related concept is a trigger wave in a bistable medium: this takes place when the medium does not completely recover after a pulse but switches into a different steady state; trigger waves also often occur as idealizations of fronts or backs of excitation pulses. Excitable and bistable systems are widespread in nature and technology. Historically, the concept of excitability was first introduced in biology for nerve cells, and then applied to their electronic analogues. Later it was extended also to many other types of biological waves of signalling and in population dynamics, as well as such diverse physical situations as combustion and other chemical reaction waves, self-heating in metals and superconductors, phase transitions, domain wall movement in liquid crystals, nonlinear optics, surface boiling and laminar-turbulent transition in fluid flows, to name a few. See e.g. [1-10] for some literature on the topic

It is often important not only to know that a particular system can support a non-decaying propagating wave, but also to know what initial conditions can lead to it. The threshold character of the response of excitable and bistable systems, which characterizes already their local dynamics, gets much more complicated in the spatially extended context: the outcome of the localized perturbation will depend on its spatial and temporal characteristics as well as on its magnitude and modality. The conditions for initiation of propagating waves can be very important in practical applications. For instance, in the heart, excitation waves trigger coordinated contraction of the muscle and the failure of initiation can cause or contribute to serious or fatal medical conditions, or render inefficient the work of pacemakers or defibrillators [1]. In combustion, understanding of initiation is of critical importance for safety during the storage and transport of combustible materials [12. In several key industrial processes, involving heat-generating elements, an important safety concern is the boiling crisis, or transition between a low-temperature and a high-temperature regimes [13], which can proceed via trigger fronts [14.

\section{B. Problem formulation}

We consider a formulation of the problems of initiation of propagating waves in terms of one-dimensional reaction-diffusion system,

$$
\frac{\partial \mathbf{u}}{\partial t}=\mathbf{D} \frac{\partial^{2} \mathbf{u}}{\partial x^{2}}+\mathbf{f}(\mathbf{u})
$$

where $\mathbf{u}(x, t): \mathbb{R} \times \mathbb{R} \rightarrow \mathbb{R}^{d}$ is a $d$-component reagents field, $d \geq 1$, defined for $x \in \mathbb{R}$ and $t \in \mathbb{R}_{+}$, vectorfunction $\overline{\mathbf{f}}: \mathbb{R}^{d} \rightarrow \mathbb{R}^{d}$ describes the reaction rates and $\mathbf{D} \in \mathbb{R}^{d \times d}$ is the matrix of diffusivity. We assume that this system has an asymptotically stable spatially uniform equilibrium, called resting state,

$$
\mathbf{u}(x, t)=\mathbf{u}_{r}, \quad \mathbf{f}\left(\mathbf{u}_{r}\right)=\mathbf{0},
$$

and an orbitally stable family of propagating wave solutions of the form

$$
\begin{array}{r}
\mathbf{u}(x, t)=\mathbf{u}_{\mathrm{w}}\left(x-c_{\mathrm{w}} t-s_{\mathrm{w}}\right), \\
\mathbf{u}_{\mathrm{w}}(\infty)=\mathbf{u}_{r}, \mathbf{u}_{\mathrm{w}}(-\infty)=\mathbf{u}_{-},
\end{array}
$$

where $\mathbf{u}_{-}$is also a stable spatially uniform equilibrium, $\mathbf{f}\left(\mathbf{u}_{-}\right)=\mathbf{0}$, which may or may not coincide with $\mathbf{u}_{r}$. When $\mathbf{u}_{-}=\mathbf{u}_{r}$ the propagating wave solution is known as a propagating pulse, otherwise we shall call it a propagating front, and refer to $\mathbf{u}_{-}$as the post-front equilibrium; then $\mathbf{u}_{r}$ may also be called the pre-front equilibrium. In (3), $c_{\mathrm{w}}>0$ is a fixed constant, the wave propagation speed, and $s_{\mathrm{w}}$ is an arbitrary constant, the parameter of the family. Roughly speaking we assume that (3) and (2) are the only attractors within the part of the phase space of (1) that is of practical interest, and we seek to find the boundary of the basins of attraction of $\mathbf{u}_{\mathrm{w}}$.

In these terms, we seek to describe the localized (in space and time) perturbations of the resting state $\mathbf{u}_{r}$ which can lead to the propagating wave solutions $\mathbf{u}_{\mathrm{w}}$. A localized perturbation will in fact typically generate two waves propagating away from the perturbed site in the opposite directions; this is obvious for perturbations that are even functions of $x$. With that in mind, we aim at classification of the solutions of the system (1) set on $x \in[0, \infty), t \in[0, \infty)$, supplied with the following initial and boundary conditions,

$$
\begin{aligned}
& \mathbf{u}(x, 0)=\mathbf{u}_{0}(x)=\mathbf{u}_{r}+\mathbf{u}_{\mathrm{s}}(x), \quad x>0, \\
& \mathbf{D u}_{x}(0, t)=-\mathbf{I}_{\mathrm{s}}(t), \quad t>0,
\end{aligned}
$$

in terms of their behaviour as $t \rightarrow \infty$ : whether it will approach the propagating wave solution ("ignition") or the resting state ("failure"), as illustrated in fig. 1(a,b) [56]. The functions $\mathbf{u}_{\mathrm{s}}(x)$ and $\mathbf{I}_{\mathrm{s}}(t)$ are assumed to have a finite support, $\mathbf{u}_{\mathrm{s}}(x) \equiv \mathbf{0}$ for $x>x_{\mathrm{s}}$, and $\mathbf{I}_{\mathrm{s}}(t) \equiv \mathbf{0}$ for $t>t_{\mathrm{s}}$.

A typical formulation is when only one of $\mathbf{u}_{\mathbf{s}}(\cdot)$ and $\mathbf{I}_{\mathrm{S}}(\cdot)$ is nonzero. If the dependence is on just one parameter, then one speaks about threshold value(s) of the parameter, separating the two outcomes. When there are two parameters, one can talk about a threshold curve, or a critical curve, see fig. 1(c). The simplest and standard formulations are: 


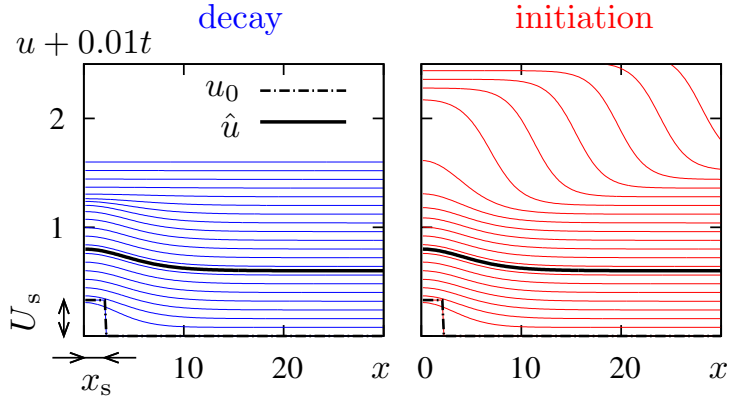

(a)

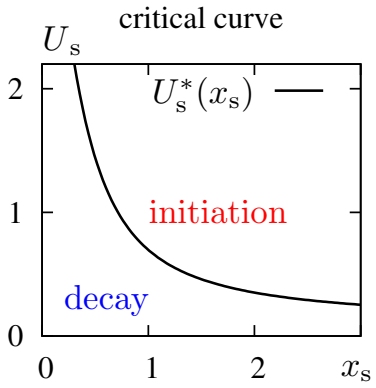

(c)

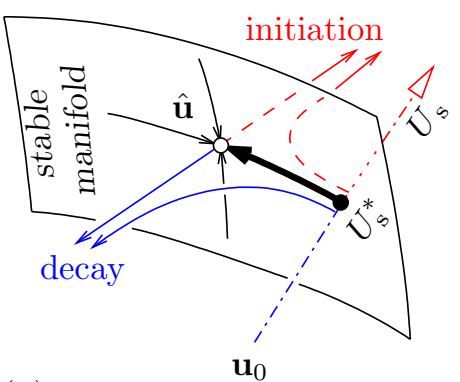

(d)

FIG. 1: (Color online) (a,b) Response to a below- and above-threshold initial perturbation in ZFK equation, given by formulas

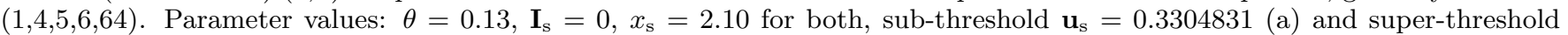
$\mathbf{u}_{\mathrm{s}}=0.3304833$ (b) cases, numerics using central difference centered in space with step $\Delta_{x}=0.15$ and forward Euler in time with step $\Delta_{t}=0.01$. Dash-dotted black lines: initial conditions, bold solid black lines: the critical nuclei. (c) The corresponding critical strength-extent curve, separating ignition initial conditions from decay initial conditions. (d) Sketch of a stable manifold of the critical solution for the ZFK equation. The critical nucleus is represented by the empty circle; the critical trajectories, constituting the center-stable manifold, are shown in thin solid black lines. The family of initial conditions is represented by the dash-dotted lines. The bold solid black line is the critical trajectory with initial condition in that family. The sub-threshold trajectories are represented by the thin blue lines, while the thin red lines represent super-threshold trajectories. Note that the point where the initial condition intersect the center-stable manifold is shown as the filled circle.

- "Stimulation by voltage":

$$
\mathbf{I}_{\mathrm{s}}(t)=\mathbf{0}, \quad \mathbf{u}_{\mathrm{s}}(x)=U_{\mathrm{s}} \mathbf{X}(x) .
$$

That is, the initial condition is the resting state $\mathbf{u}_{r}$, displaced by the magnitude defined by the parameter $U_{\mathrm{s}}$ with a normalized spatial profile defined by $\mathbf{X}(x)$. In all specific examples we shall use simply a rectangular profile of a width $x_{\mathrm{s}}$,

$$
\mathbf{X}(x)=\mathrm{H}\left(x_{\mathrm{s}}-x\right) \mathbf{e},
$$

where $\mathrm{H}(\cdot)$ is the Heaviside step function and $\mathbf{e} \in$ $\mathbb{R}^{d}$ is a constant vector defining the modality of the perturbation. Then the critical curve can be considered in the plane $\left(x_{\mathrm{s}}, U_{\mathrm{s}}\right)$.

- "Stimulation by current":

$$
\mathbf{u}_{\mathrm{s}}(x)=\mathbf{0}, \quad \mathbf{I}_{\mathrm{s}}(t)=I_{\mathrm{s}} \mathbf{T}(t) .
$$

That is, the initial condition is the unperturbed resting state, but there is a constant current injected through the left boundary of the interval, where $I_{\mathrm{s}}$ defines the strength of the current and $\mathbf{T}(t)$ its normalized temporal profile. The most popular formulation is that of a rectangular profile of duration $t_{\mathrm{s}}$,

$$
\mathbf{T}(t)=\mathrm{H}\left(t_{\mathrm{s}}-t\right) \mathbf{e},
$$

where the fixed vector $\mathbf{e}$ determines which reagent(s) are being injected. The critical curve will then be in the plane $\left(t_{\mathrm{s}}, I_{\mathrm{s}}\right)$.

Historically, the "stimulation by current" formulation has been most popular with electrophysiologists and a standard term for the critical curve $\left(t_{\mathrm{s}}, I_{\mathrm{s}}\right)$ is the "strengthduration curve". We are not aware of any standard term for the critical curve $\left(x_{\mathrm{s}}, U_{\mathrm{s}}\right)$; by analogy with the other case we shall call it the "strength-extent curve".

We find it convenient to formalize the initiation problem as one posed on the whole real line $x \in \mathbb{R}$,

$$
\begin{aligned}
& \frac{\partial \mathbf{u}}{\partial t}=\mathbf{D} \frac{\partial^{2} \mathbf{u}}{\partial x^{2}}+\mathbf{f}(\mathbf{u})+\mathbf{h}(x, t), \quad(x, t) \in \mathbb{R} \times \mathbb{R}_{+}, \\
& \mathbf{u}(x, 0)=\mathbf{u}_{r}+\mathbf{u}_{\mathrm{s}}(x), \quad \mathbf{h}(x, t) \equiv \mathbf{0} \text { for } t>t_{\mathrm{s}} .
\end{aligned}
$$

where the initial condition is an even continuation of the one in (4),

$$
\mathbf{u}_{\mathrm{s}}(-x) \equiv \mathbf{u}_{\mathrm{s}}(x)= \begin{cases}U_{\mathrm{s}} \mathbf{X}(x), & x \geq 0 \\ U_{\mathrm{s}} \mathbf{X}(-x), & x<0\end{cases}
$$

and the boundary condition at $x=0$ in (4) is formally represented by the source term

$$
\mathbf{h}(x, t)=2 I_{\mathrm{s}} \mathbf{T}(t) \delta(x) .
$$

where $\delta(\cdot)$ is the Dirac delta function.

\section{Aims}

Mathematically, the problem of determining the conditions of initiation of propagating waves in excitable or bistable media is spatially-distributed, nonstationary, nonlinear and has generally no helpful symmetries, so the accurate treatment is feasible only numerically. However, the practical value of these conditions is so high that analytical answers, even if very approximate, are in high demand. Historically, there have been numerous attempts to obtain such answers, based on various phenomenological and heuristic approaches, e.g. [15] 19]. 
The motivation for our present approach may be traced to the results by McKean and Moll [20] and Flores [21], who established that the boundary in the space of initial data of (1) between the basins of attraction of (2) and (3) is a stable manifold of a certain "standing wave" solution, later also known as the critical nucleus. The critical nucleus is a solution of (1) which is a bounded, non-constant function of $x$, independent of $t$ and is unstable, with one positive eigenvalue. The appearance of such a critical nucleus solution and its role is illustrated in fig. 1, if the initial data are very near the threshold between ignition and decay, the critical nucleus appears as a long transient before the outcome becomes apparent, and this does not depend on the sort of initial data, as long as they are near the threshold. This understanding has led to attempts to describe the critical conditions using Galerkin style approximations 22, with analytical answers obtainable by subsequent linearization [23. This idea of the stable manifold of the critical solution has also been used to develop sophisticated numerical schemes for describing the critical conditions [24. We have demonstrated that linearization of the stable manifold without any Galerkin projection but directly in the functional space produces surprisingly good reasults for a simple bistable model [25].

In the present paper, we seek to further explore and extend the method of [25]. We focus on the case of stimulation by voltage and the strength-extent curve, leaving the stimulation by current and the strength-duration curve to other publications (note though that a simple case of the strength-duration curve was considered in [25]). In the case of stimulation by voltage, the mathematical problem is one about the basin of attraction of a dynamic system in a functional space. We investigate how the quality of approximation produced by our method depends on the parameters that define various test systems. Moreover, we investigate the feasibility of improving the accuracy by using a quadratic rather than a linear approximation of the critical manifold, and related problems. Finally, we extend the method to the case where there are no critical nucleus solutions. This is observed in multicomponent reaction-diffusion systems, where it has been previously demonstrated that instead of a critical nucleus, one has unstable propagating waves, such as critical pulses [26] or critcal fronts [27].

The structure of the paper is as follows. In Section II we describe the proposed analytical methods, including both the linear and the quadratic approximations of the critical manifold for the case of the critical nucleus, and the linear approximation for the case of moving critical solutions, as well as the (rather standard) numerical methods used in the study. Subsequent sections are dedicated to specific examples of application of the described method. In Section III, we consider the onecomponent reaction-diffusion equation with cubic nonlinearity, known as the Zeldovich-Frank-Kamenetsky (ZFK) equation, or the Nagumo equation, or the Schlögl model; this section recovers relevant results from 25 and fur- ther investigates the parametric dependencies and the quadratic approximation for a model of a propagating front. In Section IV the same is done to a piece-wise linear analogue of the ZFK equation, known as the McKean equation. The piece-wise linearity of this equation means that some results can be obtained in closed form, where it was not possible in the ZFK case. Another special feature of this equation is that its right-hand side is discontinuous, which presents certain technical challenges. The subsequent three sections are dedicated to examples with moving critical solutions. Section $\mathrm{V}$ presents results in a two-component model where the critical solution is a moving front. It is a caricature model of cardiac excitation propagating fronts, and shares with the McKean equation the advantage of being piecewiselinear and of admitting analytical treatment, and also the challenge of having discontinous right-hand sides. Sections $\mathrm{VI}$ and VII are dedicated to two models where the critical solution is a pulse. Section $\mathrm{VI}$ is an application to the FitzHugh-Nagumo (FHN) system which is a classical "conceptual" model of excitable media, while Section VII considers a variant of the detailed Beeler-Reuter (BR) ionic model of cardiac excitation, which, although not being physiologically precise from a modern viewpoint, includes many features of the up-to-date realistic cardiac excitation models. Both FHN and BR models do not admit full analytical treatment and we present the result of a hybrid approach, where the ingredients of the linearized theory are obtained numerically. We conclude by discussing the results and future directions in Section VIII

\section{METHODS}

\section{A. Linear approximation of the center-stable manifold: principal approach}

We seek a classification of the outcomes in problem 9) depending on the parameters of the initial conditions (10), with $\mathbf{h}(x, t) \equiv 0$.

The principal assumption of our approach is the existence of a critical solution, which is defined as a selfsimilar solution,

$$
\begin{array}{r}
\mathbf{u}(x, t)=\hat{\mathbf{u}}(x-c t), \\
\mathbf{0}=\mathbf{D} \frac{\mathrm{d}^{2} \hat{\mathbf{u}}}{\mathrm{d} \xi^{2}}+c \frac{\mathrm{d} \hat{\mathbf{u}}}{\mathrm{d} \xi}+\mathbf{f}(\hat{\mathbf{u}}), \\
\hat{\mathbf{u}}(\infty)=\mathbf{u}_{r}, \quad \hat{\mathbf{u}}(-\infty)=\hat{\mathbf{u}}_{-}
\end{array}
$$

(where $\hat{\mathbf{u}}_{-}$may be different from $\mathbf{u}_{-}$but in our examples $\hat{\mathbf{u}}_{-}=\mathbf{u}_{r}$ when $\mathbf{u}_{-}=\mathbf{u}_{r}$ ) which, unlike the propagating wave $\mathbf{u}_{\mathrm{w}}$ defined by (3), is unstable with one unstable eigenvalue. Naturally, the speed $c$ of the critical solution is also entirely different from the speed $c_{\mathrm{w}}$ of the stable wave solution.

Similar to the stable wave solution, there is then a 
whole one-parametric family of critical solutions,

$$
\hat{\mathbf{u}}(x-c t-s), \quad s \in \mathbb{R} .
$$

Due to this translation invariance, this solution always has one zero eigenvalue. Hence its stable manifold has codimension two, whereas its center-stable manifold has codimension one and as such it can partition the phase space, i.e. it can serve as a boundary between the basins of different attractors. Our strategy is to approximate this center-stable space. In the first instance, we consider the following linear approximation.

Let us rewrite the reaction-diffusion system (RDS) (1) in a frame of reference moving with a constant speed $c$, so that $\mathbf{u}(x, t)=\tilde{\mathbf{u}}(\xi, \tau), \xi=x-c t-s, \tau=t$,

$$
\begin{aligned}
& \frac{\partial \tilde{\mathbf{u}}}{\partial \tau}=\mathbf{D} \frac{\partial^{2} \tilde{\mathbf{u}}}{\partial \xi^{2}}+c \frac{\partial \tilde{\mathbf{u}}}{\partial \xi}+\mathbf{f}(\tilde{\mathbf{u}}), \\
& \tilde{\mathbf{u}}(\xi, 0)=\mathbf{u}_{r}+\mathbf{u}_{\mathrm{s}}(\xi+s)
\end{aligned}
$$

We linearize this equation on the critical solution, which is stationary in the moving frame

$$
\tilde{\mathbf{u}}(\xi, \tau)=\hat{\mathbf{u}}(\xi)+\mathbf{v}(\xi, \tau) .
$$

The linearization gives

$$
\begin{aligned}
& \frac{\partial \mathbf{v}}{\partial \tau}=\mathbf{D} \frac{\partial^{2} \mathbf{v}}{\partial \xi^{2}}+c \frac{\partial \mathbf{v}}{\partial \xi}+\mathbf{F}(\xi) \mathbf{v}, \\
& \mathbf{v}(\xi, 0)=\mathbf{u}_{r}+\mathbf{u}_{\mathrm{s}}(\xi+s)-\hat{\mathbf{u}}(\xi),
\end{aligned}
$$

where

$$
\mathbf{F}(\xi)=\left.\frac{\partial \mathbf{f}}{\partial \mathbf{u}}\right|_{\mathbf{u}=\hat{\mathbf{u}}(\xi)}
$$

is the Jacobian of the kinetic term, evaluated at the critical solution.

Equation (15) is a linear non-homogeneous equation, with a time-independent linear operator,

$$
\partial_{\tau} \mathbf{v}=\mathcal{L} \mathbf{v}+\tilde{\mathbf{h}}, \quad \mathcal{L} \triangleq \mathbf{D} \frac{\partial^{2}}{\partial \xi^{2}}+c \frac{\partial}{\partial \xi}+\mathbf{F}(\xi) .
$$

For the sake of simplicity, let us assume that the eigenfunctions of $\mathcal{L}$,

$$
\mathcal{L} \mathbf{V}_{j}(\xi)=\lambda_{j} \mathbf{V}_{j}(\xi)
$$

are simple and form a basis in an appropriate functional space, and the same is true for the adjoint $\mathcal{L}^{+}$[57]. Another assumption, which simplifies formulas and is true for all examples considered, is that all eigenvalues important for the theory are real. We shall enumerate the eigenpairs in the decreasing order of $\lambda_{j}$, so by assumption we always have $\lambda_{1}>\lambda_{2}=0>\lambda_{3}>\ldots$

Then the general solution of problem 15 in that space can be written as a generalized Fourier series

$$
\mathbf{v}(\xi, \tau)=\sum_{j} a_{j}(\tau) \mathbf{V}_{j}(\xi)
$$

The coefficients $a_{j}$ will then satisfy decoupled ODEs,

$$
\frac{\mathrm{d} a_{j}}{\mathrm{~d} \tau}=\lambda_{j} a_{j}
$$

where

$$
a_{j}(0)=\left\langle\mathbf{W}_{j}(\xi) \mid \mathbf{v}(\xi, 0)\right\rangle
$$

the scalar product $\langle\cdot \mid \cdot\rangle$ is defined as

$$
\langle\mathbf{a} \mid \mathbf{b}\rangle=\int_{-\infty}^{\infty} \overline{\mathbf{a}}^{\top} \mathbf{b} \mathrm{d} \xi
$$

and $\mathbf{W}_{j}$ are eigenfunctions of the adjoint operator,

$$
\mathcal{L}^{+} \mathbf{W}_{j}=\lambda_{j} \mathbf{W}_{j}, \quad \mathcal{L}^{+}=\mathbf{D}^{\top} \frac{\partial^{2}}{\partial \xi^{2}}-c \frac{\partial}{\partial \xi}+\mathbf{F}^{\top}(\xi),
$$

which are normalized so that

$$
\left\langle\mathbf{W}_{j} \mid \mathbf{V}_{k}\right\rangle=\delta_{j, k} .
$$

The solution of 20 is

$$
a_{j}(\tau)=\mathrm{e}^{\lambda_{j} \tau} a_{j}(0) .
$$

By assumption, $\lambda_{1}>0$, and due to the translational symmetry, $\lambda_{2}=0$, and the rest of the spectrum is assumed within the left half-plane. Hence the condition of criticality is

$$
a_{1}(0)=0
$$

Using the definition of $a_{1}(0)$, we have, in terms of the original model,

$$
\left\langle\mathbf{W}_{1}(\xi) \mid \mathbf{u}_{\mathrm{s}}(\xi+s)\right\rangle=\left\langle\mathbf{W}_{1}(\xi) \mid \hat{\mathbf{u}}(\xi)-\mathbf{u}_{r}\right\rangle .
$$

This is an equation of the center-stable space, i.e. a tangent space to the center-stable manifold of the critical solution. Note that this tangent space is different for every choice of the critical solution as identified by the choice of $s$.

\section{B. Linear approximation of the strength-extent curve}

\section{General setting}

Let us now consider the typical formulation, when the spatial profile of the initial perturbation is fixed and only its magnitude is varied,

$$
\mathbf{u}_{\mathrm{s}}(x)=U_{\mathrm{s}} \mathbf{X}(x) \text {. }
$$


Then (24) gives

$$
U_{\mathrm{s}}\left\langle\mathbf{W}_{1}(\xi) \mid \mathbf{X}(\xi+s)\right\rangle=\left\langle\mathbf{W}_{1}(\xi) \mid \hat{\mathbf{u}}(\xi)-\mathbf{u}_{r}\right\rangle
$$

or

$$
U_{\mathrm{s}}=\frac{\mathcal{N}_{1}}{\mathcal{D}_{1}(s)}
$$

where the numerator $\mathcal{N}_{1}$ is a constant, defined entirely by the properties of the model,

$$
\mathcal{N}_{1}=\left\langle\mathbf{W}_{1}(\xi) \mid \hat{\mathbf{u}}(\xi)-\mathbf{u}_{r}\right\rangle
$$

and the denominator $\mathcal{D}_{1}$ depends on the shift $s$,

$$
\mathcal{D}_{1}(s)=\left\langle\mathbf{W}_{1}(\xi) \mid \mathbf{X}(\xi+s)\right\rangle \text {. }
$$

Hence to get the ultimate answer, we need an extra condition to fix the value of the shift $s$.

\section{The case of critical nucleus}

This is the case when $c=0$, i.e. the critical solution is stationary, and moreover it is even in $x$. Then there is a natural choice of $s=0$ prescribed by symmetry. It can also be motivated directly by considering the problem for $x \in \mathbb{R}$ as an even extension of the problem for $x \in \mathbb{R}_{+}$. In this case the position of the critical nucleus is fixed, there is no translational invariance, no associated zero eigenvalue, and we can consider the stable space, tangent to the stable manifold, as symbolised in fig. 1(d), rather than center-stable manifold.

That is, we have $x=\xi, t=\tau, \mathbf{u}=\tilde{\mathbf{u}}, \hat{\mathbf{u}}(-\xi) \equiv \hat{\mathbf{u}}(\xi)$, and (26) gives the explicit expression for the threshold

$$
U_{\mathrm{s}}=\frac{\int_{0}^{\infty} \mathbf{W}_{1}(\xi)^{\top}\left(\hat{\mathbf{u}}(\xi)-\mathbf{u}_{r}\right) \mathrm{d} \xi}{\int_{0}^{\infty} \mathbf{W}_{1}(\xi)^{\top} \mathbf{X}(\xi) \mathrm{d} \xi} .
$$

If, further, the stimulation is done by a rectangular perturbation of the resting state,

$$
\mathbf{X}(\xi)=\mathrm{H}\left(x_{\mathrm{s}}-\xi\right) \mathrm{H}\left(x_{\mathrm{s}}+\xi\right) \mathbf{e},
$$

then we have

$$
U_{\mathrm{s}}=\frac{\int_{0}^{\infty} \mathbf{W}_{1}(\xi)^{\top}\left(\hat{\mathbf{u}}(\xi)-\mathbf{u}_{r}\right) \mathrm{d} \xi}{\int_{0}^{x_{\mathrm{s}}} \mathbf{W}_{1}(\xi)^{\top} \mathbf{e} \mathrm{d} \xi} .
$$

\section{The case of moving critical solution}

This is the case when $c>0$, and then we call the critical solution either a critical pulse (for $\mathbf{u}_{-}=\mathbf{u}_{r}$ ) or a critical front $\left(\mathbf{u}_{-} \neq \mathbf{u}_{r}\right)$ 27. The problem now does not have

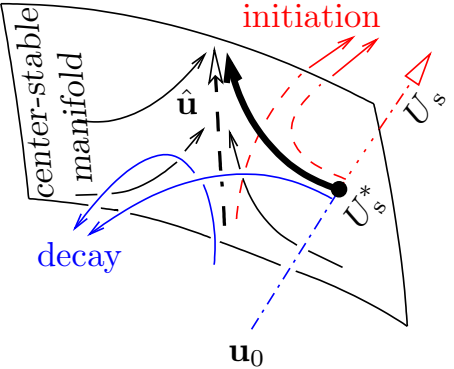

FIG. 2: (Color online) The sketch of a center-stable manifold of a moving critical solution. The critical solution is represented by the dashed bold black line; the critical trajectories, constituting the center-stable manifold, are shown in thin solid black lines. The family of initial conditions is represented by the dash-dotted lines. The bold solid black line is the critical trajectory with initial condition in that family. The sub-threshold trajectories are represented by the thin blue lines, while the thin red lines represent super-threshold trajectories. Note that the point where the initial condition intersect the center-stable manifold is shown as the filled circle.

the symmetry $\xi \mapsto-\xi$ and the previous "intuitively obvious" choice of $s$ is not generally applicable. Recall that our approach is based on linearization, whereas the original problem does not, in fact, contain small parameters. In this formulation, the criticality condition depends on an "arbitrary" parameter $s$. We select an optimal value of the parameter, so as to minimize the error in the prediction. This is done based on a heuristic, motivated by the "skew-product" approach to the dynamics of systems with continuous symmetries, such as in [28] for symmetry with respect to shifts in $\mathbb{R}$ as in our present case, and also in 29, 30 for symmetry with respect to Euclidean motions in $\mathbb{R}^{2}$. This approach considers solutions $\mathbf{u}(x, t)$ of (1) in the form

$$
\mathbf{u}(x, t)=\tilde{\mathbf{u}}(\xi, \tau),
$$

where $\xi=x-s(t), \tau=t$, so $\tilde{\mathbf{u}}(\cdot, \tau)$ describes the evolution of the shape of the wave profile in a frame of reference which moves according to the law defined by the shift $s(t)$, and the dynamics of the shift $s(t)$ is determined from an extra condition, such as

$$
\mu(\mathbf{u}(s(t), t)) \equiv 0,
$$

for an appropriately selected function $\mu(\cdot)$, which allows to choose a unique value of $s$ for any given profile $\mathbf{u}(\cdot, t)$, from the class that is of interest to our study, at any given time $t$, perhaps with some inequalities to distinguish the front from the back. The specific examples of this extra condition, considered in 28 30 included a condition on $\tilde{\mathbf{u}}(\xi, \tau)$ at a selected point $\xi$. A more generic way discussed in 30 is to use any functional $\mu(\cdot)$ on $\tilde{\mathbf{u}}$ which is not invariant with respect to the group of translations of $\xi$, so that the functional could take a certain value only at selected values of $s$, say typically $\partial_{s} \mu(\tilde{\mathbf{u}}(\xi+s, \tau)) \neq 0$ 
and certainly $\left.\partial_{s} \mu(\hat{\mathbf{u}}(\xi+s))\right|_{s=0} \neq 0$. A popular and efficient choice of such functional can be made when one considers perturbations of a relative equilibrium, as done e.g. in 31 33. This choice is based on the following observation, adapted to our case of a one-parametric symmetry group. An infinitesimal increment of the shift $s$ is equivalent, in this situation, to a corresponding infinitesimal change of coefficient $a_{j}$ in an expansion like $(19)$. Let $\mathbf{V}_{j}(\xi)=\hat{\mathbf{u}}^{\prime}(\xi)$ be the "translational" mode, corresponding to $\lambda_{j}=0$. Then a (locally) unique fixation of $s$ can be achieved by requiring that $a_{j}=0$. In our present situation, the index of the projector to the shift mode is $j=2$. The resulting extra requirement is to be applied to the solution at all moments of time, including the initial condition, for which it gives

$$
\left\langle\mathbf{W}_{2}(\xi) \mid \mathbf{u}_{r}+\mathbf{u}_{\mathbf{s}}(\xi+s)-\hat{\mathbf{u}}(\xi)\right\rangle=0,
$$

leading to

$$
U_{\mathrm{s}}\left\langle\mathbf{W}_{2}(\xi) \mid \mathbf{X}(\xi+s)\right\rangle=\left\langle\mathbf{W}_{2}(\xi) \mid \hat{\mathbf{u}}(\xi)-\mathbf{u}_{r}\right\rangle .
$$

Another interpretation of the same requirement is that the condition $a_{2}(0)=0$, in addition to the already imposed condition of criticality $a_{1}(0)=0$, makes sure that at least two first terms in the Fourier series $(19)$ are zero, thus making $\mathbf{v}(\xi, 0)$ "smaller" in that sense.

The two conditions give a system of two similar equations,

$$
\left\{\begin{array}{l}
U_{\mathrm{s}} \mathcal{D}_{1}(s)=\mathcal{N}_{1}, \\
U_{\mathrm{s}} \mathcal{D}_{2}(s)=\mathcal{N}_{2},
\end{array}\right.
$$

where

$$
\mathcal{N}_{\ell}=\left\langle\mathbf{W}_{\ell}(\xi) \mid \hat{\mathbf{u}}(\xi)-\mathbf{u}_{r}\right\rangle, \quad \ell=1,2
$$

and

$$
\mathcal{D}_{\ell}(s)=\left\langle\mathbf{W}_{\ell}(\xi) \mid \mathbf{X}(\xi+s)\right\rangle, \quad \ell=1,2 .
$$

The definitions of $\mathcal{N}_{1}, \mathcal{D}_{1}$ here agree with the ones given earlier in 27, 28). We note that, if $\hat{\mathbf{u}}_{-} \neq \mathbf{u}_{r}$, integrals (35) converge if $\mathbf{W}_{\ell}(\xi \rightarrow-\infty) \rightarrow 0$ sufficiently quickly.

System (34) is a nonlinear system of two equations for two unknowns, $s$ and $U_{\mathrm{s}}$. It is linear and over-determined with respect to $U_{\mathrm{s}}$. The compatibility condition for the two equations for $U_{\mathrm{s}}$ is $\mathcal{N}_{1} \mathcal{D}_{2}(s)-\mathcal{N}_{2} \mathcal{D}_{1}(s)=0$, or

$$
\langle\boldsymbol{\Phi}(\xi) \mid \mathbf{X}(\xi+s)\rangle=0
$$

where

$$
\boldsymbol{\Phi}(\xi)=\mathcal{N}_{1} \mathbf{W}_{2}(\xi)-\mathcal{N}_{2} \mathbf{W}_{1}(\xi),
$$

presenting a nonlinear equation for $s$. For a rectangular stimulus profile,

$$
\mathbf{X}(x)=\mathrm{H}\left(x+x_{\mathrm{s}}\right) \mathrm{H}\left(x_{\mathrm{s}}-x\right) \mathbf{e},
$$

the compatibility condition becomes

$$
\int_{-s-x_{\mathrm{s}}}^{-s+x_{\mathrm{s}}} \Phi(\xi) \mathrm{d} \xi=0
$$

where

$$
\Phi(\xi)=\mathbf{e}^{\top} \boldsymbol{\Phi}(\xi)
$$

This equation for $s$ can be transformed into a more convenient form if we introduce the anti-derivative of $\Phi(\xi)$,

$$
\Phi(\xi)=\eta^{\prime}(\xi) .
$$

Then

$$
\eta\left(-s+x_{\mathrm{s}}\right)-\eta\left(-s-x_{\mathrm{s}}\right)=0,
$$

that is, the two points $\xi^{+}=-s+x_{\mathrm{s}}$ and $\xi^{-}=-s-x_{\mathrm{s}}$ are points of equal value of function $\eta(\cdot)$. If this function happens to be unimodal, then a unique solution of the compatibility condition is guaranteed to exist, and if its monotonic pieces $\eta^{+}(\cdot)$ and $\eta^{-}(\cdot)$ are effectively invertible with, say, $\operatorname{dom}\left(\eta^{+}\right)>\operatorname{dom}\left(\eta^{-}\right)$pointwise, then (39) leads to a parametric equation for the critical curve $\bar{U}_{\mathrm{s}}\left(x_{\mathrm{s}}\right)$. If we denote the value of function $\eta(\cdot)$ in $(39)$ by $\zeta$ and take it as the parameter, then we have

$$
\begin{aligned}
& \xi^{ \pm}(\zeta)=\left(\eta^{ \pm}\right)^{-1}(\zeta), \\
& x_{\mathrm{s}}(\zeta)=\frac{1}{2}\left(\xi^{+}(\zeta)-\xi^{-}(\zeta)\right), \\
& s(\zeta)=-\frac{1}{2}\left(\xi^{+}(\zeta)+\xi^{-}(\zeta)\right), \\
& U_{\mathrm{s}}(\zeta)=\mathcal{N}_{1} / \mathcal{D}_{1}(s(\zeta)) .
\end{aligned}
$$

For reference, we also summarise here the definitions of the ingredients of 40 given earlier:

$$
\begin{aligned}
& \eta(\xi)=\mathcal{N}_{1} \mathcal{I}_{2}(\xi)-\mathcal{N}_{2} \mathcal{I}_{1}(\xi), \\
& \mathcal{I}_{\ell}(\xi)=\int^{\xi} \mathbf{e}^{\top} \mathbf{W}_{\ell}\left(\xi^{\prime}\right) \mathrm{d} \xi^{\prime}, \ell=1,2 \\
& \mathcal{N}_{\ell}=\int_{-\infty}^{\infty} \mathbf{W}_{\ell}^{\top}(\xi)\left(\hat{\mathbf{u}}(\xi)-\mathbf{u}_{r}\right) \mathrm{d} \xi, \ell=1,2 \\
& \mathcal{D}_{1}(s)=\mathcal{I}_{1}\left(\xi^{+}\right)-\mathcal{I}_{1}\left(\xi^{-}\right) .
\end{aligned}
$$

We note that in the case of a critical nucleus, $c=0$, $\hat{\mathbf{u}}$ is an even function, the operators $\mathcal{L}$ and $\mathcal{L}^{+}$commute with the operator of inversion $\xi \mapsto-\xi$, the function $\mathbf{W}_{1}$ is even, the function $\mathbf{W}_{2}$ is odd, $\mathcal{N}_{2}=0, \mathcal{I}_{2}$ is even, $\eta$ is even, $\xi^{+}=-\xi^{-}, s=0$ and 40 formally recovers the result 26) obtained previously based on the choice $s=0$ as "intuitive" and "natural".

\section{Quadratic approximation of the stable manifold}

The use of a linear approximation around the critical solution for the situation when distance from it is not 
guaranteed to be very small is, admittedly, the weakest point of our approach. In this section, we consider the second-order approximation, in order to assess the limits of applicability of the linear approximation, and possibly to improve it. We restrict the consideration to the case of critical nucleus. We use the formulation on $x \in(-\infty, \infty)$ and on the space of even functions $u(\cdot, t)$.

Rather than using the matrix notation as in the linear approximation, we shall now proceed with an explicit notation for the components of the reaction-diffusion systems. We use Greek letters for superscripts to enumerate them, and adopt Einstein's summation convention for those indices. In this way we start from the generic reaction-diffusion system

$$
\frac{\partial u^{\alpha}}{\partial t}=D^{\alpha \beta} \frac{\partial^{2} u^{\beta}}{\partial x^{2}}+f^{\alpha}\left(u^{\beta}\right)
$$

then consider the deviation $v^{\alpha}$ of the solution $u^{\alpha}$ from the critical nucleus $\hat{u}^{\alpha}$,

$$
u^{\alpha}(x, t)=\hat{u}^{\alpha}(x)+v^{\alpha}(x, t),
$$

the equation defining the critical nucleus,

$$
D^{\alpha \beta} \frac{\partial^{2} \hat{u}^{\beta}}{\partial x^{2}}+f^{\alpha}(\hat{\mathbf{u}})=0
$$

and the Taylor expansion of the equation for the deviation,

$$
\dot{v}^{\alpha}=D^{\alpha \beta} v_{x x}^{\beta}+f_{, \beta}^{\alpha}(\hat{\mathbf{u}}) v^{\beta}+\frac{1}{2} f_{, \beta \gamma}^{\alpha}(\hat{\mathbf{u}}) v^{\beta} v^{\gamma}+\ldots,
$$

where overdots denote differentiation with respect to time, subscripts $(\cdot)_{x}$ denote differentiation with respect to space and Greek subscripts after a comma designate a partial differentiation by the corresponding reactive components. The right and left eigenfunctions are defined respectively by

$$
D^{\alpha \beta} \partial_{x x} V_{j}^{\beta}(x)+f_{, \beta}^{\alpha}(x) V_{j}^{\beta}(x)=\lambda_{j} V_{j}^{\alpha}(x)
$$

and

$$
D^{\beta \alpha} \partial_{x x} W_{j}^{\beta}(x)+f_{, \alpha}^{\beta}(x) W_{j}^{\beta}(x)=\lambda_{j} W_{j}^{\alpha}(x),
$$

where $j \in\{1,2,3, \ldots\}$, and the biorthogonality condition is

$$
\left\langle\mathbf{W}_{j} \mid \mathbf{V}_{k}\right\rangle \triangleq \int_{-\infty}^{\infty} \overline{W_{j}^{\alpha}(x)} V_{k}^{\alpha}(x) \mathrm{d} x=\delta_{j, k}
$$

We consider only even solutions, so in subsequent sums only those $j$ that correspond to even eigenfunctions are assumed. We seek solutions in the form of generalized Fourier series in terms of the right eigefunctions,

$$
v^{\alpha}(x, t)=\sum_{j} a_{j}(t) V_{j}^{\alpha}(x)
$$

where the Fourier coefficients are defined by

$$
a_{j}(t)=\left\langle\mathbf{W}_{j}(x) \mid \mathbf{v}(x, t)\right\rangle \triangleq \int_{-\infty}^{\infty} \overline{W_{j}^{\alpha}(x)} v^{\alpha}(x, t) \mathrm{d} x .
$$

Time-differentiation of this gives

$$
\dot{a}_{j}=\lambda_{j} a_{j}+\sum_{m, n} Q_{m, n}^{j} a_{m} a_{n}
$$

where

$$
\begin{aligned}
Q_{m, n}^{j} & =Q_{n, m}^{j} \\
& \triangleq \frac{1}{2} \int_{-\infty}^{\infty} \overline{W_{j}^{\alpha}(x)} f_{, \beta \gamma}^{\alpha}(\hat{\mathbf{u}}(x)) V_{m}^{\beta}(x) V_{n}^{\gamma}(x) \mathrm{d} x .
\end{aligned}
$$

We assume that eigenvalues are real and ordered from larger to smaller, $\lambda_{1}>0, \lambda_{2}=0$ is of course the eigenvalue corresponding to the translational symmetry and an odd eigenfunction $\mathbf{V}_{2}=\hat{\mathbf{u}}^{\prime}$, and $\lambda_{j}<0$ for all $j \geq 3$. Our task is to determine the conditions on the initial values of the Fourier coefficients

$$
A_{j} \triangleq a_{j}(0)=\int_{-\infty}^{\infty} W_{j}^{\alpha}(x) v^{\alpha}(x, 0) \mathrm{d} x
$$

that would ensure that

$$
a_{1}(\infty)=0
$$

which means that the trajectory approaches the critical nucleus, so the initial condition is precisely at the threshold.

Let us rewrite system as an equivalent system of integral equations,

$$
a_{j}(t)=\mathrm{e}^{\lambda_{j} t}\left(A_{j}+\int_{0}^{t} \mathrm{e}^{-\lambda_{j} t^{\prime}} \sum_{m, n} Q_{m, n}^{j} a_{m}\left(t^{\prime}\right) a_{n}\left(t^{\prime}\right) \mathrm{d} t^{\prime}\right) .
$$

Successive approximations to the solution can be obtained by direct iterations of this system,

$$
\begin{gathered}
a_{j}^{(i+1)}(t)= \\
\mathrm{e}^{\lambda_{j} t}\left(A_{j}+\int_{0}^{t} \mathrm{e}^{-\lambda_{j} t^{\prime}} \sum_{m, n} Q_{m, n}^{j} a_{m}^{(i)}\left(t^{\prime}\right) a_{n}^{(i)}\left(t^{\prime}\right) \mathrm{d} t^{\prime}\right) .
\end{gathered}
$$

Taking $a_{j}^{(0)}=0$ for all $j$, we have

$$
a_{j}^{(1)}=A_{j} \mathrm{e}^{\lambda_{j} t} .
$$

With account of $\lambda_{1}>0, \lambda_{j}<0, j \geq 3$, and $a_{j}^{(1)}(t) \rightarrow 0$, this implies that

$$
A_{1}=0, \quad A_{j} \in \mathbb{R}, \quad j \geq 3,
$$


which is the answer we have from the linear approximation. The next iteration produces

$$
\begin{aligned}
a_{j}^{(2)}(t)= & \mathrm{e}^{\lambda_{j} t}\left(A_{j}+\sum_{m, n \geq 3} \frac{Q_{m, n}^{j} A_{m} A_{n}}{\lambda_{j}-\lambda_{m}-\lambda_{n}}\right) \\
& -\sum_{m, n \geq 3} \frac{Q_{m, n}^{j} A_{m} A_{n}}{\lambda_{j}-\lambda_{m}-\lambda_{n}} \mathrm{e}^{\left(\lambda_{m}+\lambda_{n}\right) t} .
\end{aligned}
$$

Assuming that the sums converge, the last term always tends to zero as $t \rightarrow \infty$ because $\lambda_{n} \leq \lambda_{3}<0$ for all $n \geq 3$, and the first term tends to zero for all $j \geq 3$ for the same reason. So, the condition $a_{1}^{(2)}(t) \rightarrow 0$ implies that that the first term vanishes for $j=1$, that is,

$$
A_{1}=-\sum_{m, n \geq 3} \frac{Q_{m, n}^{1} A_{m} A_{n}}{\lambda_{1}-\lambda_{m}-\lambda_{n}}
$$

which is our second-order (quadratic) approximation for the critical condition, as opposed to the first-order (linear) approximation which states simply that $A_{1}=0$. We see that the linear approximation will be more accurate when $A_{n}, n \geq 3$ are smaller, and that for given magnitudes of $A_{n}$, the linear approximation will be better if $\lambda_{1}-\lambda_{m}-\lambda_{n}$, the smallest of which is $\lambda_{1}-2 \lambda_{3}$, is larger (remember we exclude all eigenpairs with odd eigenfunctions, including $n=2$ ).

Further iterations of (47) lead to still higher-order approximations of the stable manifold of the critical nucleus, and possibly further improvement of the critical condition. This, however, is beyond the scope of this paper.

Substitution into 48 of the definition of $A_{j}$ in terms of the stimulation amplitude,

$$
A_{j}=\int_{-\infty}^{\infty} \mathbf{W}_{j}(x)^{\top}\left(\mathbf{u}_{r}-\hat{\mathbf{u}}(x)+U_{\mathrm{s}} \mathbf{X}(x)\right) \mathrm{d} x
$$

leads to a quadratic equation for $U_{\mathrm{s}}$,

$$
A U_{\mathrm{s}}^{2}+B U_{\mathrm{s}}+C=0
$$

where

$$
\begin{aligned}
A & =\sum_{n, m \geq 3} R_{m, n} \mathcal{D}_{m} \mathcal{D}_{n} \\
B & =\mathcal{D}_{1}-2 \sum_{n, m \geq 3} R_{m, n} \mathcal{N}_{m} \mathcal{D}_{n} \\
C & =-\mathcal{N}_{1}+\sum_{n, m \geq 3} R_{m, n} \mathcal{N}_{m} \mathcal{N}_{n}
\end{aligned}
$$

and

$$
\begin{aligned}
& R_{m, n}=\frac{Q_{m, n}^{1}}{\lambda_{1}-\lambda_{m}-\lambda_{n}}=R_{n, m}, \\
& \mathcal{N}_{j}=\int_{-\infty}^{\infty} \mathbf{W}_{j}(x)^{\top}\left(\hat{\mathbf{u}}-\mathbf{u}_{r}\right) \mathrm{d} x, \\
& \mathcal{D}_{j}=\int_{-\infty}^{\infty} \mathbf{W}_{j}(x)^{\top} \mathbf{X}(x) \mathrm{d} x .
\end{aligned}
$$

Note that the definitions of $\mathcal{N}_{j}, \mathcal{D}_{j}$ here are the same as in (35), 36), with account of $s=0$.

An essential detail is the question of the properties of the spectra of $\mathcal{L}$ and $\mathcal{L}^{+}$. In the above derivation we assumed that these two spectra coincide, are discrete and all eigenvalues are simple. In the specific cases we consider below, these assumptions will be tested numerically; in particular, we shall observe that the spectra can in fact be continuous, so the formulas should be generalized, to replace summation over eigenvalues by integrals with respect to the spectral measure, and the convergence issue becomes even more complicated.

\section{Hybrid approach: numerical computation of functions required by the analytical theory}

\section{Rationale}

The key to our linear approximation is the knowledge of $\hat{\mathbf{u}}(x), \mathbf{W}_{1}(x)$ and, for thenon-self-adjoint cases, also of $\mathbf{W}_{2}(x)$. For the quadratic approximation, ideally the whole spectrum of $\lambda_{\ell}, \mathbf{W}_{\ell}, \mathbf{V}_{\ell}$ is needed. With a few fortunate exceptions, some of which will be discussed below, one does not have these analytically, so in practically interesting cases, one would need to employ a hybrid approach, where these key ingredients are determined numerically before the analytical expressions (31) or 40 can be applied. The numerical problems can be posed as rather standard boundary-value problems, respectively nonlinear, for $\hat{\mathbf{u}}$, and linear, for $\lambda_{\ell}, \mathbf{W}_{\ell}, \mathbf{V}_{\ell}$. Here we describe the methods we used in specific examples presented later.

In all cases, for direct numerical simulation of timedependent problems, we discretize the problems on a regular space grid on a finite interval $x \in[0, L]$ as an approximation of $x \in[0, \infty)$, with fixed space step $\Delta_{x}$ and a regular time grid with time step $\Delta_{t}$. Except where stated otherwise, we use second-order central difference approximations in space, with Neumann boundary conditions at $x=L$, and explicit first-order forward Euler method in time. 


\section{The case of critical nucleus}

a. Shooting Finding $\hat{\mathbf{u}}$ means solving a nonlinear boundary-value problem. Most of the advanced methods require a good initial guess for the solution. We find this initial guess by a version of the shooting method. We solve a sequence of the "stimulation by voltage" initialvalue problem (56), with fixed stimulation extent $x_{\mathrm{s}}$ and varying amplitude $U_{\mathrm{s}}$. The sequence is selected with the goal of approaching the threshold value for $U_{\mathrm{s}}$ which we denote as $U_{\mathrm{s}}^{*}$. This is done using the bisection method. Starting from an upper estimate $\overline{U_{\mathrm{s}}}$, known to be sufficient for ignition, and a lower estimate $U_{\mathrm{s}}$, known to fail to ignite, we proceed with the following algorithm:

\section{repeat}

$$
U_{\mathrm{s}}^{\#}:=\frac{1}{2}\left(\overline{U_{\mathrm{s}}}+\underline{U_{\mathrm{s}}}\right) \text { (the new trial value of } U_{\mathrm{s}} \text { is }
$$

the average of the current upper and lower estimates of the threshold);

Solve the initial value problem with $U_{\mathrm{s}}=U_{\mathrm{s}}{ }^{\#}$ and determine if ignition or failure;

\section{if ignition then}

$\overline{U_{\mathrm{s}}}:=U_{\mathrm{s}} \#$ (the trial value of $U_{\mathrm{s}}$ will become the new upper estimate for the threshold);

else

$U_{\mathrm{s}}:=U_{\mathrm{s}}{ }^{\#}$ (the trial value of $U_{\mathrm{s}}$ will become the new lower estimate for the threshold); end if

until $\left(\left|\overline{U_{\mathrm{s}}}-\underline{U_{\mathrm{s}}}\right| \leq\right.$ tolerance $)$

In fact, to achieve the best result, we typically use zero tolerance, i.e. repeat the bisection loop as long as $U_{\mathrm{s}} \#$ remains distinct from both $\overline{U_{\mathrm{s}}}$ and $U_{\mathrm{s}}$ given the machine epsilon. The final value of $U_{\mathrm{s}} \#$ is the approximation of the critical amplitude $U_{\mathrm{s}}^{*}$ for the given $x_{\mathrm{s}}$, the best achievable one at a given discretization. More precisely, we consider the last values of $\overline{U_{\mathrm{s}}}$ and $U_{\mathrm{s}}$ as equally likely approximations $U_{\mathrm{s}} \#$ of $U_{\mathrm{s}}^{*}$, as which of them happens to be equal to $\left(\overline{U_{\mathrm{s}}}+U_{\mathrm{s}}\right) / 2$ in computer arithmetics is determined only by their position in the grid of floatingpoint numbers in the given architecture, rather than their relative merits as approximations.

The so found estimate of $U_{\mathrm{s}}^{*}\left(x_{\mathrm{s}}\right)$ is used to determine an estimate for $\hat{\mathbf{u}}$. The idea is based on the observation that for $U_{\mathrm{s}}$ very close to the exact threshold $U_{\mathrm{s}}^{*}\left(x_{\mathrm{s}}\right)$, the trajectory approaches the saddle point $\hat{\mathbf{u}}(x)$ to within a small distance and remains in its vicinity for a long time, see fig. 1(d). So, to find the best estimate for the threshold trajectory obtained for $U_{\mathrm{s}}=U_{\mathrm{s}}{ }^{\#}$, we calculate

$$
S(t)=\|\dot{\mathbf{u}}\|_{L^{2}}^{2}=\int_{0}^{L}\left\|\mathbf{u}_{t}\right\|^{2}(x, t) \mathrm{d} x
$$

along the trajectory, find $t^{\#}=\operatorname{argmin}(S(t))$ and take $\hat{\mathbf{u}}^{\#}(x)=\mathbf{u}\left(x, t^{\#}\right)$ as an estimate of the critical nucleus $\hat{\mathbf{u}}(x)$. The result can be immediately used for the next step or serve as an initial guess for a more advanced boundary-value solver if a higher accuracy is required.
Note that the key assumption of our theory is that the threshold manifold is the center manifold of a unique critical nucleus solution, hence the above procedure should produce (nearly) the same $\hat{\mathbf{u}}(x)$ from any choice of $x_{\mathrm{s}}$. We used the procedure for different values of $x_{\mathrm{s}}$, both to verify the validity of this key assumption, and to assess the accuracy of the found critical nucleus.

b. Marching The so found approximation of the critical nucleus $\hat{\mathbf{u}}^{\#}(x)$ is then used to calculate the principal eigenvalue $\lambda_{1}$ and the corresponding eigenfunction $\mathbf{W}_{1}$. Since $\lambda_{1}$ is by definition the eigenvalue with the largest real part, we should expect that the solution of the differential equation

$$
\frac{\partial \mathbf{w}}{\partial t}=\mathcal{L}^{+} \mathbf{w} \triangleq \mathbf{D}^{\top} \frac{\partial^{2} \mathbf{w}}{\partial x^{2}}+\mathbf{F}^{\top}(x) \mathbf{w}
$$

for almost any initial conditions, should satisfy

$$
\mathbf{w}(x, t)=C \mathrm{e}^{\lambda_{1} t} \mathbf{W}_{1}(x)(1+o(1)), \quad t \rightarrow \infty .
$$

for some constant $C$. We therefore consider the graph of $\ln |\mathbf{w}(0, \tau)|$, determine the linear part in it, and fit that linear part to a straight line by least squares; the slope provides an estimate of $\lambda_{1}$. We have also verified that the profile $\mathbf{w}(x, t) / \mathbf{w}(0, t)$ remains virtually unchanged during this linear part, and took the most recent profile as $\mathbf{W}_{1}(x)$. Operationally, this is practically equivalent to the (more usual) procedure of estimating the eigenvalue $\lambda_{1}$ for a time interval from $t$ to $t+\delta t$ as $\delta t^{-1} \ln (\langle\mathbf{w}(x, t+\delta t) \mid \mathbf{w}(x, t)\rangle /\langle\mathbf{w}(x, t) \mid \mathbf{w}(x, t)\rangle)$ and then ensuring that this estimate converges as $t \rightarrow \infty$. Again, thus obtained $\lambda_{1}$ and $\mathbf{W}_{1}(x)$ can be immediately used or serve as an initial approximation for a more sophisticated eigenvalue problem solver if a better accuracy is required.

This method is of course easily extended to calculate not just the principal eigenpair, but a number of eigenpairs with largest eigenvalues as long as they are real. If $\mathcal{L}=\mathcal{L}^{+}$then one only needs to calculate $\sqrt{53}$ for a number of linearly independent initial conditions, and at each step, in addition to normalization, also perform the Gram-Schmidt procedure. As discussed above, normalization of the first of the linearly independent solutions gives approximations of $\mathbf{W}_{1}$ and $\lambda_{1}$. The second linearly independent solution is used to obtain a solution orthogonal to $\mathbf{W}_{1}$, which provides an approximation for $\mathbf{W}_{2}$, and its normaliztion provides and approximation for $\lambda_{2}$ from its normalization. Then the third linearly independent solution is used to obtain a solution orthogonal both to $\mathbf{W}_{1}$ and $\mathbf{W}_{2}$, which gives an approximation for $\mathbf{W}_{3}$, and the corresponding normalization gives $\lambda_{3}$, and so on. In the non-self-adjoint case, $\mathcal{L} \neq \mathcal{L}^{+}$, the orthogonalization will of course require this procedure to be done both for $\mathcal{L}$ and $\mathcal{L}^{+}$hand in hand, to calculate $\mathbf{V}_{2}$ as orthogonal to $\mathbf{W}_{1}$ and $\mathbf{W}_{2}$ as orthogonal to $\mathbf{V}_{1}$ and so on. 


\section{The case of moving critical solution}

a. Co-moving frame of reference We use the idea of symmetry reduction employed in Section II B 3 for the theory, now for numerical simulations to reduce the problem of moving critical solution to the case of a stationary critical solution, even though with non-self-adjoint linearization. To this end, we consider the equation

$$
\frac{\partial \mathbf{u}}{\partial t}=\mathbf{D} \frac{\partial^{2} \mathbf{u}}{\partial x^{2}}+\mathbf{f}(\mathbf{u})
$$

where the position of the front, $s$, is defined implicitly by

$$
\mu(\mathbf{u}(s, t))=0
$$

and perhaps some extra inequalities to distinguish the front from the back. Then in the comoving frame of reference $\xi=x-s(t), \tau=t$, we have an unknown function of time and space,

$$
\tilde{\mathbf{u}}(\xi, \tau)=\mathbf{u}(x, t),
$$

and an unknown function of time, $s(t)$, the system of PDEs and a finite constraint

$$
\begin{aligned}
& \frac{\partial \tilde{\mathbf{u}}}{\partial \tau}=\mathbf{D} \frac{\partial^{2} \tilde{\mathbf{u}}}{\partial \xi^{2}}+\frac{\mathrm{d} s}{\mathrm{~d} \tau} \frac{\partial \tilde{\mathbf{u}}}{\partial \xi}+\mathbf{f}(\tilde{\mathbf{u}}), \\
& \mu(\tilde{\mathbf{u}}(0, \tau))=0 .
\end{aligned}
$$

A relative equilibrium, including the moving critical solution, in the system (55), corresponds to an equilibrium in (56), so it is possible to use the same techniques developed for the case of a stationary critical solution, to the comoving system (56).

b. Shooting To find the critical solutions, we solve initial value problems for (56) and adjust the initial conditions so as to get as close to the initiation threshold as possible given the machine error. Solutions of (56) were found by semi-implicit time-stepping with the simplest (Lie) operator splitting, with four substeps, namely

1. updating $\tilde{\mathbf{u}}$ by an explicit first-order (forward Euler) scheme, for the nonlinear kinetics term $\mathbf{f}(\tilde{\mathbf{u}})$;

2. updating $\tilde{\mathbf{u}}$ by a semi-implicit (Crank-Nicholson) scheme in time, and central-difference in space, for the diffusion term $\mathbf{D} \frac{\partial^{2} \tilde{\mathbf{u}}}{\partial \xi^{2}}$;

3. finding the convection speed $\frac{\mathrm{d} s}{\mathrm{~d} \tau}$ based on a "virtual" or "predictor" convection substep, that would update $\tilde{\mathbf{u}}$ by an explicit in time, two-point upwind scheme without smoothing;

4. the actual updating of $\tilde{\mathbf{u}}$ by an implicit in time, 3-point upwind scheme with smoothing (BeamWarming, [34]) for the convection term $\frac{\mathrm{d} s}{\mathrm{~d} \tau} \frac{\partial \tilde{\mathbf{u}}}{\partial \xi}$, using the value of $\frac{\mathrm{d} s}{\mathrm{~d} \tau}$ found in the previous substep.
As in the case of critical nucleus, the critical solution is estimated as the slowest point of the trajectory, i.e. at the moment $\tau=\tau^{\#}=\operatorname{argmin}\left\|\tilde{\mathbf{u}}_{\tau}\right\|_{L^{2}}$. This includes $\operatorname{both} \hat{\mathbf{u}}^{\#}(\xi)=\tilde{\mathbf{u}}\left(\xi, \tau^{\#}\right)$ and $c^{\#}=\frac{\mathrm{d} s}{\mathrm{~d} \tau}\left(\tau^{\#}\right)$.

c. Continuation For the examples with nonstationary critical solutions, the accuracy of the critical solution found by shooting was often insufficient and we also found it as a solution of a boundary-value problem (12), which, in dynamical systems terms, is a problem of finding homoclinic (if $\mathbf{u}_{-}=\mathbf{u}_{r}$ ) or heteroclinic (if $\mathbf{u}_{-} \neq \mathbf{u}_{r}$ ) trajectories in a one-parametric family of autonomous systems for $\hat{\mathbf{u}}(\xi)$, with parameter $c$. In the examples presented in this paper, we looked for homoclinics and used a simple and popular continuation method of finding such orbits, as a large-period limits of periodic trajectories of the same system, that is,

$$
\begin{aligned}
& \mathbf{0}=\mathbf{D} \frac{\mathrm{d}^{2} \mathbf{u}_{P}}{\mathrm{~d} \xi^{2}}+c_{P} \frac{\mathrm{d} \mathbf{u}_{P}}{\mathrm{~d} \xi}+\mathbf{f}\left(\mathbf{u}_{P}\right), \\
& \mathbf{u}_{P}(\xi+P) \equiv \mathbf{u}_{P}(\xi),
\end{aligned}
$$

using the continuation software AUTO 35. When the problem is well posed, this defines a curve in the $\left(P, c_{P}, \mathbf{u}_{P}(\xi)\right)$ space. In our examples, the two ends of this curve extend to the limit $P \rightarrow \infty$; one of the ends defining the stable propagating pulse solution, $\left(c_{\mathrm{w}}, \mathbf{u}_{\mathrm{w}}(\xi)\right)$, and the other defining the critical pulse solution, $(c, \hat{\mathbf{u}}(\xi))$, which is of interest to us. An initial guess for the continuation procedure could be obtained from the shooting procedure described above, which would give the initial guess at the unstable branch of the curve, or just by direct numerical simulations of (1) with $P$ periodic boundary conditions in $x$, which would give an initial guess at the stable branch of the curve. However, we preferred to use an ad hoc method, which is very popular for excitable systems, by finding the periodic orbits via Hopf bifurcation in a one-parametric extension of (57), with an extra parameter corresponding to a "stimulation current", that is an additive constant in the equation representing the dynamics of the activator, say the transmembrane voltage.

AUTO uses collocation to discretize the solutions, and subsequent steps in our approach, such as marching and Arnoldi iterations, use $\hat{\mathbf{u}}$ discretized on a regular grid. To interpolate the solution obtained by AUTO to the regular grid, we use piecewise Hermite interpolation [36.

d. Marching Once the critical solution $\hat{\mathbf{u}}^{\#}(\xi)$ and its speed $c^{\#}$ have been found, determination of the right and left eigenfunctions is done by calculating solutions of the initial value problems

$$
\frac{\partial \mathbf{v}}{\partial \tau}=\mathcal{L} \mathbf{v} \triangleq \mathbf{D} \frac{\partial^{2} \mathbf{v}}{\partial \xi^{2}}+c^{\#} \frac{\partial \mathbf{v}}{\partial \xi}+\mathbf{F}(\xi) \mathbf{v}
$$

and

$$
\frac{\partial \mathbf{w}}{\partial \tau}=\mathcal{L}^{+} \mathbf{w} \triangleq \mathbf{D}^{\top} \frac{\partial^{2} \mathbf{w}}{\partial \xi^{2}}-c^{\#} \frac{\partial \mathbf{w}}{\partial \xi}+\mathbf{F}^{\top}(\xi) \mathbf{w} .
$$


The leading eigenvalue $\lambda_{1}$ and the corresponding right eigenfunctions $\mathbf{V}_{1}$ and left eigenfunctions $\mathbf{W}_{1}$ are obtained in the limit $\tau \rightarrow \infty$ for almost any initial conditions in (58) and (59). The second eigenvalue $\lambda_{2}$ and the corresponding eigenfunctions $\mathbf{V}_{2}$ and $\mathbf{W}_{2}$ are obtained as linearly independent solutions of the same equations, satisfying the constraints

$$
\left\langle\mathbf{W}_{2} \mid \mathbf{V}_{1}\right\rangle=\left\langle\mathbf{W}_{1} \mid \mathbf{V}_{2}\right\rangle=0
$$

using a Gram-Schmidt orthogonalization process, adapted to our non-self-adjoint situation.

e. Arnoldi iterations When computing the required eigenvalues and eigenfunctions with the required accuracy took too much time by the marching method discussed above, we used the standard implicitly restarted Arnoldi iterations, using the implementation described in [37. This was applied to the matrices representing the right-hand sides of the discretized versions of equations (59) and (58) to find left and right eigenfunctions respectively. We requested finding eigenvalues with biggest real parts and used the default values of the tuning parameters.

\section{E. A priori bound in the critical nucleus case}

Finally we comment on a simple a priori bound for the critical curve, which follows from considerations different from the analysis of the central-stable manifold of the critical solution, and therefore may provide useful extra information. It applies for the case of $d=1$, when the critical solution is the critical nucleus defined by

$$
\frac{\partial u}{\partial t}=\frac{\partial^{2} u}{\partial x^{2}}+f(u)
$$

with the assumptions that $f\left(u_{j}\right)=0, j=1,2,3, u_{1}=$ $u_{r}<u_{2}<u_{3}, f(u)<0$ for $u \in\left(u_{1}, u_{2}\right)$ and $f(u)>0$ for $u \in\left(u_{2}, u_{3}\right)$. In these terms, successful initiation means that at large $t$ solution $u(x, t)$ is a trigger wave from $u_{1}$ to $u_{3}$, and the failure of initiation means that $u(x, t) \rightarrow u_{1}$ as $t \rightarrow \infty$ uniformly in $x$.

It follows from the results by Fife and McLeod 38, that any initial conditions such that $u(x, 0) \in\left[u_{2}, u_{3}\right]$ for $x \in\left(-\infty, x_{1}\right)$ and $u(x, 0) \in\left[u_{1}, u_{2}\right]$ for $x \in\left(x_{2}, \infty\right)$ guarantee ignition, and for rectangular initial conditions (25) this means that even for the smallest excess of $U_{\mathrm{s}}$ over $u_{2}-u_{1}$, this initial condition will produce ignition, provided that $x_{\mathrm{s}}$ is large enough, so we have

$$
U_{\mathrm{s}}^{*}\left(x_{\mathrm{s}}\right) \searrow \underline{U_{\mathrm{s}}^{*}}, \quad x_{\mathrm{s}} \rightarrow \infty,
$$

where

$$
\underline{U_{\mathrm{s}}^{*}}=u_{2}-u_{1}
$$

In the following sections we verify this general methodology by applying it to five examples.

\section{ZELDOVICH - FRANK-KAMENETSKY EQUATION}

\section{A. Model formulation}

Our first example is the one-component reactiondiffusion equation, first introduced by Zeldovich and Frank-Kamenetsky (ZFK) 39] to describe propagation of flames; it is also known as "Nagumo equation" [40] and "Schlögl model" 41:

$$
\begin{aligned}
& d=1, \quad \mathbf{D}=(1), \quad \mathbf{u}=(u), \\
& \mathbf{f}(\mathbf{u})=(f(u)), \quad f(u)=u(u-\theta)(1-u),
\end{aligned}
$$

where we assume that $\theta \in(0,1 / 2)$.

The critical nucleus solution $\hat{\mathbf{u}}=(\hat{u})$ for this equation can be found analytically [21, 25, 58

$$
\hat{u}(x)=\frac{3 \theta \sqrt{2}}{(1+\theta) \sqrt{2}+\cosh (x \sqrt{\theta}) \sqrt{2-5 \theta+2 \theta^{2}}} .
$$

The other two components required for the definition of critical curves in the linear approximation are $\lambda_{1}$ and $\mathbf{W}_{1}=\mathbf{V}_{1}=\left(V_{1}\right)$ which are solutions of

$$
\begin{aligned}
& \frac{\mathrm{d}^{2} V_{1}}{\mathrm{~d} x^{2}}+\left(-3 \hat{u}^{2}+2(\theta+1) \hat{u}-\theta\right) V_{1}=\lambda_{1} V_{1}, \\
& \lambda_{1}>0, \quad V_{1}( \pm \infty)=0 .
\end{aligned}
$$

We have been unable to find solution of this eigenvalue problem analytically. We note, however, that $\hat{u}$ given by (65) is unimodal, therefore $\hat{u}^{\prime}$, which is the eigenfunction of $\mathcal{L}$ corresponding to $\lambda=0$, has one root, hence by Sturm's oscillation theorem, $\hat{u}^{\prime}=V_{2}$ and $\lambda_{2}=0$, and there is indeed exactly one simple eigenvalue $\lambda_{1}>0$ and the corresponding $V_{1}$ solving 66 has no roots.

\section{B. The small-threshold limit and the "fully analytical" result}

In this subsection we extend the results of [25] in the parameter space and correct some typos found in the latter paper. For $\theta \ll 1$, the critical nucleus $(65)$ is $\mathcal{O}(\theta)$ uniformly in $x$, and is approximately

$$
\hat{u}(x) \approx \frac{3 \theta}{1+\cosh (x \sqrt{\theta})}=\frac{3}{2} \theta \operatorname{sech}^{2}(x \sqrt{\theta} / 2) .
$$

In the same limit, the nonlinearity can be approximated by $f(u) \approx u(u-\theta)$. With these approximations, problem (66) has the solution

$$
\lambda_{1} \approx \frac{5}{4} \theta, \quad V_{1} \approx \operatorname{sech}^{3}(x \sqrt{\theta} / 2),
$$

and (31) then gives an explicit expression for the strength-extent curve in the form

$$
U_{\mathrm{s}}^{*} \approx \frac{9 \pi \theta}{8\left[4 \arctan \left(\mathrm{e}^{\tilde{x_{\mathrm{s}}}}\right)+2 \tanh \left(\tilde{x_{\mathrm{s}}}\right) \operatorname{sech}\left(\tilde{x_{\mathrm{s}}}\right)-\pi\right]}
$$




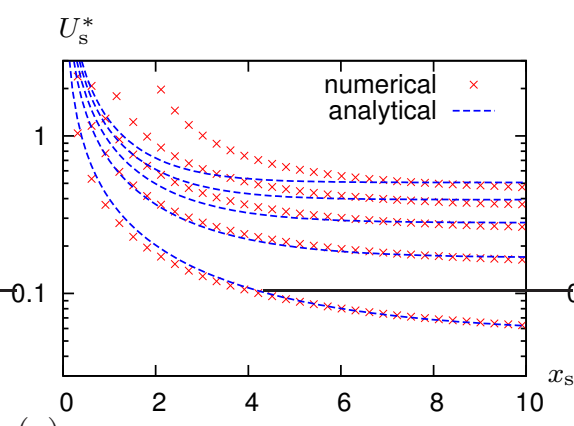

(a)

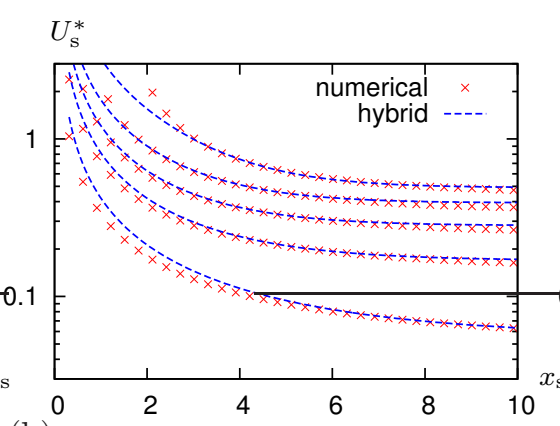

(b)

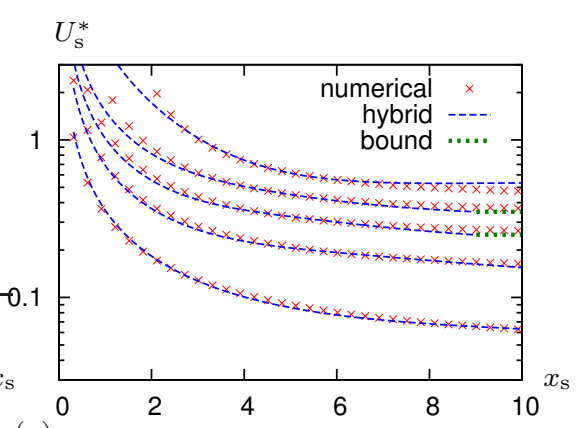

(c)

FIG. 3: (Color online) Strength-extent curves for the ZFK model, for $\theta=0.05,0.15,0.25,0.35,0.45$ (bottom to top), comparison of direct numerical simulations (symbols) with theoretical predictions (dashed lines), (a) for the exact analytical answers in the $\theta \ll 1$ limit, linear approximation; (b) for the hybrid method, using the numerically found ignition eigenpairs, linear approximation; (c) same, quadratic approximation $\sqrt{70}$. Discretization parameters: $\Delta_{x}=0.03, \Delta_{t}=4 \Delta_{x}{ }^{2} / 9, L=100$.

where $\tilde{x_{\mathrm{s}}}=\frac{1}{2} x_{\mathrm{s}} \sqrt{\theta}$. This approximation remains above the a priori lower bound $63, \underline{U}_{\mathrm{s}}^{*}=\theta$, for all $x_{\mathrm{s}}$.

Comparison of this approximation with the direct numerical simulations is shown in fig. 3(a). We see that whereas for small $\theta$ the comparison is reasonable for a wide range of $x_{\mathrm{s}}$, it quickly deteriorates at larger values of $\theta$, which is of course to be expected as the analytical expressions used are only valid in the limit of small $\theta$.

\section{Hybrid approach}

Fig. 4 illustrates the processes of the numerical computation of the critical nucleus (a) and the ignition mode (b) in the ZFK model using the "shooting" algorithm described in Section IID2 for a selected value of the parameter $\theta$. In fig. $4($ a), the minimum of $S(t)$ at about $10^{-5}$, achieved at about $t^{\#} \approx 50$, designates the maximal proximity of the solution $u\left(x, t^{\#}\right)$ of the nonlinear problem (1) to the critical nucleus $\hat{u}(x)$, and so the former can be taken as an approximation of the latter. In fig. 4(b), the solution of the linear problem (53), after an initial transient, mostly expiring before $t=10$, grows exponentially. The increment of this exponential growth gives the ignition eigenvalue $\lambda_{1}$, and the corresponding solution profile, $\mathbf{w}(x, t) / \mathbf{w}(0, t)$, which remains almost unchanged after $t=10$, gives the ignition mode $V_{1}(x)=W_{1}(x)$.

The results of these numerical procedures are shown in fig. 5. We can see that the shooting procedure produces good approximation of the critical nucleus, which for this case is known exactly, for all $\theta$. We also see that the accuracy of the approximation obtained for $\theta \ll 1$, unsurprisingly, is not good for larger $\theta$, see fig. 5(a). The solution of the adjoint problem shown in fig. 5(b,c) demonstrates a nontrivial behaviour qualitatively different from the $\theta \ll 1$ analytical formulas: the eigenvalue starts deviating noticeably from 68 already for $\theta \approx 0.1$, and as $\theta$ continues to increase across approximately 0.3 , a qualitative change happens: the ignition eigenvalue $\lambda_{1}(\theta)$ stops increasing and starts decreasing, and the ignition mode $V_{1}$ stops shrinking and starts expanding, and later even loses the unimodal shape and becomes bimodal, note the $\theta=0.45$ curve in panel (b). The latter property should of course be expected: in the $\theta \nearrow 1 / 2$ limit, the critical nucleus takes the form of two opposite looking fronts separated by the distance $\propto \ln (1 / 2-\theta)$, and the ignition mode is correspondingly a superposition of two sub-modes, each corresponding to its corresponding front, with the ignition eigenvalue $\lambda \searrow 0$.

One more observation can be made in fig. 5(c) about the behaviour of $\lambda_{j}, j>1$. We see that the main assumptions of the theory are satisfied and all these eigenvalues are negative, and moreover, they become more negative for larger $\theta$. Further, the distance $\left|\lambda_{3}-\lambda_{5}\right|$ grows with $\theta$, while a distance $\left|\lambda_{5}-\lambda_{7}\right|$ remains approximately the same and relatively small. This suggests that $\lambda_{3}$ is a point of discrete spectrum, while $\lambda_{5}$ and $\lambda_{7}$ in fact represent already the continuous spectrum and appear as discrete eigenvalues only due to the finite length $L$ of the computational interval. This observation is confirmed by further study of these eigenvalues and the corresponding eigenfunctions: at increasing values of $L$, the distance $\left|\lambda_{5}-\lambda_{7}\right|$ decreases, $V_{1}$ and $V_{3}$ appear well localized towards the left end of the interval $x \in[0, L]$, whereas $V_{5}$ and $V_{7}$ are manifestly non-localized, i.e. vary significantly throughout $x \in[0, L]$ (not shown).

Comparison of the resulting hybrid numericasymptotic prediction with the direct numerical simulations is shown in fig. 3(b). We see that for each value of $\theta$, the reasonable correspondence is observed in some range of $x_{\mathrm{s}}$. The large deviations are observed whenever $U_{\mathrm{s}}^{*}$ gets large, which is expectable since the theory involves a linear approximation, and for large $U_{\mathrm{s}}^{*}$ all $A_{j}$ are large. We also note that for $U_{\mathrm{s}}^{*} \lesssim 1$, the quality of the hybrid approximation is in fact better for larger $\theta$. This is also fully expectable based on the crudest prediction of the quadratic theory: indeed one can see from fig. 5 (c) that the spectral gap $\lambda_{1}-2 \lambda_{3}$, which is related to the accuracy of the linear approximation, grows with $\theta$ (recall the discussion after equation (48)). 
FIG. 4: (Color online) Illustration of the numerical computation of the critical nucleus and ignition mode by "shooting" and "marching" in ZFK. (a) Typical functions $S(t)$ at near-threshold initial conditions in (1), (4), (5), 66), 64). Parameters: $\theta=0.15, x_{\mathrm{s}}=0.6$, $U_{\mathrm{s}}^{*} \approx 1.1676 \ldots,\left|\overline{U_{\mathrm{s}}^{*}}-U_{\mathrm{s}}^{*}\right|<10^{-5}, L=20$, $\Delta_{x}=0.02, \Delta_{t}=4 \Delta_{x}^{2} / 9$. (b) Growth of the numerical solution of 53 in semilogarithmic coordinates, and its linear fit, defining the numerical value of $\lambda_{1} \approx 0.1425$.

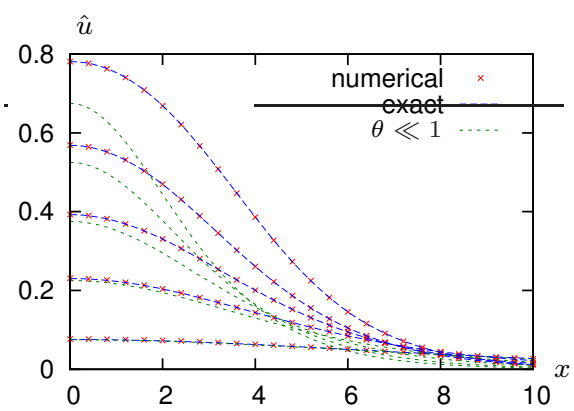

(a)

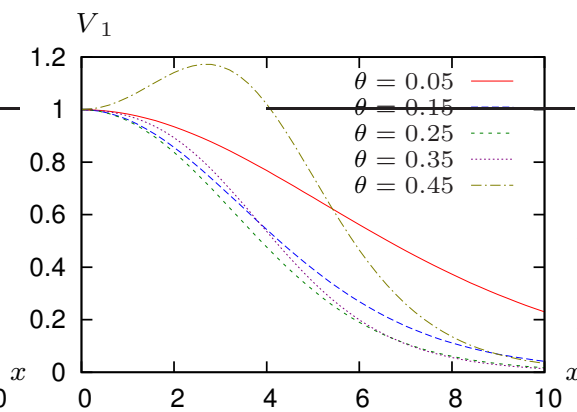

(b) $\ln |\mathbf{w}(0, t)|$

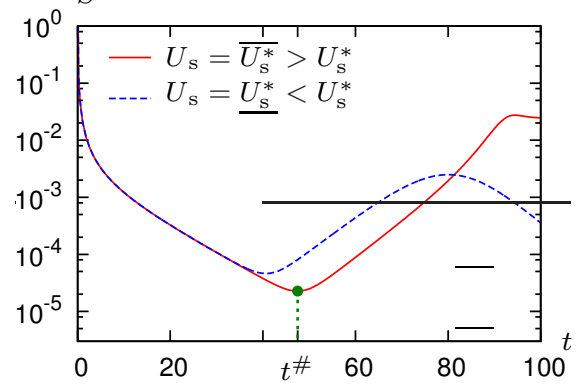

(a)

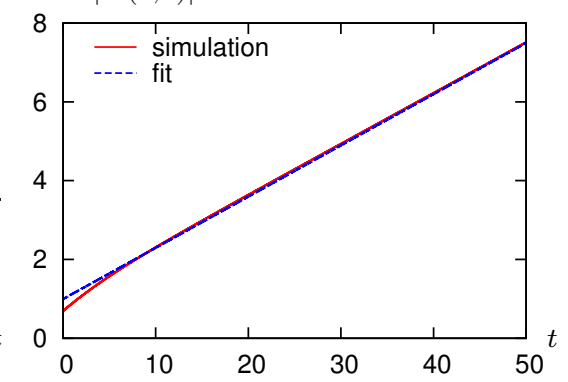

(b)

FIG. 5: (Color online) Numerical computation of the components of the hybrid approach in ZFK. (a) Critical nucleus solutions for a $\theta$ from 0.05 (bottom) to 0.45 (top) with step 0.1 : numerical found by shooting, $\hat{u}^{\#}(x)$; exact analytical given by 65 ; approximate analytical for $\theta \ll 1$ given by (67). (b) The ignition mode, for a selection of values of $\theta$, found by time marching based on numerical critical nucleus. (c) First four eigenvalues, found by marching based on numerical nucleus as functions of $\theta$.

\section{Quadratic theory}

The quadratic theory result given by 49 involves double infinite sums over the stable modes of the linearized problems, so a practical application of this result in its fullness is problematic. However, we note that apart from the generalized Fourier coefficients of the critical nucleus, stimulus profile and the nonlinearity, this expression also has denominators increasing with the stable mode indices, so one may expect that depending on the properties of the spectrum, the terms in the series may quickly decay and one can get a sufficiently accurate result by retaining only a few principal terms. As discussed in the previous subsection, for the ZFK equation, the linearized problem has one discrete stable eigenvalue and the rest of the stable spectrum is continuous. If we retain in 49 ) only the leading term, corresponding to the discrete eigenvalue, $n=m=3$, we get a closed expression for the critical curve,

$$
\begin{aligned}
& U_{\mathrm{s}}^{*} \approx \\
& \frac{2 R_{3,3} \mathcal{N}_{3} \mathcal{D}_{3}-\mathcal{D}_{1}+\sqrt{\mathcal{D}_{1}^{2}+4 R_{3,3} \mathcal{D}_{3}\left(\mathcal{N}_{1} \mathcal{D}_{3}-\mathcal{D}_{1} \mathcal{N}_{3}\right)}}{2 R_{3,3} \mathcal{D}_{3}^{2}}
\end{aligned}
$$

or by expanding the square root,

$$
U_{\mathrm{s}}^{*} \approx \frac{\mathcal{N}_{1}}{\mathcal{D}_{1}}-\frac{Q_{3,3}^{1}\left(\mathcal{N}_{1} \mathcal{D}_{3}-\mathcal{D}_{1} \mathcal{N}_{3}\right)^{2}}{\mathcal{D}_{1}^{3}\left(\lambda_{1}-2 \lambda_{3}\right)},
$$

the coefficients in which are defined by 46 and (51).

The resulting approximations of the critical curves are shown in fig. 3(c) (together with the a priori bound $\underline{U_{\mathrm{s}}^{*}}=\theta$ given by (63)). Comparing those with panel $\overline{(b)}$, we observe that whereas there is little difference for larger $\theta$ (the linear approximation for those was already reasonably good), there is noticeable improvement for $\theta=0.05$ and $\theta=0.15$, where the quadratic correction term in 70 is more significant due to the relatively small denominator $\left(\lambda_{1}-2 \lambda_{3}\right)$.

\section{MCKEAN EQUATION}

\section{A. Model formulation}

Our second example is a piece-wise linear version of the ZFK equation, considered first by McKean in [40] and then also in 42]:

$$
\begin{aligned}
& d=1, \quad \mathbf{D}=(1), \quad \mathbf{u}=(u), \\
& \mathbf{f}(\mathbf{u})=(f(u)), \quad f(u)=-u+\mathrm{H}(u-a),
\end{aligned}
$$

where we assume that $a \in(0,1 / 2)$, and $\mathrm{H}(\cdot)$ is the Heaviside step function. This model is a variant of ZFK, but with a special feature that makes it similar to the front model we consider later: the discontinuity of the kinetic 
term. One of the practical issues caused by this discontinuity is that direct numerical simulations based on finite differences change qualitatively the behaviour of the system: the discretized critical nucleus solution, defined as an even, spatially nontrivial, stationary solution of the discretized equation, may not be unique and becomes stable, whereas in the differential equation it is unique and unstable. This phenomenon is akin to "propagation block" observed in discretized equations of the ZFK and McKean type and discussed e.g. by Keener [43, with the exception is that here we are dealing with even solutions and spatially localized solutions (when extended to the whole line), as opposed to the trigger front solutions which are traditionally the object of interest in the context of propagation block. Keener's result is about a generic system with smooth right-hand sides, and it predicts "frozen solutions" for sufficiently large discretization steps. As we discuss in Appendix A, for the McKean model with its discontinous right-hand side, the situation is different in that the frozen solutions, at least formally, exist for all discretization steps, which motivates the use of a finite-element approximation, both in the direct numerical simulations for calculating the critical curve, and in the hybrid-method determination of the ignition mode. The finite element approach is discussed in Appendix C.

The critical nucleus solution in this equation is found exactly in a closed form,

$$
\hat{u}(x)= \begin{cases}1-(1-a) \frac{\cosh (x)}{\cosh \left(x_{*}\right)}, & x \leq x_{*}, \\ a \exp \left(x_{*}-x\right), & x \geq x_{*},\end{cases}
$$

where

$$
x_{*}=\frac{1}{2} \ln \left(\frac{1}{1-2 a}\right) .
$$

This solution is illustrated in fig. 6(a).

\section{B. Linear theory}

Due to the discontinuity of the right-hand side, the linearization operator becomes singular, i.e. it contains the Dirac delta function (see Appendix B for a discussion):

$$
\mathcal{L} \triangleq \frac{\partial^{2}}{\partial x^{2}}-1-\frac{1}{a} \delta\left(x-x_{*}\right)
$$

In the even-function extended problem, this is identical to the classical problem of a double-well potential in quantum mechanics. In the space of bounded functions, this operator has one positive eigenvalue. This eigenvalue and the corresponding eigenfunction can be written in the form

$$
\begin{aligned}
& \lambda_{1}=-1+\kappa^{2}, \\
& V_{1}=\left\{\begin{array}{l}
\frac{\cosh (\kappa x)}{\cosh \left(\kappa x_{*}\right)}, \quad x \leq x_{*}, \\
\exp \left(\kappa\left(x_{*}-x\right)\right), \quad x \geq x_{*},
\end{array}\right.
\end{aligned}
$$

where

$$
\kappa=\frac{1}{2 a}+\frac{1}{2 x_{*}} \mathrm{~W}_{0}\left(\frac{x_{*}}{a} \mathrm{e}^{-x_{*} / a}\right)
$$

and $\mathrm{W}_{0}(\cdot)$ is the principal branch of the Lambert $\mathrm{W}$ function as defined e.g. in 44. The behaviour of this eigenpair at different values of $a$ is illustrated in fig. 6(b,c).

Substituting (73), 74), (76), 777) into $(26)$, we obtain the analytical expression for the strength-extent curve,

$$
U_{\mathrm{s}}^{*}\left(x_{\mathrm{s}}\right)=\left\{\begin{array}{l}
\frac{\kappa \mathcal{N}}{\sinh \left(\kappa x_{\mathrm{s}}\right)}, \quad x_{\mathrm{s}}<x_{*}, \\
\frac{\kappa \mathcal{N}}{\sinh \left(\kappa x_{\mathrm{s}}\right)-\cosh \left(\kappa x_{\mathrm{s}}\right)\left(\mathrm{e}^{\kappa\left(x_{*}-x_{\mathrm{s}}\right)}-1\right)}, \\
x_{\mathrm{s}}>x_{*} .
\end{array}\right.
$$

This prediction is compared with the direct numerical simulations in fig. 7 (a). In this case, the theoretical prediction at larger $x_{\mathrm{s}}$ falls below the apriori bound $U_{\mathrm{s}}^{*}=a$, so is "easily improved" by applying this bound. This is also shown in the figure.

In this model, since the exact analytical solution for the critical nucleus and the ignition eigenpair is known for an arbitrary $a \in(0,1 / 2)$, the "hybrid approach" is not necessary. For technical purposes we have tried it as well, and when used with finite-element discretization it works satisfactorily; but since it does not offer any extra insights, we do not present those results here.

\section{Quadratic theory}

The stable spectrum of the linearized problem in this case is entirely continuous, comprising all $\lambda \leq-1$ (see e.g. [20]), with the corresponding generalized eigenfunctions in the form

$V(x ; \lambda)=\rho \cos (\rho x)-\cos \left(\rho x_{*}\right) \sin \left(\rho\left(x-x_{*}\right)\right) \mathrm{H}\left(x-x_{*}\right)$

where $\rho=\sqrt{-1-\lambda}$.

Hence the sums in $m, n$ in 49 are to be interpreted as integrals over $\lambda \in(-\infty,-1]$. The ensuing expressions are rather complicated and whereas it is plausible that the results can be expressed in a closed form, this goes well beyond the scope of this paper, and is left for another study. For now, as a proof-of-principle study, we have obtained a quadratic approximation of the critical curve, by restricting the infinite interval $x \in[0, \infty)$ to a finite interval $x \in[0, L]$, with a homogeneous Dirichlet boundary condition at $x=L$, thus making the spectrum discrete, and truncating the infinite sums in (49) to a finite number of terms. A represenative result is shown in fig. 7(b). This was obtained for $L=10$ and 287 eigenvalues. 


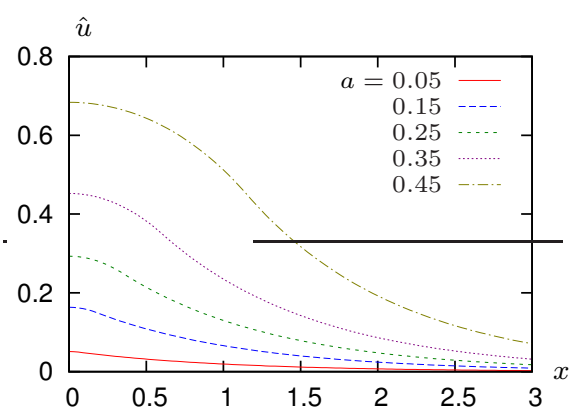

(a)

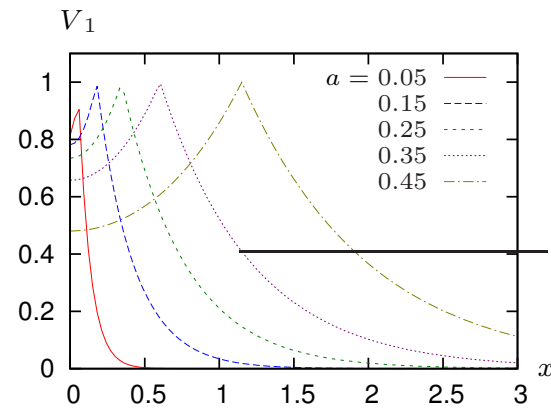

(b)

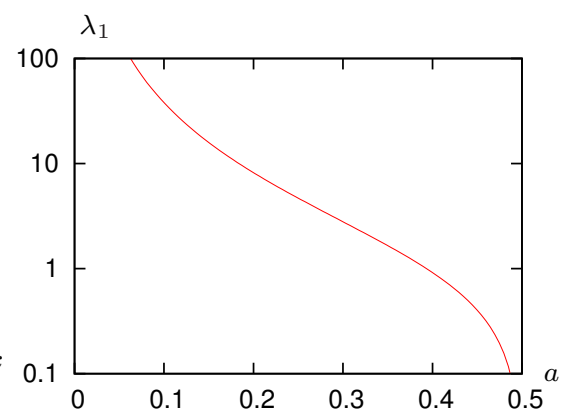

(c)

FIG. 6: (Color online) (a) Critical nucleus solutions, (b) ignition modes and (c) eigenvalues of the McKean model 72 for various values of the parameter $a$.

FIG. 7: (Color online) Strength-extent curves in McKean model: direct numerical simulations (red crosses) vs (a) linear theory, for $a=0.05$ at the bottom increased by 0.1 to $a=0.45$ at the top, and (b) linear and quadratic theories, for $a=0.48$. Blue longdashed lines: analytical dependencies given by 78 . Green short-dashed lines: the lower bound $U_{\mathrm{s}}=a$ in (a) and the predictions given by quadratic theory in (b). Discretization: $\Delta_{x}=0.01, \Delta_{t}=4 \Delta_{x}^{2} / 9, L=10$.

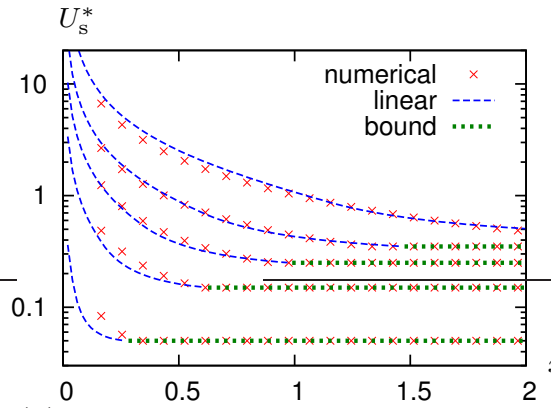

(a)

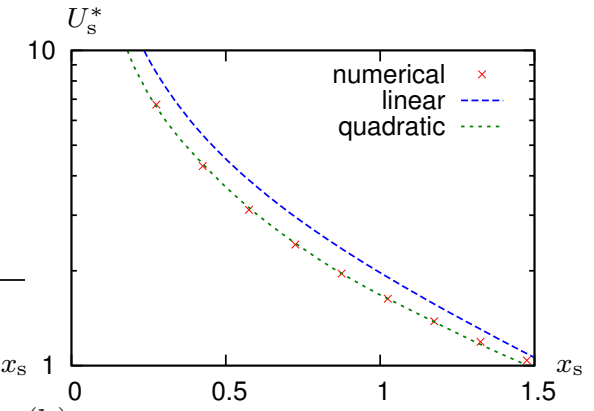

(b)

\section{THE CARICATURE MODEL OF THE $I_{\mathrm{Na}}$-DRIVEN CARDIAC EXCITATION FRONT}

\section{A. Model formulation}

Our next example is the caricature model of an $I_{\mathrm{Na}^{-}}$ driven cardiac excitation front suggested in [45. It is a two-component reaction-diffusion system (1) with $\mathbf{u}=$ $(E, h)^{\top}, \mathbf{D}=\left(\begin{array}{ll}1 & 0 \\ 0 & 0\end{array}\right)$ and $\mathbf{f}=\left(f_{E}, f_{h}\right)^{\top}$, where

$$
\begin{aligned}
f_{E}(E, h) & =\mathrm{H}(E-1) h, \\
f_{h}(E, h) & =\frac{1}{\tau}(\mathrm{H}(-E)-h),
\end{aligned}
$$

and $\mathrm{H}(\cdot)$ is the Heaviside step function. The component $E$ of the solution corresponds to the nondimensionalized transmembrane voltage, and the component $h$ describes the inactivation gate of the fast sodium current, which is known in electrophysiology as $I_{\mathrm{Na}}$ and which is mainly responsible for the propagation of excitation in cardiac muscle in the norm.

A special feature of this model is that there is a continuum of potential resting/pre-front states,

$$
\mathbf{u}_{r}=\lim _{\xi \rightarrow \infty} \hat{\mathbf{u}}=(-\alpha, 1)^{\top}, \quad \alpha>0,
$$

and a continuum of potential post-front states,

$$
\mathbf{u}_{-}=\lim _{\xi \rightarrow-\infty} \hat{\mathbf{u}}=(\omega, 0)^{\top}, \quad \omega>1
$$

so any front solution connects a point from one continuum to a point from the other continuum.

The critical solution $\hat{\mathbf{u}}=(\hat{E}, \hat{h})^{\top}$ is described by

$$
\begin{aligned}
& \hat{E}(\xi)= \begin{cases}\omega-\frac{\tau^{2} c^{2}}{1+\tau c^{2}} \mathrm{e}^{\xi /(\tau c)}, & \xi \leq-\Delta, \\
-\alpha+\alpha \mathrm{e}^{-c \xi}, & \xi \geq-\Delta,\end{cases} \\
& \hat{h}(\xi)= \begin{cases}\mathrm{e}^{\xi /(\tau c)}, & \xi \leq 0, \\
1, & \xi \geq 0,\end{cases}
\end{aligned}
$$

where the post-front voltage $\omega$ and the front thickness $\Delta$ are given by

$$
\omega=1+\tau c^{2}(1+\alpha), \quad \Delta=\frac{1}{c} \ln \left(\frac{1+\alpha}{\alpha}\right),
$$

and the front speed $c$ is defined by an implicit equation

$$
\tau c^{2} \ln \left(\frac{(1+\alpha)\left(1+\tau c^{2}\right)}{\tau}\right)+\ln \left(\frac{\alpha+1}{\alpha}\right)=0,
$$

or equivalently

$$
\tau=g(\beta, \sigma) \triangleq \frac{1+\sigma}{1-\beta} \beta^{-1 / \sigma}
$$

where

$$
\sigma=\tau c^{2}, \beta=\alpha /(\alpha+1)
$$




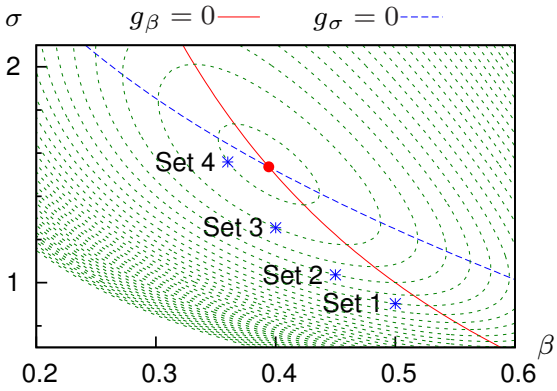

FIG. 8: (Color online) Solutions of $(83)$ for $\tau=7.7$ and above with step 0.1 . The dot is the global minimum of $g(\beta, \sigma)$, the asterisks show the selected sets of parameters in the front model.

Solutions of the transcendental equation $(83)$ are illustrated in fig. 8. As shown in [45, for every $\tau>\tau_{*} \approx 7.674$ there is an interval of values of $\alpha$, in which there are two solutions for $c$. The larger $c$ corresponds to the stable, taller propagating front, and the smaller $c$ corresponds to the unstable, lower propagating front. Our previous numerical simulations [27] indicated that the unstable front is the critical solution in this system: this unstable front is observed as a long-time transient for near-threshold initial conditions from different one-parametric families (corresponding to stimuli of different widths and varying magnitudes), which is a phenomenological evidence that its center-stable manifold has codimension one.

We stress again that in this caricature model, the prefront voltage $-\alpha$ is a parameter of the solution, rather than of the model, and for every $\tau$ large enough, can take any value from an interval. In other words, each such resting state is not an isolated equilibrium, but is a member of a continuous, one-parametric family of equilibria [59].

The a priori bound 63 for the ignition threshold, discussed in Section IIE is not applicable to the present two-component model. However, it is easy to see that if the initial condition $E(x, 0)<1$ for all $x$, then the equation for $E$ reduces to a diffusion equation, and ignition of a propagating wave is therefore out of question. Since the initial perturbation is to be applied to the resting state $E_{\mathrm{r}}=-\alpha$, we conclude that here there is a lower bound for the ignition threshold

$$
\underline{U_{\mathrm{s}}^{*}}=1+\alpha,
$$

which again can be used in conjunction with analytical predictions obtained from other considerations.

\section{B. Linearized problem and eigenfunctions}

The linearized operator (17) requires the Jacobian $\mathbf{F}(\xi)$ defined by (16). Formal differentiation of the Heaviside functions in the kinetic terms using the chain rule (see
Appendix B produces

$$
\begin{gathered}
{\left[\frac{\partial \mathrm{H}(E-1)}{\partial E}\right]_{E=\hat{E}}=\frac{\partial \mathrm{H}(-\Delta-\xi)}{\partial \xi} / \frac{\partial \hat{E}}{\partial \xi}} \\
=-\frac{1}{\hat{E}^{\prime}(-\Delta)} \delta(\xi+\Delta), \\
{\left[\frac{\partial \mathrm{H}(-E)}{\partial E}\right]_{E-\hat{E}}=\frac{\partial \mathrm{H}(\xi)}{\partial \xi} / \frac{\partial \hat{E}}{\partial \xi}=\frac{1}{\hat{E}^{\prime}(0)} \delta(\xi),}
\end{gathered}
$$

then 79 gives for the Jacobian 16):

$$
\mathbf{F}(\xi)=\left(\begin{array}{cc}
-\frac{1}{\hat{E}^{\prime}(-\Delta)} \delta(\xi+\Delta) & \mathrm{H}(-\Delta-\xi) \\
\frac{1}{\tau \hat{E}^{\prime}(0)} \delta(\xi) & -\frac{1}{\tau}
\end{array}\right)
$$

Hence the linearized equations 15 for $\mathbf{v}=\left(E_{1}, h_{1}\right)$ are, componentwise,

$$
\begin{aligned}
\frac{\partial E_{1}}{\partial \tau} & =\frac{\partial^{2} E_{1}}{\partial \xi^{2}}+c \frac{\partial E_{1}}{\partial \xi} \\
& -\frac{1}{\hat{E}^{\prime}(-\Delta)} \delta(\xi+\Delta) \hat{h} E_{1}+\mathrm{H}(-\Delta-\xi) h_{1}, \\
\frac{\partial h_{1}}{\partial \tau} & =c \frac{\partial h_{1}}{\partial \xi}+\frac{1}{\tau \hat{E}^{\prime}(0)} \delta(\xi) E_{1}-\frac{1}{\tau} h_{1} .
\end{aligned}
$$

The spatial operator in 860 is of the third order, so the linear eigenvalue problem (18) can be cast into a thirdorder ODE system for $\phi, \chi$ and $\psi$, where $\mathbf{V}=(\phi, \psi)^{\top}$ and $\chi=\mathrm{d} \phi / \mathrm{d} \xi$, which can be written in the matrix form as

$$
\frac{\mathrm{d} \boldsymbol{\Xi}}{\mathrm{d} \xi}=\mathbf{A} \boldsymbol{\Xi},
$$

where $\boldsymbol{\Xi}=(\phi, \chi, \psi)^{\top}$ and

$$
\mathbf{A}=\left(\begin{array}{ccc}
0 & 1 & 0 \\
\lambda+\frac{\delta(\xi+\Delta)}{\hat{E}^{\prime}(-\Delta)} & -c & -\mathrm{H}(-\xi-\Delta) \\
\frac{-\delta(\xi)}{\tau c \hat{E}^{\prime}(0)} & 0 & \frac{1+\lambda \tau}{\tau c}
\end{array}\right)
$$

The regular part of matrix 88 is piecewise constant, hence the general solution to (87) can be written as

$$
\boldsymbol{\Xi}(\xi)=\sum_{m=1}^{3} i_{m} \mathbf{q}_{m}^{i} \exp \left(\mu_{m}^{i} \xi\right), \quad \xi \in I_{i},
$$

where symbol $i$ takes one of three symbolic values, $i=$ $a, b, c$, designating intervals $I_{a}=(\infty,-\Delta), I_{b}=(-\Delta, 0)$, $I_{c}=(0, \infty)$, the vectors $\mathbf{q}_{m}^{a}, \mathbf{q}_{m}^{b}, \mathbf{q}_{m}^{c}, m=1,2,3$ are the eigenvectors of $\mathbf{A}$ in these intervals, $\mu_{m}^{a}, \mu_{m}^{b}, \mu_{m}^{c}$, are 
the corresponding eigenvalues, and $a_{m}, b_{m}, c_{m}$ are coefficients of the solution in the bases of those eigenvectors in each of the intervals. We have, for $\lambda \geq 0$,

$$
\begin{array}{ll}
\mu_{1}^{i}=\frac{1+\lambda \tau}{\tau c} & =\nu_{1}(\lambda)>0, \\
\mu_{2}^{i}=\frac{-c-\sqrt{c^{2}+4 \lambda}}{2} & =-\nu_{2}(\lambda)<0, \\
\mu_{3}^{i}=\frac{-c+\sqrt{c^{2}+4 \lambda}}{2} & =\nu_{3}(\lambda) \geq 0,
\end{array}
$$

for all three intervals $i=a, b, c$. For the sake of brevity, in the rest of this section we keep the dependence of $\nu_{1,2,3}$ on $\lambda$ in mind, but omit in writing.

Boundary conditions $\boldsymbol{\Xi}( \pm \infty)=0[60$ then require that $a_{2}=c_{1}=c_{3}=0$, and by finding eigenvectors of $\mathbf{A}$ in the three intervals, we have

$$
\left(\begin{array}{c}
\phi \\
\chi \\
\psi
\end{array}\right)=\left\{\begin{array}{rr}
a_{1}\left(\begin{array}{c}
1 \\
\nu_{1} \\
-q
\end{array}\right) \mathrm{e}^{\nu_{1} \xi}+a_{3}\left(\begin{array}{c}
1 \\
\nu_{3} \\
0
\end{array}\right) \mathrm{e}^{\nu_{3} \xi}, & \xi \in I_{a}, \\
b_{1}\left(\begin{array}{c}
0 \\
0 \\
1
\end{array}\right) \mathrm{e}^{\nu_{1} \xi}+b_{2}\left(\begin{array}{c}
1 \\
-\nu_{2} \\
0
\end{array}\right) \mathrm{e}^{-\nu_{2} \xi}+b_{3}\left(\begin{array}{c}
1 \\
\nu_{3} \\
0
\end{array}\right) \mathrm{e}^{\nu_{3} \xi}, \\
\xi \in I_{b}, \\
c_{2}\left(\begin{array}{c}
1 \\
-\nu_{2} \\
0
\end{array}\right) \mathrm{e}^{-\nu_{2} \xi}, \\
\xi \in I_{c},
\end{array}\right.
$$

where

$$
q=\nu_{1}\left(\nu_{1}+c\right)-\lambda=\left(\frac{1+\lambda \tau}{\tau c}\right)^{2}+\frac{1}{\tau}>0
$$

These solutions are to be matched at $\xi=-\Delta$ and $\xi=0$, with account of the singular terms in matrix (88). Using notation $[\cdot]$ for a jump of a function at a point, the matching conditions for 87, 88, can be written as

$$
\begin{aligned}
& {[\chi(-\Delta)]=\frac{\phi(-\Delta)}{\hat{E}^{\prime}(-\Delta)},} \\
& {[\psi(0)]=-\frac{\phi(0)}{\tau c \hat{E}^{\prime}(0)},}
\end{aligned}
$$

and we have continuity in all other cases,

$$
[\phi(-\Delta)]=[\psi(-\Delta)]=[\phi(0)]=[\chi(0)]=0
$$

For the solution 89 , this amounts to the following alge- braic system for the coefficients:

$$
\begin{aligned}
& a_{1} \mathrm{e}^{-\nu_{1} \Delta}+a_{3} \mathrm{e}^{-\nu_{3} \Delta}-b_{2} \mathrm{e}^{\nu_{2} \Delta}-b_{3} \mathrm{e}^{-\nu_{3} \Delta}=0 \\
& a_{1} \alpha c \nu_{1} \mathrm{e}^{-\nu_{1} \Delta}+a_{3} \alpha c \nu_{3} \mathrm{e}^{-\nu_{3} \Delta}+b_{2} \mathrm{e}^{\nu_{2} \Delta}\left(\alpha c \nu_{2}-\mathrm{e}^{-\nu \Delta}\right) \\
& \quad-b_{3} \mathrm{e}^{-\nu_{3} \Delta}\left(\alpha c \nu_{3}+\mathrm{e}^{-\nu \Delta}\right)=0 \\
& a_{1} q+b_{1}=0 \\
& b_{2}+b_{3}-c_{2}=0 \\
& b_{2} \nu_{2}-b_{3} \nu_{3}-c_{2} \nu_{2}=0 \\
& b_{1} \alpha \tau c^{2}+c_{2}=0
\end{aligned}
$$

where

$$
\nu=\frac{1+\tau c^{2}}{\tau c}>0 .
$$

The solvability condition for this system is given by the roots of function $f_{e}(\lambda ; \ldots)$ (proportional to the Evans function) defined as

$$
\begin{aligned}
f_{e}(\lambda ; c, \alpha, \tau) & \triangleq \alpha c\left(\nu_{2}+\nu_{3}\right) \mathrm{e}^{\nu \Delta}-1 \\
& +\frac{\tau c\left(\nu_{1}-\nu_{3}\right)}{(1+\lambda \tau)^{2}+\tau c^{2}} \mathrm{e}^{\left(\nu-\nu_{1}-\nu_{2}\right) \Delta}=0,
\end{aligned}
$$

and the solution, up to normalization, is

$$
\begin{aligned}
& a_{1}=1, \\
& a_{3}=\alpha \tau c^{2} q \mathrm{e}^{\left(\nu_{2}+\nu_{3}\right) \Delta}-\mathrm{e}^{\left(\nu_{3}-\nu_{1}\right) \Delta}, \\
& b_{1}=-q, \\
& b_{2}=\alpha \tau c^{2} q, \\
& b_{3}=0, \\
& c_{2}=\alpha \tau c^{2} q .
\end{aligned}
$$

The adjoint problem to 86 is

$$
\mathcal{L}^{+} \mathbf{W}=\lambda \mathbf{W}
$$

where

$$
\mathcal{L}^{+}=\mathbf{D}^{\top} \frac{\mathrm{d}^{2}}{\mathrm{~d} \xi^{2}}-c \frac{\mathrm{d}}{\mathrm{d} \xi}+\mathbf{F}^{\top}(\xi), \quad \mathbf{W}=(\tilde{\phi}, \tilde{\psi})^{\top}
$$

and

$$
\mathbf{F}^{\top}(\xi)=\left(\begin{array}{cc}
-\frac{1}{\hat{E}^{\prime}(-\Delta)} \delta(\xi+\Delta) & \frac{1}{\tau \hat{E}^{\prime}(0)} \delta(\xi) \\
\mathrm{H}(-\xi-\Delta) & -\frac{1}{\tau}
\end{array}\right) .
$$

Proceeding as in the previous case, we have a third-order system

$$
\frac{\mathrm{d} \tilde{\boldsymbol{\Xi}}}{\mathrm{d} \xi}=\tilde{A} \tilde{\Xi}
$$

for $\tilde{\boldsymbol{\Xi}}=(\tilde{\phi}, \tilde{\chi}, \tilde{\psi})^{\top}, \tilde{\chi} \equiv \tilde{\phi}^{\prime}$, with the matrix

$$
\tilde{A}=\left(\begin{array}{ccc}
0 & 1 & 0 \\
\lambda+\frac{\delta(\xi+\Delta)}{\hat{E}^{\prime}(-\Delta)} & c & \frac{-\delta(\xi)}{\tau c \hat{E}^{\prime}(0)} \\
\frac{1}{c} \mathrm{H}(-\xi-\Delta) & 0 & -\frac{(1+\lambda \tau)}{\tau c}
\end{array}\right),
$$


its piece-wise solution

$$
\left(\begin{array}{c}
\tilde{\phi}(\xi) \\
\tilde{\chi}(\xi) \\
\tilde{\psi}(\xi)
\end{array}\right)=\left\{\begin{array}{r}
\tilde{a}_{2}\left(\begin{array}{c}
1 \\
\nu_{2} \\
p
\end{array}\right) \mathrm{e}^{\nu_{2} \xi}, \\
\tilde{b}_{1}\left(\begin{array}{c}
0 \\
0 \\
1
\end{array}\right) \mathrm{e}^{-\nu_{1} \xi}+\tilde{b}_{2}\left(\begin{array}{c}
1 \\
\nu_{2} \\
0
\end{array}\right) \mathrm{e}^{\nu_{2} \xi} \\
+\tilde{b}_{3}\left(\begin{array}{c}
1 \\
-\nu_{3} \\
0 \\
1
\end{array}\right) \mathrm{e}^{-\nu_{3} \xi}, \xi \in I_{b}, \\
\tilde{c}_{1}\left(\begin{array}{l}
0 \\
0 \\
1
\end{array}\right) \mathrm{e}^{-\nu_{1} \xi}+\tilde{c}_{3}\left(\begin{array}{c}
\nu_{3} \\
0
\end{array}\right) \mathrm{e}^{-\nu_{3} \xi}, \xi \in I_{c},
\end{array}\right.
$$

where

$$
p=\frac{1}{c\left(\nu_{1}+\nu_{2}\right)},
$$

the algebraic system for the coefficients stemming from the matching conditions,

$$
\begin{aligned}
& \tilde{a}_{2} \mathrm{e}^{-\nu_{2} \Delta}-\tilde{b}_{2} \mathrm{e}^{-\nu_{2} \Delta}-\tilde{b}_{3} \mathrm{e}^{\nu_{3} \Delta}=0, \\
& \tilde{a}_{2} \alpha c \nu_{2} \mathrm{e}^{-\nu_{2} \Delta}-\tilde{b}_{2} \mathrm{e}^{-\nu_{2} \Delta}\left(\alpha c \nu_{2}+\mathrm{e}^{-\nu \Delta}\right) \\
& -\tilde{b}_{3} \mathrm{e}^{\nu_{3} \Delta}\left(-\alpha c \nu_{3}+\mathrm{e}^{-\nu \Delta}\right)=0, \\
& \tilde{a}_{2} p \mathrm{e}^{-\nu_{2} \Delta}-\tilde{b}_{1} \mathrm{e}^{\nu_{1} \Delta}=0 \text {, } \\
& \tilde{b}_{2}+\tilde{b}_{3}-\tilde{c}_{3}=0 \text {, } \\
& \tilde{b}_{2} \alpha \tau c \nu_{2}-\tilde{b}_{3} \alpha \tau c \nu_{3}+\tilde{c}_{1}+\tilde{c}_{3} \alpha \tau c \nu_{3}=0 \text {, } \\
& \tilde{b}_{1}-\tilde{c}_{1}=0 \text {, }
\end{aligned}
$$

the solution of which, up to normalization, is

$\tilde{a}_{2}=\alpha \tau c^{2}\left(\nu_{1}+\nu_{2}\right)\left(\nu_{2}+\nu_{3}\right) \mathrm{e}^{\left(\nu_{1}+\nu_{2}\right) \Delta}$,

$\tilde{b}_{1}=\alpha \tau c\left(\nu_{2}+\nu_{3}\right)$,

$\tilde{b}_{2}=-1$,

$\tilde{b}_{3}=\alpha \tau c^{2}\left(\nu_{1}+\nu_{2}\right)\left(\nu_{2}+\nu_{3}\right) \mathrm{e}^{\left(\nu_{1}-\nu_{3}\right) \Delta}+\mathrm{e}^{-\left(\nu_{2}+\nu_{3}\right) \Delta}$,

$\tilde{c}_{1}=\alpha \tau c\left(\nu_{2}+\nu_{3}\right)$,

$\tilde{c}_{3}=\alpha \tau c^{2}\left(\nu_{1}+\nu_{2}\right)\left(\nu_{2}+\nu_{3}\right) \mathrm{e}^{\left(\nu_{1}-\nu_{3}\right) \Delta}+\mathrm{e}^{-\left(\nu_{2}+\nu_{3}\right) \Delta}-1$,

and its solvability condition is

$$
\tilde{f}_{e}=\alpha c\left(\nu_{2}+\nu_{3}\right)+\frac{\mathrm{e}^{-\left(\nu_{1}+\nu_{2}\right) \Delta}}{\tau c\left(\nu_{1}+\nu_{2}\right)}-\mathrm{e}^{-\nu \Delta}=0 .
$$

The solvability condition $\sqrt{103}$ is equivalent to $(93)$, the characteristic equation of the linearized problem. Its dependence on $\lambda$ is implicit via $\nu_{1}, \nu_{2}, \nu_{3}, p$. The corresponding explicit expression is a available but too lengthy and we do not present it here. Alternatively, using substitutions 84 and

$$
z=\sqrt{1+4 \lambda / c^{2}}
$$

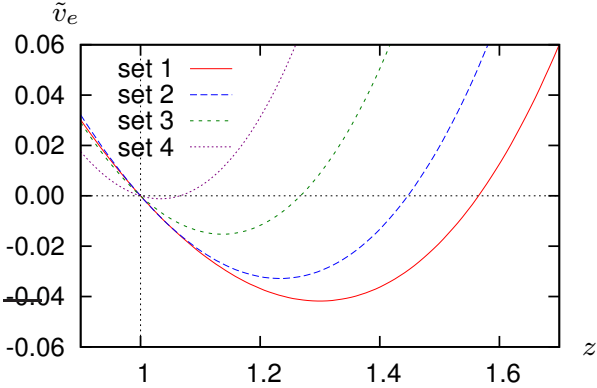

FIG. 9: (Color online) Plots of the charateristic function 105) for the four selected parameter sets.

\begin{tabular}{|l|l|l|l|l|}
\hline & Set 1 & Set 2 & Set 3 & Set 4 \\
\hline$\beta$ & 0.5 & 0.45 & 0.4 & 0.36 \\
\hline$\tau$ & 8.2 & 8 & 7.8 & 7.7 \\
\hline$\sigma$ & 0.903152459 & 1.036565915 & 1.254739882 & 1.559272934 \\
\hline$\alpha$ & 1 & 0.818181818 & 0.666666667 & 0.5625 \\
\hline$c$ & 0.331874289 & 0.359959358 & 0.401078655 & 0.450003309 \\
\hline$\lambda_{1}$ & 0.03990255 & 0.035413196 & 0.024380836 & 0.006905681 \\
\hline
\end{tabular}

TABLE I: Selected sets of parameters and corresponding eigenvalues for the front model.

the characteristic equation is explicitly rewritten in a more compact form,

$$
\begin{aligned}
& 0=\tilde{v}_{e}(z)=\left(\sigma+\left(1+\frac{\sigma\left(z^{2}-1\right)}{4}\right)^{2}\right)\left(\frac{\sigma z}{1+\sigma}-1\right) \\
& +\left(1+\frac{\sigma(z-1)^{2}}{4}\right) \beta^{(1+z)^{2} / 4-1} .
\end{aligned}
$$

Fig. 9 illustrates the behaviour of the function $\tilde{v}_{e}(z)$ defined (105) for selected values of parameters $\beta$ and $\sigma$, indicated by asterisks in fig. 8. The roots $z>1$ of (105) define the positive eigenvalues $\lambda_{1}$, and, of course, in all cases $\lambda_{2}=0$ which corresponds to $z=1$. Numerical values of $\lambda_{1}$ for the four selected sets of parameters are presented in Table I]

Knowing $\lambda_{\ell}, \ell=1,2$, we obtain the adjoint eigenfunctions $\mathbf{W}_{\ell}(\xi), \ell=1,2$, by formulas $(99)$.

\section{Strength-extent curve}

Given the expressions for the adjoint eigenfunctions (99), 102 for our model, we are now in a position to calculate the pre-compatibility function (41) required to obtain the analytical description of the critical curve. For the components of the left eigenfunctions, $\mathbf{W}_{\ell}=$ 

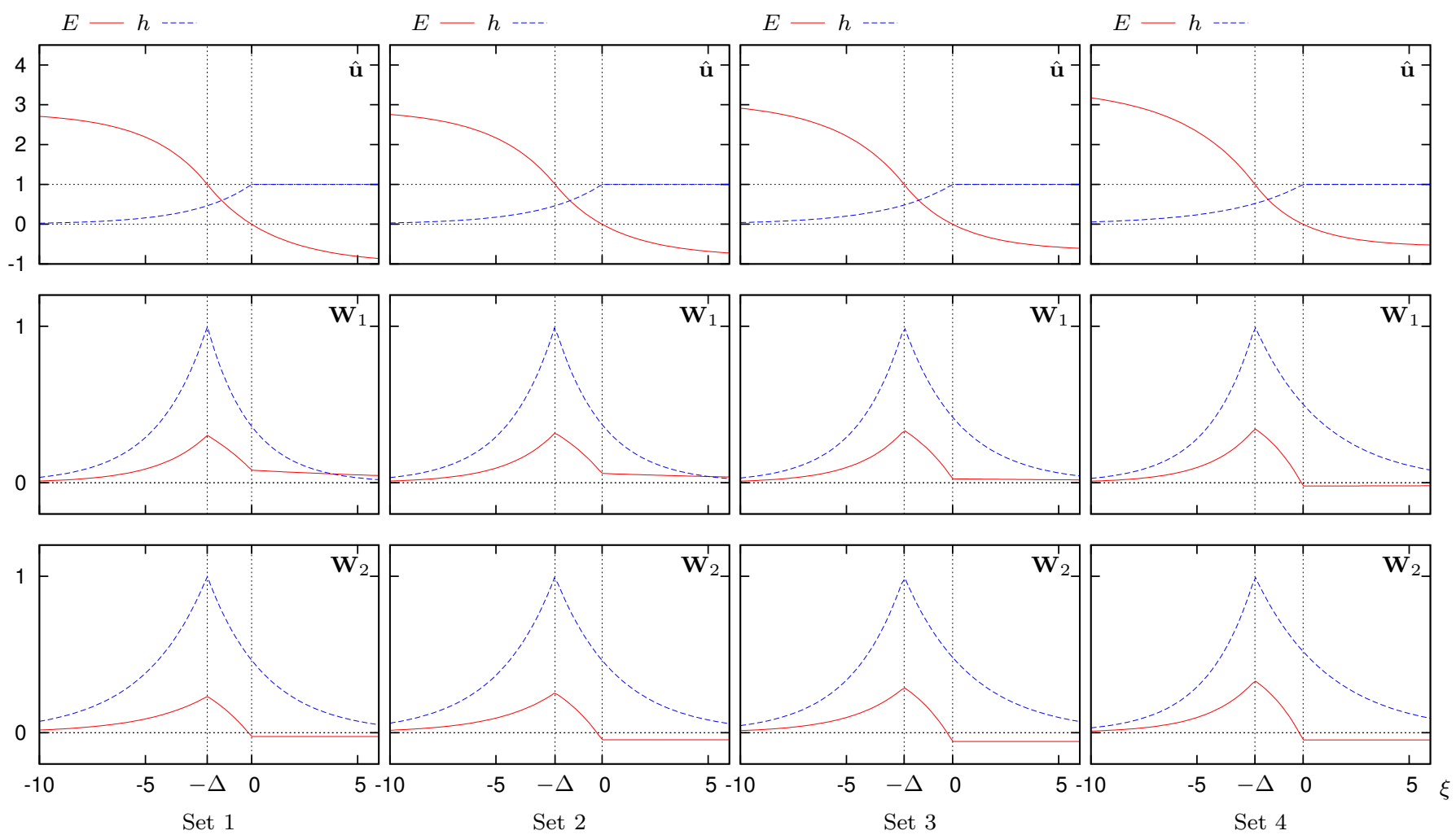

FIG. 10: (Color online) Linearized theory ingredients for the four selected sets of parametes in the front model. Shown are components of vector functions, indicated in top right corner of each panel. The functions $\mathbf{W}_{j}$ are scaled so that maximal value of $h$-component is 1 . Correspondence of lines with components is according to the legends at the top.

$\left(\tilde{\phi}^{\ell}, \tilde{\psi}^{\ell}\right)^{\top}, \ell=1,2$, we have

$$
\begin{gathered}
\tilde{\phi}^{\ell}(\xi)= \begin{cases}\tilde{a}_{2}^{\ell} \mathrm{e}^{\nu_{2}^{\ell} \xi}, & \xi \in I_{a}, \\
\tilde{b}_{2}^{\ell} \mathrm{e}^{\nu_{2}^{\ell} \xi}+\tilde{b}_{3}^{\ell} \mathrm{e}^{-\nu_{3}^{\ell} \xi}, & \xi \in I_{b}, \\
\tilde{c}_{3}^{\ell} \mathrm{e}^{-\nu_{3}^{\ell} \xi}, & \xi \in I_{c},\end{cases} \\
\tilde{\psi}^{\ell}(\xi)= \begin{cases}\tilde{a}_{2}^{\ell} p^{\ell} \mathrm{e}^{\nu_{2}^{\ell} \xi}, & \xi \in I_{a}, \\
\tilde{b}_{1}^{\ell} \mathrm{e}^{-\nu_{1}^{\ell} \xi}, & \xi \in I_{b}, \\
\tilde{c}_{1}^{\ell} \mathrm{e}^{-\nu_{1}^{\ell} \xi}, & \xi \in I_{c} .\end{cases}
\end{gathered}
$$

The resulting $\mathbf{W}_{\ell}(\xi), \ell=1,2$, for the selected values of parameters are shown in fig. 10, against the corresponding critical nucleus solutions 61]. Then 235 gives

$$
\begin{aligned}
& \mathcal{N}_{\ell}=\left\langle\mathbf{W}_{\ell}(\xi) \mid \hat{\mathbf{u}}(\xi)-\mathbf{u}_{r}\right\rangle \\
& =\int_{-\infty}^{\infty}\left(\tilde{\phi}^{\ell}(\xi)\left(\hat{E}(\xi)-E_{\mathrm{r}}\right)+\tilde{\psi}^{\ell}(\xi)\left(\hat{h}(\xi)-h_{\mathrm{r}}\right)\right) \mathrm{d} \xi \\
& =\tilde{a}_{2}^{\ell}\left[\frac{\omega+\alpha-p^{\ell}}{\nu_{2}^{\ell}}+\frac{c(1+\alpha)\left(p^{\ell}\left(1+\tau c^{2}\right)-\tau^{2} c^{2}\right)}{\nu_{2}^{\ell} \tau c+1}\right] \mathrm{e}^{-\nu_{2}^{\ell} \Delta} \\
& +\tilde{b}_{2}^{\ell} \frac{\alpha-(1+\alpha) \mathrm{e}^{-\nu_{2}^{\ell} \Delta}}{\nu_{2}^{\ell}-c}-\tilde{b}_{3}^{\ell} \frac{\alpha-(1+\alpha) \mathrm{e}^{\nu_{3}^{\ell} \Delta}}{\nu_{3}^{\ell}+c} \\
& +\tilde{b}_{1}^{\ell} \tau c \frac{1-\mathrm{e}^{\nu_{1}^{\ell} \Delta} \mathrm{e}^{-\Delta /(\tau c)}}{1-\nu_{1}^{\ell} \tau c}+\tilde{b}_{1}^{\ell} \frac{1-\mathrm{e}^{\nu_{1}^{\ell} \Delta}}{\nu_{1}^{\ell}}+\tilde{c}_{3}^{\ell} \frac{\alpha}{\nu_{3}^{\ell}+c},
\end{aligned}
$$

for $\ell=1,2$. Further, 26 gives

$$
\mathcal{D}(s)=\left\langle\mathbf{W}_{1}(\xi) \mid \mathbf{u}_{\mathrm{s}}(\xi+s)\right\rangle=\int_{-x_{\mathrm{s}}-s}^{x_{\mathrm{s}}-s} \tilde{\phi}^{1}(\xi) \mathrm{d} \xi,
$$

and 42 gives

$$
\mathcal{I}_{\ell}(\xi)=\int \mathbf{e}^{\top} \mathbf{W}_{\ell}(\xi) \mathrm{d} \xi=\int \tilde{\phi}^{\ell}(\xi) \mathrm{d} \xi
$$

$$
=\left\{\begin{array}{cc}
\frac{\tilde{a}_{2}^{\ell}}{\nu_{2}^{\ell}} \mathrm{e}^{\nu_{2}^{\ell} \xi}, & \xi \in I_{a}, \\
\frac{\tilde{a}_{2}^{\ell}-\tilde{b}_{2}^{\ell}}{\nu_{2}^{\ell}} \mathrm{e}^{-\nu_{2}^{\ell} \Delta}+\frac{\tilde{b}_{3}^{\ell}}{\nu_{3}^{\ell}} \mathrm{e}^{\nu_{3}^{\ell} \Delta}+\frac{\tilde{b}_{2}^{\ell}}{\nu_{2}^{\ell}} \mathrm{e}^{\nu_{2}^{\ell} \xi} \\
-\frac{\tilde{b}_{3}^{\ell}}{\nu_{3}^{\ell}} \mathrm{e}^{-\nu_{3}^{\ell} \xi}, & \xi \in I_{b}, \\
\frac{\tilde{a}_{2}^{\ell}-\tilde{b}_{2}^{\ell}}{\nu_{2}^{\ell}} \mathrm{e}^{-\nu_{2}^{\ell} \Delta}+\frac{\tilde{b}_{3}^{\ell}}{\nu_{3}^{\ell}} \mathrm{e}^{\nu_{3}^{\ell} \Delta}+\frac{\tilde{b}_{2}^{\ell}}{\nu_{2}^{\ell}} \\
+\frac{\tilde{c}_{3}^{\ell}-\tilde{b}_{3}^{\ell}}{\nu_{3}^{\ell}}-\frac{\tilde{c}_{3}^{\ell}}{\nu_{3}^{\ell}} \mathrm{e}^{-\nu_{3}^{\ell} \xi}, \quad \xi \in I_{c} .
\end{array}\right.
$$

This general expression works for $\ell=1$; however for $\ell=2$ it fails as $\lambda=0$ and consequently $\nu_{3}^{2}=0$. The expression for $\ell=2$ can be obtained as the $\lambda \rightarrow 0$ limit of the above, 
or by redoing the integration for this special case. Either way, we get

$$
\mathcal{I}_{2}(\xi)=\left\{\begin{array}{cc}
\frac{\tilde{a}_{2}^{2}}{\nu_{2}^{2}} \mathrm{e}^{\nu_{2}^{2} \xi}, & \xi \in I_{a}, \\
\frac{\tilde{a}_{2}^{2}}{\nu_{2}^{2}} \mathrm{e}^{-\nu_{2}^{2} \Delta}-\frac{\tilde{b}_{2}^{2}}{\nu_{2}^{2}} \mathrm{e}^{-\nu_{2}^{2} \Delta}+\frac{\tilde{b}_{2}^{2}}{\nu_{2}^{2}} \mathrm{e}^{\nu_{2}^{2} \xi} & \xi \in I_{b}, \\
+\tilde{b}_{3}^{2}(\Delta+\xi), & \xi \tilde{a}_{2}^{2} \mathrm{e}^{-\nu_{2}^{2} \Delta}-\frac{\tilde{b}_{2}^{2}}{\nu_{2}^{2}} \mathrm{e}^{-\nu_{2}^{2} \Delta}+\frac{\tilde{b}_{2}^{2}}{\nu_{2}^{2}} \\
+\tilde{b}_{3}^{2} \Delta+\tilde{c}_{3}^{2} \xi, & \xi \in I_{c} .
\end{array}\right.
$$

Also, for $\lambda=\lambda_{2}=0, \mathcal{N}_{2}$ simplifies to

$$
\begin{gathered}
\mathcal{N}_{2}=\left\langle\mathbf{W}_{2}(\xi) \mid \hat{\mathbf{u}}(\xi)-\mathbf{u}_{r}\right\rangle \\
=\tilde{a}_{2}^{2} \frac{2 \alpha \tau c^{2}}{c\left(1+\tau c^{2}\right)}+\left(\tilde{a}_{2}^{2} \frac{\alpha}{c}+\tilde{b}_{1}^{2} \tau c\right)\left(1-\frac{\tau}{(1+\alpha)\left(1+\tau c^{2}\right)}\right) \\
+\left(\tilde{b}_{2}^{2} \alpha+\tilde{b}_{1}^{2}\right) \Delta+\tilde{b}_{3}^{2} \frac{1}{c}+\tilde{c}_{3}^{2} \frac{\alpha}{c} .
\end{gathered}
$$

The critical curve is then described based on the function $\eta(\xi)$, defined by 41,

$$
\eta(\xi)=\mathcal{N}_{1} \mathcal{I}_{2}(\xi)-\mathcal{N}_{2} \mathcal{I}_{1}(\xi),
$$

using the implicit-function definition 40-44. Fig. 11 shows the function $\eta(\xi)$ for the four selected parameter sets. It is clearly unimodal in all four cases, however the position of the maximum varies a lot. We note that for set 1 , the position of the maximum is far ahead of the critical front, which has a (rather unlikely) implication that this is where the main events, deciding whether the front will be ignited, take place.

As can be seen from the above, even though the function $\eta(\xi)$ for this example is found explicitly, its form appears too complicated to establish analytically whether it is always unimodal, let alone to explicitly invert it. So we have done the inversion numerically. A comparison of the resulting critical curve against the direct numerical simulations is shown in fig. 12. We see that the accuracy of the theoretical predictions varies considerably, and for Set 4 is very close. We do not have any conclusive explanation of the difference in the accuracy, only note that better approximation is associated with a more reasonable position of the extremum of the pre-compatibility function $\eta(\xi)$ and smaller values of $\lambda_{1}$.

\section{FITZHUGH-NAGUMO SYSTEM}

\section{A. Model formulation}

The FitzHugh-Nagumo (FHN) system is a twocomponent reaction-diffusion system, which could be

\begin{tabular}{|c|c|c|c|}
\hline$\beta$ & $c$ & $\lambda_{1}$ & $\lambda_{2}$ \\
\hline 0.05 & 0.2561 & 0.17204 & $\pm 1 \cdot 10^{-5}$ \\
\hline 0.13 & 0.2328 & 0.18619 & $\pm 1 \cdot 10^{-5}$ \\
\hline
\end{tabular}

TABLE II: Nonlinear and linear eigenvalues for the FitzHughNagumo system.

considered as a ZFK equation extended by adding a second, slow variable, describing inhibition of excitation. It is probably the single historically most important model describing excitable media. We consider it in the form

$$
\begin{aligned}
& d=2, \quad \mathbf{D}=\operatorname{diag}(1,0), \quad \mathbf{u}=(u, v)^{\top}, \\
& \mathbf{f}(\mathbf{u})=\left(f_{u}(u, v), f_{v}(u, v)\right)^{\top}, \\
& f_{u}(u, v)=u(u-\beta)(1-u)-v, \\
& f_{v}(u, v)=\gamma(\alpha u-v) .
\end{aligned}
$$

for fixed values of the slow dynamics parameters, $\gamma=$ 0.01 and $\alpha=0.37$, and two values of the excitation threshold for the fast dynamics, $\beta=0.05$ and $\beta=0.13$.

Unlike the ZFK equation, the critical solution in system 107) is moving, as in the $I_{\mathrm{Na}}$ front model, but it is a critical pulse rather than critical front. It is known (see e.g. 26] and references therein) that in the limit $\gamma \searrow 0$, this system has the critical pulse solution whose $v$-component is small and $u$-component is close to the critical pulse of the corresponding ZFK equation. However this does not provide good enough approximation for the linearized theory, and we used only the hybrid approach. We have obtained the critical pulse by numerical continuation of the periodic pulse problem using AUTO as discussed in Section IID 3. the corresponding $\mathrm{CV}$ restitution curves are illustrated in fig. 13 . For the critical pulses, we take the solutions at lower branches at $P>7.5 \cdot 10^{3}$. The corresponding propagation speeds are given in Table II.

\section{B. Linear theory}

Fig. 14 and Table II illustrate other ingredients required for the semi-analytical prediction of the critical curves for the two selected cases. These are found by the straightforward marching method and then verified by Arnoldi iterations. We use discretization $\Delta_{x}=0.03$, $\Delta_{t}=4 \Delta_{x}^{2} / 9, \xi \in[-L, L], L=100$. As expected, $\left|\lambda_{2}\right|$ are small.

Fig. 15 shows the results of the calculation according to the formulas 40--44). The "pre-compatibility" function $\eta(\xi)$ defined by (41) in both cases is not unimodal, see fig. 15(a), and, at least for $\beta=0.05$, has two local maxima and one local minimum, hence implementation of the algorithm (40)-44) is not straightforward and requires investigation of the local extrema. We find that in both cases the adequate answer is given by the local 
Set 1

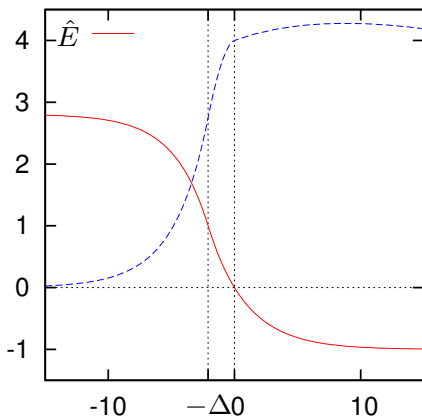

Set 2

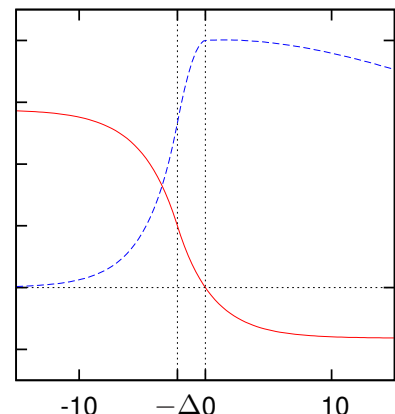

Set 3

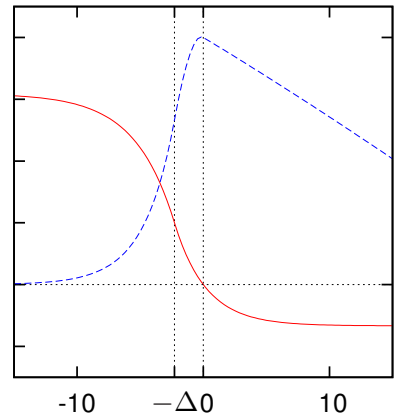

Set 4

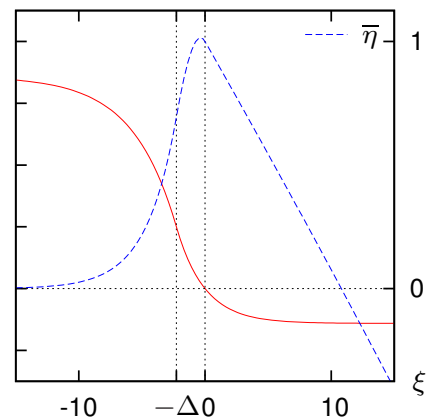

FIG. 11: (Color online) Pre-compatibility functions $\eta(\xi)$ for the four selected sets of parameters in the front model. For visualization purposes, we show the normalized function, $\bar{\eta}(\xi)=\eta(\xi) / \eta(0)$ (dashed lines, right ordinate axes). For positioning, we also show the profile of the corresponding critical front (solid lines, left ordinate axes).
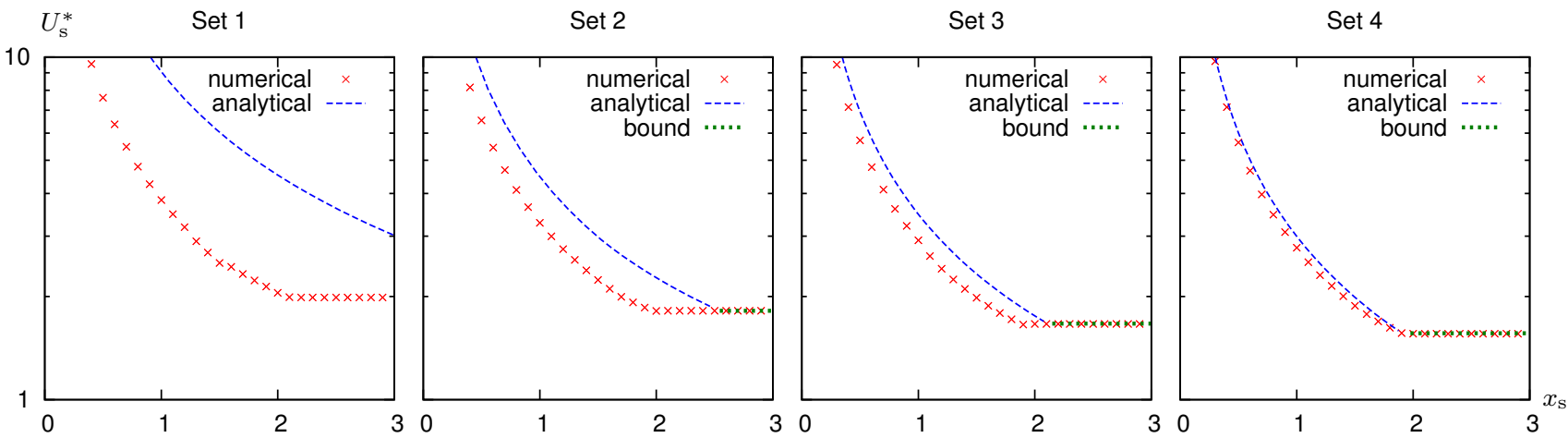

FIG. 12: (Color online) Strength-extent curves in the caricature $I_{\mathrm{Na}}$ front model, for the four selected parameter sets. Shown also is the a priori lower bound for the ignition threshold given by equation (85).

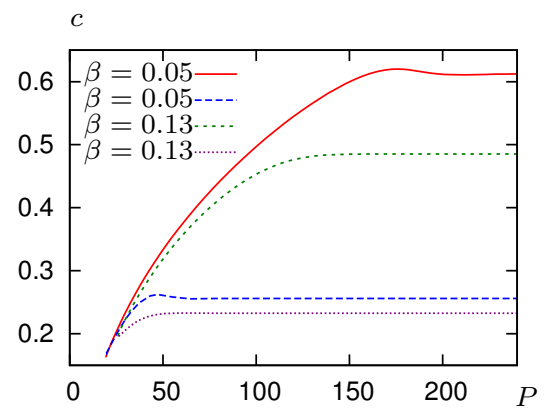

FIG. 13: (Color online) CV restitution curves for the FHN model for two selected values of the model parameter. Stable (upper) and unstable (lower) branches are shown by different line types.

maximum nearest to $\xi=0$, at the front of the critical pulse. The corresponding theoretical critical curves are shown in fig. 15(b), in comparison with the curves obtained by direct numerical simulation. We observe that the theory works somewhat better for $\beta=0.05$ than for $\beta=0.13$, although the eigenfunctions shown in fig. 14 for the two cases look rather similar. Again, the better accuracy of the linearized theory here is associated with smaller value of $\lambda_{1}$, although the relative difference between the two cases is small in itself.

\section{MODIFIED BEELER-REUTER MODEL OF CARDIAC EXCITATION}

\section{A. Model formulation}

Here we look at a variant of the classical BeelerReuter (BR) model of mammalian ventricular cardiac myocytes 46, modified to describe phenomenologically the dynamics of neonatal rat cells 47 -50]:

$$
\begin{gathered}
d=7, \quad \mathbf{D}=\operatorname{diag}(1,0,0,0,0,0,0), \\
\mathbf{u}=\left(V, h, j, x_{1}, d, f, \mathrm{Ca}\right)^{\top}, \\
\mathbf{f}(\mathbf{u})=\left(\begin{array}{c}
-\left(I_{K_{1}}+I_{x 1}+I_{N a}+I_{s}\right) \\
\alpha_{h}(1-h)-\beta_{h} h \\
\alpha_{j}(1-j)-\beta_{j} j \\
\alpha_{x 1}\left(1-x_{1}\right)-\beta_{x 1} x_{1} \\
\alpha_{d}(1-d)-\beta_{d} d \\
\alpha_{f}(1-f)-\beta_{f} f \\
-10^{-7} I_{s}+0.07\left(10^{-7}-\mathrm{Ca}\right)
\end{array}\right) .
\end{gathered}
$$



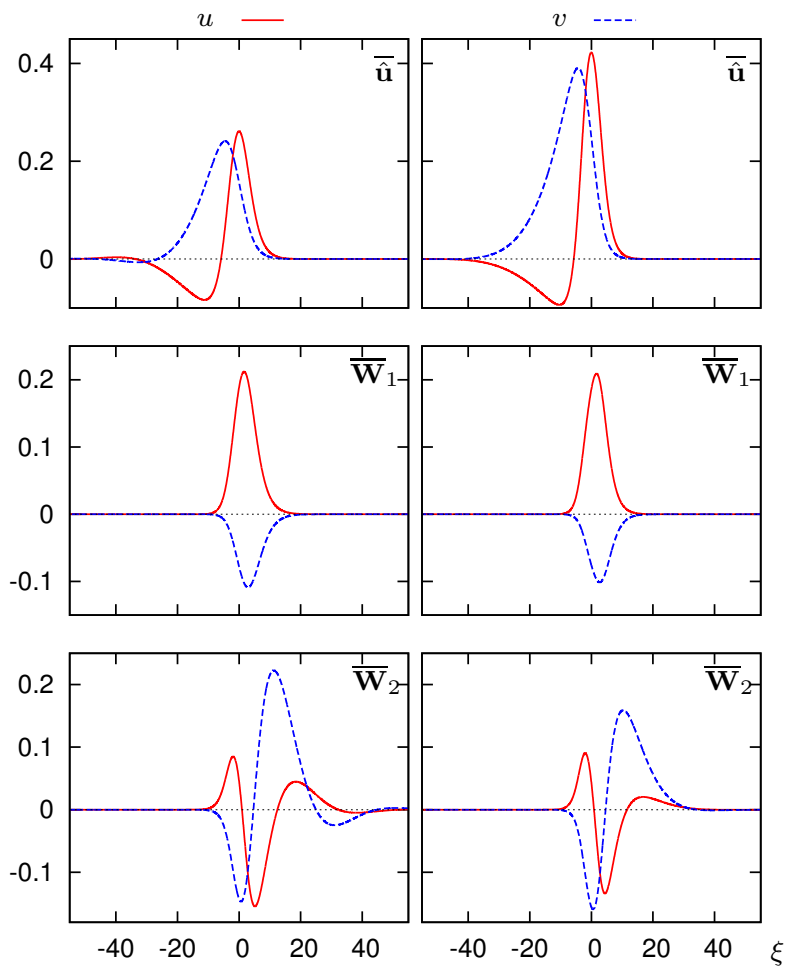

(a)

(b)

FIG. 14: (Color online) FHN theory ingredients for (a) $\beta=$ 0.05 and (b) $\beta=0.13$. Shown are components of scaled vector functions, indicated in top right corner of each panel, where $\overline{\hat{\mathbf{u}}}=\mathbf{S} \hat{\mathbf{u}}, \overline{\mathbf{W}}_{j}=\mathbf{S}^{-1} \mathbf{W}_{j}$, and $\mathbf{S}=\operatorname{diag}(1,10)$. The space coordinate is chosen so that $\xi=0$ at the maximum of $\hat{u}$. Correspondence of lines with components is according to the legends at the top.

The detailed description of the components of $\mathbf{f}(\mathbf{u})$ is given in Appendix D. Important here is only the dependence on the "excitability" parameter $\alpha$, which appears in the equations in the following way:

$$
I_{K_{1}}=0.35(0.3-\alpha) \overline{I_{K_{1}}}(V) .
$$

In 50, special attention was given to $\alpha=0.105$ ("less excitable", with negative filament tension of the scroll waves) and $\alpha=0.115$ ("more excitable", with positive filament tension of the scroll waves). These are also the two selected cases for our study here.

As in the FitzHugh-Nagumo system, we obtain the $\mathrm{CV}$ restitution curves by continuation using AUTO, and use a solution at its lower branch as the critical pulse; see fig. 16. The corresponding propagation speeds are given in Table III.

\section{B. Linear theory}

Fig. 17 and Table III illustrate other ingredients required for the semi-analytical prediction of the critical curves for the two selected cases. As for the FitzHughNagumo system, these are found by the straightforward

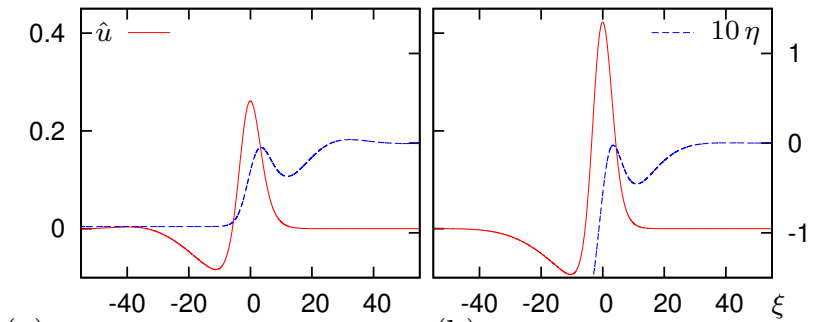

(a) (b)

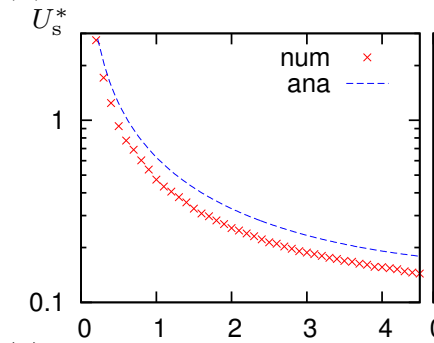

(c)

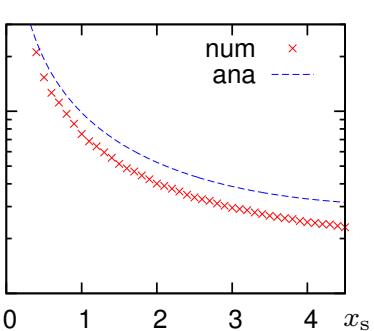

(d)

FIG. 15: (Color online) Results of linearized FitzHughNagumo theory for $(\mathrm{a}, \mathrm{c}) \beta=0.05$ and $(\mathrm{b}, \mathrm{d}) \beta=0.13$. $(\mathrm{a}, \mathrm{b})$ : The pre-compatibility function $\eta(\xi)$, used to compute the theoretical critical curve. The $\hat{u}(\xi)$ component of the critical solution $\hat{\mathbf{u}}$ is also shown for positioning purposes. (c,d): Comparison of the theoretical critical curves obtained in the linear approximation, and the critical curves obtained by direct numerical simulations.

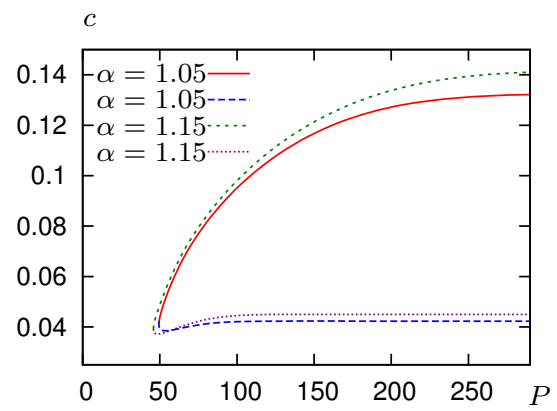

FIG. 16: (Color online) CV restitution curves for the BR model for two selected values of the model parameter. Stable (upper) and unstable (lower) branches are shown by different line types.

marching method and then verified by Arnoldi iterations. We use discretization $\Delta_{x}=0.03, \Delta_{t}=4 \Delta_{x}^{2} / 9$, $\xi \in[-L, L], L=90$. Again, we note that numerically found values of $\left|\lambda_{2}\right|$ are small.

The pre-compatibility functions $\eta(\xi)$ (see fig. 18(a)) are this time nearly unimodal with a prominent maxi-

\begin{tabular}{|c|c|c|c|}
\hline$\alpha$ & $c$ & $\lambda_{1}$ & $\lambda_{2}$ \\
\hline 1.05 & 0.04232 & 0.01578 & $\pm 2 \cdot 10^{-8}$ \\
\hline 1.15 & 0.04497 & 0.01515 & $\pm 1 \cdot 10^{-8}$ \\
\hline
\end{tabular}

TABLE III: Nonlinear and linear eigenvalues for the modified Beeler-Reuter model. 

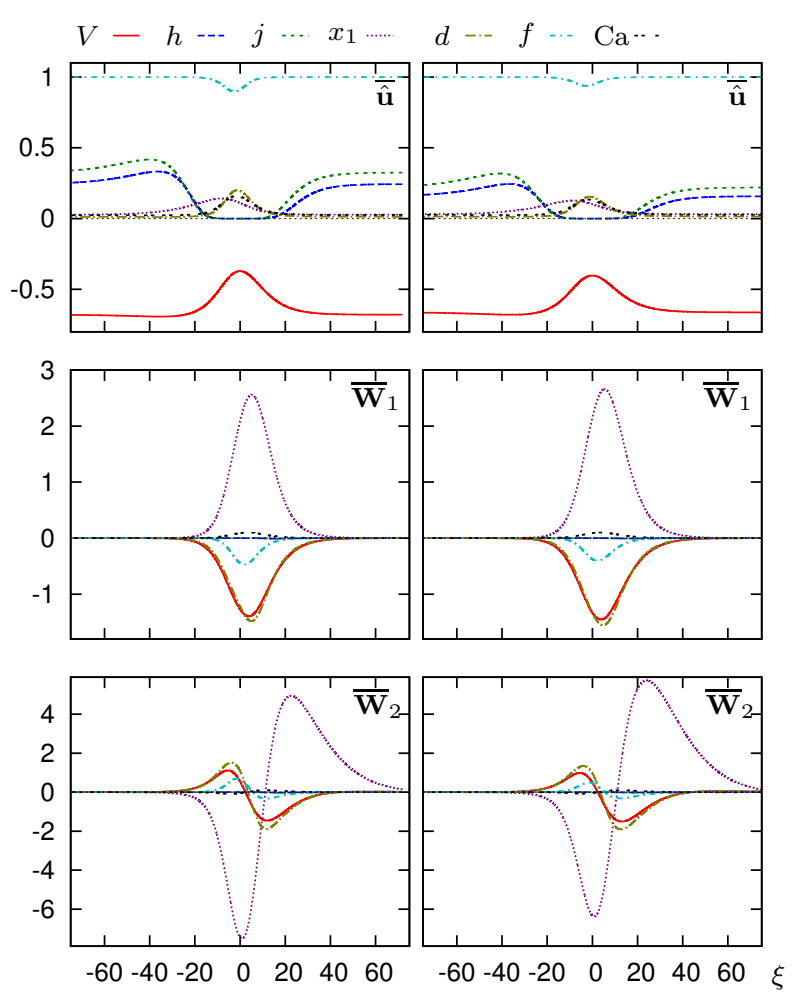

(a)

(b)

FIG. 17: (Color online) BR theory ingredients for (a) $\alpha=0.105$ and (b) $\alpha=0.115$. Shown are components of scaled vector functions, indicated in top right corner of each panel, where $\ldots \overline{\hat{\mathbf{u}}}=\mathbf{S} \hat{\mathbf{u}}, \overline{\mathbf{W}}_{j}=10^{4} \mathbf{S}^{-1} \mathbf{W}_{j}$, and $\mathbf{S}=\operatorname{diag}\left(10^{-2}, 1,1,1,1,1,10^{5}\right)$. The space coordinate is chosen so that $\xi=0$ at the maximum of $\hat{V}$. Correspondence of lines with components is according to the legends at the top.

mum near the front or the peak of the critical nucleus. Again, despite apparent similarity of the eigenfunctions in fig. 17 between the two cases, the shape of the $\eta(\xi)$ graphs in fig. $18(\mathrm{a}, \mathrm{b})$ is considerably different, and the resulting theoretical critical curves, shown in fig. 18 (c,d) are much better for $\alpha=0.105$ than for $\alpha=0.115$. This time, $\lambda_{1}$ is nearly the same in both cases.

\section{DISCUSSION}

In this paper, we have substantially extended the method proposed in 25] for an analytical description of the threshold curves that separate the basins of attraction of propagating wave solutions and of decaying solutions of certain reaction-diffusion models of spatiallyextended excitable media. The method is extended in two ways. Firstly, it is generalized to address a wider class of excitable systems, such as:

- multicomponent reaction-diffusion systems;

- systems with non-self-adjoint linearized operators;

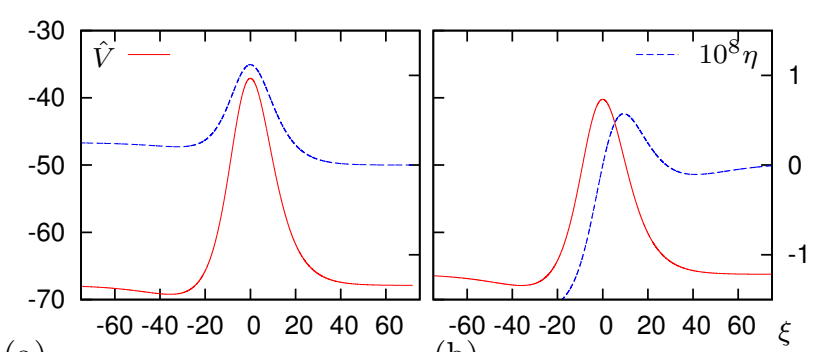

(a)

(b)

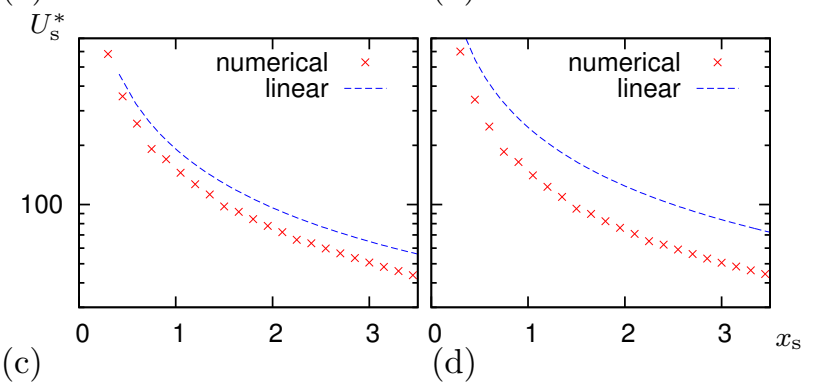

FIG. 18: (Color online) Results of BR theory for $(\mathrm{a}, \mathrm{c})$ $\alpha=0.105$ and (b,d) $\alpha=0.115$. (a,b): The pre-compatibility function $\eta(\xi)$, used to compute the theoretical critical curve (dashed lines, right ordinate axes). The $\hat{V}(\xi)$ component of the critical solution $\hat{\mathbf{u}}$ is also shown for positionining purposes (solid lines, left ordinate axes). (c,d): Comparison of the theoretical critical curves obtained in the linear approximation, and the critical curves obtained by direct numerical simulations.

- in particular, systems with moving critical solutions (critical fronts and critical pulses).

Secondly, the method is extended from being a linear approximation to being

- a quadratic approximation

of the stable manifold of the critical nucleus solution, resulting in some cases in a significant increase in accuracy.

The essential ingredients of the theory are the critical solution itself, and the eigenfunctions of the corresponding linearized operator. For the linear approximation in the critical nucleus case, we need the leading left (adjoint) eigenfunction; in the moving critical solution case, we need two leading left eigenfunctions; and for the quadratic approximations we need as many eigenvalues and left and right eigenfunctions as possible to achieve better accuracy. Of course, closed analytical formulas for these ingredients can only be obtained in exceptional cases, and in a more typical situation a "hybrid" approach is required, where these ingredients are obtained numerically. Still, we believe that this approach offers advantage over the determination of the excitation threshold by direct numerical simulations, both in terms of insight and computational cost.

It is still an open question, why in some cases our method works better than in others. A partial answer to that question is offered by the quadratic approximation: the linear approximation performs better when the 
corrections offered by the quadratic approximation are small. This seems to work for scalar equation with stationary critical solutions (critical nucleus). However, in this paper we have not investigated the quadratic approximation for the cases of moving critical solutions, that is, critical fronts and critical pulses. Here one has to bear in mind that the theory was presented here under the assumption that the spectrum is real, whereas in the non-self-adjoint case it does not have to be. So, this question remains an interesting direction for future research. Progress in that direction may help to understand when the linear approximation works better in such cases.

Another direction for future research is the possible extension of the theory to different initiation protocols, most notably, to the case of strength-duration curves for stimulation of an excitable cable by a stimulus localized in space and extended in time. In 25] we have shown that in the scalar case, the linearized theory readily gives the classical Lapique-Blair-Hill exponential rheobase-chronaxie expression. It is known that in realistic excitable systems, this formula does not always work well, and it is likely that the more complicated expression coming out of our theory based on moving critical solutions would perform better.

An obvious extension of our approach that is required for many applications is the extension to two and three spatial dimensions.

It is also of interest to investigate whether the proposed semi-analytical approach to ignition of excitation waves can be adapted to address the reverse problem of establishing conditions for decay (block) of an already propagating excitation wave. This question is of particular importance in practical situations, for instance for wild fire extinction, cardiac defibrillation and others. Some crude criteria for block of excitation can be established from asymptotic considerations of conditions when propagating wave solutions cease to exist, e.g. 51, however extention of the approach presented in this paper may offer more refined criteria. Propagating waves do not have the shape of rectangular pulses, as typical stimuli do, and decay from a general wave form must be considered in greater detail. An extra feature in two and three dimensions is the possibility of "wave breaks" which is a situation distinct from a complete decay and which is of particular relevance for cardiac arrhythmias.

Finally, we note that the problem of initiation of waves is of importance in all excitable systems, not just in cardiology. The theory presented here is likely to face new challenges in new applications. For instance, combustion waves sometimes can propagate in oscillatory manner, i.e. as relative periodic orbits [52, which makes it plausible that the critical solution there also is a relative periodic orbit, and the transition to turbulence in shear flows, although exhibiting features of excitability, is in terms of models beyond reaction-diffusion even in the simplest phenomenological description [10.

\section{Acknowledgements}

Research that led to this publication was supported in part by EPSRC grants GR/S75314 and EP/I029664 and London Mathematical Society (UK), and by McArthur Foundation (Nigeria). VNB is grateful to A.N. Gorban and D. Barkley for helpful discussions about a number of technical issues.

\section{Appendices}

\section{Appendix A: On "frozen nuclei" in the McKean equation}

Direct numerical simulations in this model are more difficult because in the standard finite-difference discretization, the critical nucleus solution, defined as an even, spatially nontrivial, stationary solution of the discretized equation, may not be unique and is stable, whereas in the differential equation it is unique and unstable. This phenomenon is akin to "propagation block" or "propagation failure" observed in discretized equation of the ZFK and McKean type and discussed e.g. in 43, 53, 54, with the exception that here we are dealing with even solutions, which correspond to spatially localized solutions when extended to the whole line, as opposed to the trigger front solutions which are traditionally the object of interest in the context of propagation block. Keener's 43. result is about a generic system with smooth right-hand sides, and it predicts "frozen solutions" for sufficiently large discretization steps. As we shall see, for the McKean model with its discontinous right hand side, the situation is different in that the frozen solutions, at least formally, exist for all discretization steps.

For the McKean model, the "discrete critical nucleus" solutions can be studied analytically. For the regular grid discretization,

$$
u_{j}=u\left(x_{j}\right), \quad x_{j}=j \Delta_{x}, \quad j \in \mathbb{Z}, \quad u_{-j} \equiv u_{j},
$$

we have

$$
\frac{\mathrm{d} u_{j}}{\mathrm{~d} t}=\frac{u_{j-1}-2 u_{j}+u_{j+1}}{\Delta_{x}^{2}}-u_{j}+\mathrm{H}\left(u_{j}-a\right) .
$$

We use notation $\overline{A, B}$ for the set of all integers $j$ such that $j \geq A$ and $j \leq B$, that is, $\overline{A, B} \triangleq[A, B] \cap \mathbb{Z}$. For the critical nucleus solution, we expect that there exists an integer $m$ such that $u_{j}>a$ for $j \in \overline{-m, m}$ and $u_{j}<a$ otherwise. We ignore the exceptional case when $u_{j}=a$ exactly for some $j$ as it is not interesting in practice. Let $u_{j}=v_{j}+\mathrm{H}\left(u_{j}-a\right)$. Then, separately on each of the intervals $j \in \overline{-\infty,-m-1}, j \in \overline{-m, m}$ and $j \in \overline{m+1, \infty}$, we have

$$
v_{j-1}-2 v_{j}+v_{j+1}-\Delta_{x}^{2} v_{j}=0 .
$$


For $v_{j} \propto \sigma^{j}$, this gives

$$
\sigma^{2}-\left(2+\Delta_{x}^{2}\right) \sigma+1=0
$$

and so $\sigma=\mu$ or $\sigma=1 / \mu$, where

$$
\mu \triangleq 1+\Delta_{x}^{2} / 2+\Delta_{x} \sqrt{1+\Delta_{x}^{2} / 4}>1
$$

We note that equation (A1) applied for $j \in \overline{-m, m}$ in fact involves $v_{j}$ for $j \in \overline{-m-1, m+1}$, and the same equation applied for $j \in \overline{m+1, \infty}$ describes $v_{j}$ for $j \in \overline{m, \infty}$, so the non-overlapping sub-intervals of the equation create overlapping sub-intervals in the piecewise described solutions.

Considering A1 for $j \in \overline{m+1, \infty}$ with account of the boundary condition $\lim _{j \rightarrow \infty} u_{j}=0$, we have

$$
u_{j}=v_{j}=A \mu^{-j}, \quad j \in \overline{m, \infty}
$$

for some constant $A$. Further, considering (A1) for $|j| \leq$ $m$, we get the even solution in the form

$$
u_{j}=1+v_{j}=1+B\left(\mu^{j}+\mu^{-j}\right), \quad j \in \overline{-m-1, m+1}
$$

for some constant $B$. The matching condition to determine $A$ and $B$ is that the two solutions should coincide at the overlap points, $j=m$ and $j=m+1$. This gives

$$
A=\frac{\left(\mu^{2 m+1}-1\right)}{\mu^{m}(\mu+1)}, \quad B=-\frac{1}{\mu^{m}(\mu+1)},
$$

and the nontrivial time-independent solution $u_{j}(t) \equiv \hat{u}_{j}$ in the form

$$
\hat{u}_{j}= \begin{cases}1-\frac{\mu^{j}+\mu^{-j}}{\mu^{m}(\mu+1)}, & j \in \overline{0, m+1}, \\ \frac{\left(\mu^{2 m+1}-1\right) \mu^{-j}}{\mu^{m}(\mu+1)}, & j \in \overline{m, \infty} .\end{cases}
$$

This result is valid under the assumption that

$$
\begin{aligned}
& a \in\left(\hat{u}_{m+1}, \hat{u}_{m}\right)=\left(\frac{\mu^{m}-\mu^{-m-1}}{\mu^{m+1}+\mu^{m}}, \frac{\mu^{m+1}-\mu^{-m}}{\mu^{m+1}+\mu^{m}}\right) \\
& =\left(\underline{a}_{m}, \bar{a}_{m}\right) .
\end{aligned}
$$

This gives a range of possible values of $a$ for a given $m$. So, at a fixed $\Delta_{x}$, the dependence of the solution on parameter $a$ is discontinuous (piecewise constant), and there is a possibility that at some combinations of $\Delta_{x}$ and $a$, there could be more than one solution. Indeed, this possibility is realized if the intervals $\left(\underline{a}_{m}, \bar{a}_{m}\right)$ for consecutive values of $m$ overlap, that is,

$$
\underline{a}_{m+1}<\bar{a}_{m}
$$

which is the case whenever

$$
m>m_{1} \triangleq \frac{\log \left(\mu^{2}+\mu+1\right)}{2 \log \mu}-\frac{3}{2} .
$$

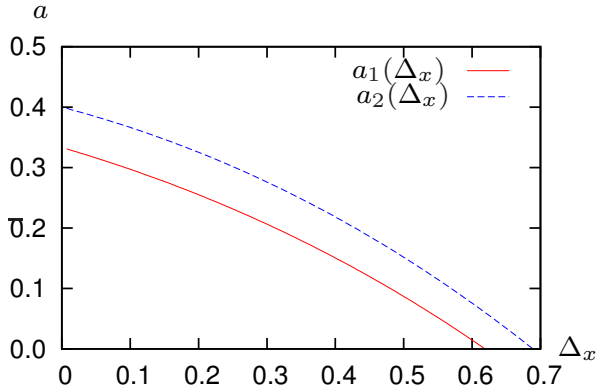

FIG. 19: (Color online) Non-uniqueness of the discrete critical nucleus solutions is observed for some $a>a_{1}\left(\Delta_{x}\right)$, and for all $a>a_{2}\left(\Delta_{x}\right)$.

Considering that the discrete solution $\sqrt{\mathrm{A} 3}$ approximates the exact critical nucleus solution $(73)$, we have the corresponding matching point coordinate

$$
x_{*}>x_{* 1} \approx \Delta_{x} m_{1},
$$

and then from 74

$$
a>a_{1} \approx \frac{1}{2}\left(1-\mathrm{e}^{-2 x_{* 1}}\right) .
$$

Equations (A2), (A4), (A5) and (A6) define $a_{1}$ as a function of $\Delta_{x}$, such that for $a>a_{1}\left(\Delta_{x}\right)$ there can be more than one discrete solution corresponding to the same $a$. The graph of this function is shown in fig. 19. Similarly, by solving the inequality

$$
\underline{a}_{m+2}<\bar{a}_{m}
$$

we get the function $a_{2}\left(\Delta_{x}\right)$ such that for $a>a_{2}\left(\Delta_{x}\right)$ the non-uniqueness of the discrete solutions is not only possible, but is guaranteed (we omit the straightforward but bulky derivation). The graph of this function is also shown in fig. 19 .

However the question of stability of the discrete critical nucleus solution is more important, even if this solution is unique. For $u_{j}(t)=\hat{u}_{j}+v_{j}(t)$, the linearized system is

$$
\frac{\mathrm{d} v_{j}}{\mathrm{~d} t}=\frac{v_{j-1}-2 v_{j}+v_{j+1}}{\Delta_{x}^{2}}-v_{j}, \quad j \in \mathbb{Z},
$$

"almost certainly", again with the exception of the cases when one of $\hat{u}_{j}=a$ exactly. The spectrum of the system A7 in $\ell^{2}(\mathbb{Z})$ is $\left[-1-4 / \Delta_{x}{ }^{2},-1\right]$, with eigenpairs

$$
W_{j}=\exp (i \kappa j), \quad \lambda=-1-2(1-\cos \kappa) / \Delta_{x}{ }^{2}, \quad \kappa \in \mathbb{R} .
$$

So the discretized critical nuclei are almost surely asymptotically stable in the linear approximation (remember that we did not analyse the exceptional cases when $u_{j}=a$ exactly at some $j$ ). As a result, in our simulations at initiation parameters close to the threshold, we find distinct "frozen critical nucleus" solutions, so that the critical curve between ignition and failure becomes in fact a "critical band". "Convergence" of the discretized 


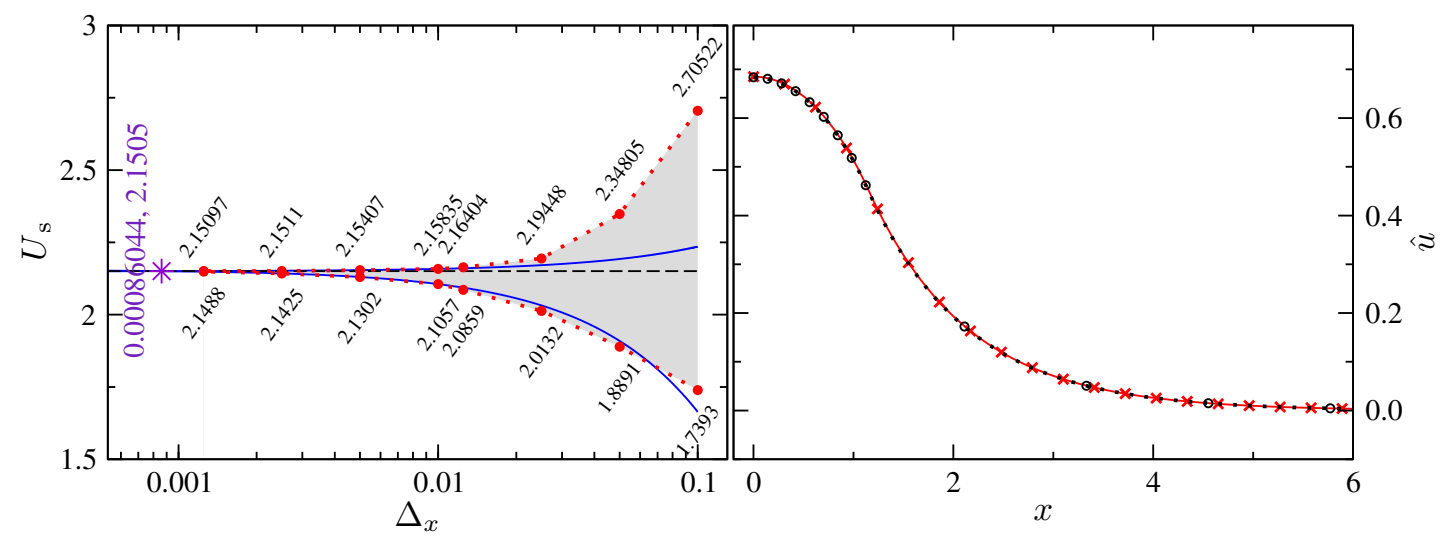

FIG. 20: (Color online) The region where "frozen nuclei" solutions occur in direct numerical simulations of the McKean model $\sqrt{72}$ for $a=0.45$ and $x_{\mathrm{s}}=0.5$ and with variation of the space step $\Delta_{x}$ is located between the two dotted red lines with annotated data points and is shaded in gray. The thin solid blue lines are lines of linear extrapolation the intersection of which indicated by the violet star and annotated is taken as the boundary between decay and ignition. (b) Comparison between the "frozen" solution obtained by direct numerical simulation at $a=0.45, x_{\mathrm{s}}=0.5, U_{\mathrm{s}}=2.13, \Delta_{x}=0.01$ and the known exact analytical expression for the critical nucleus $(73$ at $a=0.45$.

system to the continuous system, as far as the initiation problem is concerned, is manifested by reduction of the basin of attraction of the critical nucleus solutions with decreasing discretization steps, as is also evident in fig. 19. This convergence is illustrated in fig. 20.

\section{Appendix B: On linearization of discontinuous right-hand sides}

Rinzel and Keller [42] obtained operator (75) by formally differentiating the nonlinearity of $(72)$, apparenly having in mind a calculation like

$$
\begin{aligned}
& \frac{\partial f(u)}{\partial u}=\frac{\partial f(u(x))}{\partial x} / \frac{\partial u(x)}{\partial x} \\
& =\left(\frac{\partial u(x)}{\partial x}\right)^{-1} \frac{\partial}{\partial x}\left(-u+\mathrm{H}\left(x_{*}-x\right)\right) \\
& =\frac{1}{u^{\prime}(x)}\left(-u^{\prime}(x)-\delta\left(x_{*}-x\right)\right) \\
& =-1-\frac{1}{u^{\prime}\left(x_{*}\right)} \delta\left(x-x_{*}\right) .
\end{aligned}
$$

This might look paradoxical, as the linearization procedure in its traditional understanding is based on the assumption of smallness of the increments, whereas discontinuity of the reaction term means that in some circumstances increments of those terms are not small when the increments of the arguments vanish, which is an apparent contradiction. Nevertheless, this procedure can be mathematically justified.

Consider, as a simple example, a scalar reactiondiffusion equation for some field $u(x, t)$, with a single discontinuity at $u=0$ :

$$
u_{t}=f_{1}(u)+\left(f_{2}(u)-f_{1}(u)\right) \mathrm{H}(u)+D u_{x x} .
$$

Let us consider a one-parametric family of solutions, $u=U(x, t ; p)$, which is continuous and piecewisedifferentiable in $x, t$ and $p$. We are seeking a differential equation for $v=\partial U / \partial p$, that is, the linearized equation, or equation in variations. Since the function $v$ is expected to be discontinuous, its differential equation will contain singular terms, i.e. it should be understood in terms of generalized functions (distributions).

Suppose that our solution is monotonically decreasing in $x$ in some region of the $(x, t)$ plane containing the curve $x=s(t)$ at which $U$ changes sign (the opposite case is considered in a similar way). Then (B1) can be rewritten as

$$
\begin{aligned}
& U_{t}=f_{1}(U)+\left(f_{2}(U)-f_{1}(U)\right) \mathrm{H}(s(t)-x)+D U_{x x} \\
& U(s(t), t ; p)=0
\end{aligned}
$$

By differentiating this with respect to $p$, we have

$$
\begin{aligned}
& U_{t p}=f_{1}{ }^{\prime}(U) U_{p}+\left(f_{2}{ }^{\prime}(U)-f_{1}{ }^{\prime}(U)\right) \mathrm{H}(s(t)-x) U_{p} \\
& \quad+D U_{x x p}+\left(f_{2}(0)-f_{1}(0)\right) \delta(s(t)-x) s_{p}, \\
& U_{p}(s(t), t ; p)+U_{x}(s(t), t ; p) s_{p}=0 .
\end{aligned}
$$

By excluding $s_{p}$ from this system and setting $U_{p}=v$, we have

$$
\begin{aligned}
& v_{t}=f_{1}{ }^{\prime}(U) v+\left(f_{2}{ }^{\prime}(U)-f_{1}{ }^{\prime}(U)\right) \mathrm{H}(s(t)-x) v \\
& +D v_{x x}+\frac{f_{2}(0)-f_{1}(0)}{U_{x}(s, t ; p)} \delta(s(t)-x) v
\end{aligned}
$$

which is precisely the linearized equation which would be obtained by formal differentiation of the right-hand side of (B1), and subsequent replacement of $\delta(U)$ by $\delta(s-x)$ using the chain rule. 


\section{Appendix C: Finite element discretization for the McKean model}

For the McKean model, we needed to solve initial-value problems both for the nonlinear equation,

$$
\frac{\partial u}{\partial t}=\frac{\partial^{2} u}{\partial x^{2}}-u+\mathrm{H}(u-a)
$$

and its linearization,

$$
\frac{\partial v}{\partial t}=\frac{\partial^{2} v}{\partial x^{2}}-v+\delta(u-a) v .
$$

These are equivalent (for $u$ decreasing in $x$ ) to

$$
\begin{aligned}
& \frac{\partial u}{\partial t}=\frac{\partial^{2} u}{\partial x^{2}}-u+\mathrm{H}\left(x_{*}-x\right) \\
& \frac{\partial v}{\partial t}=\frac{\partial^{2} v}{\partial x^{2}}-v+\frac{1}{u^{\prime}\left(x_{*}\right)} \delta\left(x-x_{*}\right) v
\end{aligned}
$$

where $x_{*}=x_{*}(t)$ is defined by

$$
u\left(x_{*}(t), t\right)=a .
$$

Both cases required finite-element treatment, and we present the details for both cases together, by writing both as

$$
\frac{\partial w}{\partial t}=\frac{\partial^{2} w}{\partial x^{2}}+f(w, x)
$$

where $w=u, f=-u+\mathrm{H}(u-a)=-u+\mathrm{H}\left(x_{*}-x\right)$ in one case, and $w=v, f=-v+\delta(u-a) v=-v+$ $\left(1 / u^{\prime}\left(x_{*}\right)\right) \delta\left(x-x_{*}\right) v$ in the other case.

The finite element method (see e.g. 55]) is based on a weak formulation of the problem, which requires that

$$
\int_{0}^{L} \Phi(x)\left\{\frac{\partial w}{\partial t}-\frac{\partial^{2} w}{\partial x^{2}}-f(w, x)\right\} \mathrm{d} x=0
$$

for any "test function" $\Phi(x)$. If the variety of the available test functions is broad enough then the weak formulation is equivalent to the original equation. After integration by parts and taking into account the Neumann boundary conditions for $w$, the weak formulation can be formally re-written as

$$
\int_{0}^{L}\left[\Phi(x)\left(\frac{\partial w}{\partial t}-f(w, x)\right)+\frac{\partial \Phi}{\partial x} \frac{\partial w}{\partial x}\right] \mathrm{d} x=0 .
$$

The difference is, of course, that whereas the original formulation requires that $w$ is twice differentiable in $x$, the weak formulation (C1) uses only first derivatives of $w$, and can be applied as long as the test functions $\Phi$ are once differentiable.

The standard finite element method is the Galerkin method applied to (C1). It uses a set of linearly independent functions, $\Phi_{j}(x), j=0, \ldots, N$, called the finite elements, as the test functions, and seeks the approximation of the solution in the span of this same set:

$$
w(x, t) \approx \check{w}(x, t)=\sum_{j=0}^{N} \check{w}_{j}(t) \Phi_{j}(x) .
$$

Substitution of (C2) into $\mathrm{C} 1$ for $\Phi=\Phi_{i}, i=0, \ldots, N$, leads to the system of equations

$$
\sum_{j=0}^{N} A_{i, j} \frac{\mathrm{d} \check{w}_{j}}{\mathrm{~d} t}+\sum_{j=0}^{N} B_{i, j} \check{w}_{j}=C_{i}\left(\check{w}_{j}\right), \quad i=0, \ldots, N
$$

or in the vector form, for $\check{\mathbf{w}}(t)=\left(\check{w}_{j}\right)$,

$$
\mathbf{A} \frac{\mathrm{d} \check{\mathbf{w}}}{\mathrm{d} t}+\mathbf{B} \check{\mathbf{w}}=\mathbf{C}
$$

where the coefficients are given by

$$
\begin{aligned}
& A_{i, j}=\int_{0}^{L} \Phi_{i}(x) \Phi_{j}(x) \mathrm{d} x \\
& B_{i, j}=\int_{0}^{L} \Phi_{i}^{\prime}(x) \Phi_{j}^{\prime}(x) \mathrm{d} x \\
& C_{i}\left(\check{w}_{j}\right)=\int_{0}^{L} \Phi_{i}(x) f\left(\sum_{j=0}^{N} \check{w}_{j} \Phi_{j}(x), x\right) \mathrm{d} x .
\end{aligned}
$$

We use a simple and popular choice of the test functions, the piecewise linear tent functions:

$$
\Phi_{i}(x)= \begin{cases}\left(x-x_{i-1}\right) / \Delta_{x}, & x \in\left[x_{i-1}, x_{i}\right], \\ \left(x_{i+1}-x\right) / \Delta_{x}, & x \in\left[x_{i}, x_{i+1}\right], \\ 0, & \text { otherwise }\end{cases}
$$

for a regular grid of $\left(x_{i}\right)$,

$$
x_{i}=i \Delta_{x}, \quad i=0, \ldots, N, \quad \Delta_{x}=L / N .
$$

Obviously, in this case $\Phi_{j}\left(x_{i}\right)=\delta_{i, j}$ and therefore $\check{w}\left(x_{i}\right)=\check{w}_{i}$. For these test functions, $\mathrm{C} 4$ give the mass matrix $\mathbf{A}=\left(A_{i, j}\right)$ as

$$
\mathbf{A}=\frac{\Delta_{x}}{6}\left(\begin{array}{ccccc}
2 & 1 & 0 & \cdots & 0 \\
1 & 4 & 1 & & \vdots \\
0 & \ddots & \ddots & \ddots & 0 \\
\vdots & & 1 & 4 & 1 \\
0 & \cdots & 0 & 1 & 2
\end{array}\right)
$$

the stiffness matrix $\mathbf{B}=\left(B_{i, j}\right)$ as

$$
\mathbf{B}=\frac{1}{\Delta_{x}}\left(\begin{array}{ccccc}
1 & -1 & 0 & \cdots & 0 \\
-1 & 2 & -1 & & \vdots \\
0 & \ddots & \ddots & \ddots & 0 \\
\vdots & & -1 & 2 & -1 \\
0 & \cdots & 0 & -1 & 1
\end{array}\right)
$$


and the load vector $\mathbf{C}=\left(C_{i}\right)$ as

$$
\mathbf{C}(\check{\mathbf{w}})=-\mathbf{A} \check{\mathbf{w}}+\mathbf{F},
$$

where $\mathbf{F}=\left(F_{i}\right)$ and differs for the nonlinear problem and for the linearized problem.

For the nonlinear problem, $\check{\mathbf{w}}=\check{\mathbf{u}}$, we get $\mathbf{F}=\mathbf{F}^{(1)}+$ $\mathbf{F}^{(2)}$, where

$$
F_{i}^{(1)}=\frac{1}{2 \Delta_{x}} \begin{cases}\Delta_{x}{ }^{2}, & \check{u}_{i-1}>a, \check{u}_{i}>a, \\ \left(x_{*}-x_{i-1}\right)^{2}, & \check{u}_{i-1}>a, \check{u}_{i}<a, \\ \Delta_{x}{ }^{2}-\left(x_{*}-x_{i-1}\right)^{2}, & \check{u}_{i-1}<a, \check{u}_{i}>a \\ 0, & \text { otherwise, }\end{cases}
$$

and

$$
F_{i}^{(2)}=\frac{1}{2 \Delta_{x}} \begin{cases}\Delta_{x}{ }^{2}, & \check{u}_{i+1}>a, \check{u}_{i}>a, \\ \left(x_{*}-x_{i+1}\right)^{2}, & \check{u}_{i+1}>a, \check{u}_{i}<a, \\ \Delta_{x}{ }^{2}-\left(x_{*}-x_{i+1}\right)^{2}, & \check{u}_{i+1}<a, \check{u}_{i}>a, \\ 0, & \text { otherwise, }\end{cases}
$$

for $i=1, \ldots, N-1$, and

$$
F_{0}=\frac{1}{4 \Delta_{x}} \begin{cases}2 \Delta_{x}{ }^{2}, & \check{u}_{0}>a, \check{u}_{1}>a, \\ \left(x_{*}-x_{-1}\right)^{2} & \\ +\left(x_{1}-x_{*}\right)^{2}, & \check{u}_{0}<a, \check{u}_{1}>a, \\ 2 \Delta_{x}{ }^{2}-\left(x_{*}-x_{-1}\right)^{2} & \\ -\left(x_{1}-x_{*}\right)^{2}, & \check{u}_{0}>a, \check{u}_{1}<a, \\ 0, & \text { otherwise, }\end{cases}
$$

and

$$
F_{N}=\frac{1}{4 \Delta_{x}} \begin{cases}2 \Delta_{x}^{2}, & \check{u}_{N-1}>a, \check{u}_{N}>a, \\ \left(x_{*}-x_{N+1}\right)^{2} & \\ +\left(x_{N-1}-x_{*}\right)^{2}, & \check{u}_{N-1}>a, \check{u}_{N}<a, \\ 2 \Delta_{x}^{2}-\left(x_{*}-x_{N+1}\right)^{2} & \\ -\left(x_{N-1}-x_{*}\right)^{2}, & \check{u}_{N-1}<a, \check{u}_{N}>a, \\ 0, & \text { otherwise. }\end{cases}
$$

for the boundary points. In these formulas, $x_{*}$ is the point such that $\breve{u}\left(x_{*}, t\right)=a$ by linear interpolation, i.e. for $m$ such that $\left(\check{u}_{m}-a\right)\left(\check{u}_{m+1}-a\right) \leq 0$, we have $x_{*}=$ $\left(\left(\check{u}_{m+1}-a\right) x_{m}+\left(a-\check{u}_{m}\right) x_{m+1}\right) /\left(\check{u}_{m+1}-\check{u}_{m}\right)$, and the definition (C6) is extended to $i=-1$ and $i=N+1$.
For the linear problem, $\check{\mathbf{w}}=\check{\mathbf{v}}$, we get

$$
\begin{aligned}
& F_{m}=\frac{1}{a \Delta_{x}^{2}}\left[\left(x_{m+1}-x_{*}\right)^{2} \check{v}_{m}\right. \\
& \left.+\left(x_{m+1}-x_{*}\right)\left(x_{*}-x_{m}\right) \check{v}_{m+1}\right] \\
& F_{m+1}=\frac{1}{a \Delta_{x}^{2}}\left[\left(x_{m+1}-x_{*}\right)\left(x_{*}-x_{m}\right) \check{v}_{m}\right. \\
& \left.+\left(x_{*}=-x_{m}\right)^{2} \check{v}_{m+1}\right] \\
& F_{j}=0, \quad j \neq m, m+1,
\end{aligned}
$$

where $m$ and $x_{*}$ are defined based on the nonlinear solution $\check{\mathbf{u}}$ based on the same rule as above.

\section{Appendix D: The modified Beeler-Reuter model}

The model was proposed in [46. We use it in the following formulation: $\mathbf{f}:\left(V, h, j, x_{1}, d, f, \mathrm{Ca}\right)^{\top} \mapsto$ $\left(f_{V}, f_{h}, f_{j}, f_{x_{1}}, f_{d}, f_{f}, f_{\text {Ca }}\right)^{\top}$, where

$$
\begin{aligned}
f_{V}= & -I_{K_{1}}(V)-I_{x 1}\left(V, x_{1}\right) \\
& -I_{N a}(V, h, j)-I_{s}(V, d, f, \mathrm{Ca}), \\
f_{h}= & \alpha_{h}(V)(1-h)-\beta_{h}(V) h, \\
f_{j}= & \alpha_{j}(V)(1-j)-\beta_{j}(V) j, \\
f_{x_{1}}= & \alpha_{x 1}(V)\left(1-x_{1}\right)-\beta_{x 1}(V) x_{1}, \\
f_{d}= & \alpha_{d}(V)(1-d)-\beta_{d}(V) d, \\
f_{f}= & \alpha_{f}(V)(1-f)-\beta_{f}(V) f, \\
f_{\mathrm{Ca}}= & -10^{-7} I_{s}+0.07\left(10^{-7}-\mathrm{Ca}\right),
\end{aligned}
$$

where the transmembrane currents are defined by

$$
\begin{aligned}
& I_{K_{1}}(V)=0.35(0.3-\alpha) \overline{I_{K_{1}}}(V), \\
& \overline{I_{K_{1}}}(V)=\frac{4\left(\mathrm{e}^{0.04(V+85)}-1\right)}{\mathrm{e}^{0.08(V+53)}+\mathrm{e}^{0.04(V+53)}} \\
& \quad+\frac{0.2(V+23)}{1-\mathrm{e}^{-0.04(V+23)}}, \\
& I_{x 1}\left(V, x_{1}\right)=g_{i x}(V) x_{1}, \\
& g_{i x}(V)=0.8 \frac{\mathrm{e}^{0.04(V+77)}-1}{\mathrm{e}^{0.04(V+35)}}, \\
& I_{N a}(V, h, j)=\left(g_{N a}(m(V))^{3} h j+g_{N a_{c}}\right)\left(V-E_{N a}\right), \\
& I_{s}(V, d, f, \mathrm{Ca})=g_{s} d f\left(V-E_{s}(\mathrm{Ca})\right),
\end{aligned}
$$

the $m$-gate is assumed in quasi-stationary state,

$$
m(V)=\alpha_{m}(V) /\left(\alpha_{m}(V)+\beta_{m}(V)\right),
$$


the gate opening and closing rates are defined by

$$
\begin{aligned}
& \alpha_{x 1}(V)=\frac{0.0005 \mathrm{e}^{0.083(V+50)}}{\mathrm{e}^{0.057(V+50)}+1}, \\
& \beta_{x 1}(V)=\frac{0.0013 \mathrm{e}^{-0.06(V+20)}}{\mathrm{e}^{-0.04(V+20)}+1}, \\
& \alpha_{m}(V)=\frac{V+47}{1-\mathrm{e}^{-0.1(V+47)},} \\
& \beta_{m}(V)=40 \mathrm{e}^{-0.056(V+72)}, \\
& \alpha_{h}(V)=0.126 \mathrm{e}^{-0.25(V+77)}, \\
& \beta_{h}(V)=\frac{1.7}{\mathrm{e}^{-0.082(V+22.5)}+1}, \\
& \alpha_{j}(V)=\frac{0.055 \mathrm{e}^{-0.25(V+78)}}{\mathrm{e}^{-0.2(V+78)}+1}, \\
& \beta_{j}(V)=\frac{0.3}{\mathrm{e}^{-0.1(V+32)}+1}, \\
& \alpha_{d}(V)=\frac{0.095 \mathrm{e}^{-0.01(V-5)}}{\mathrm{e}^{-0.072(V-5)}+1},
\end{aligned}
$$

$$
\begin{aligned}
& \beta_{d}(V)=\frac{0.07 \mathrm{e}^{-0.017(V+44)}}{\mathrm{e}^{0.05(V+44)}+1}, \\
& \alpha_{f}(V)=\frac{0.012 \mathrm{e}^{-0.008(V+28)}}{\mathrm{e}^{0.15(V+28)}+1}, \\
& \beta_{f}(V)=\frac{0.0065 \mathrm{e}^{-0.02(V+30)}}{\mathrm{e}^{-0.2(V+30)}+1},
\end{aligned}
$$

and the calcium reversal potential is defined by the Nernst law,

$$
E_{s}(\mathrm{Ca})=-82.3-13.0287 \log (\mathrm{Ca}) .
$$

The parameters of the model are fixed at the values used in [47 50: $g_{N a}=0.24, g_{N a_{c}}=0.003, E_{N a}=50, g_{s}=$ 0.045 , and for $\alpha$ we consider two values $\alpha=0.105$ and $\alpha=0.115$.
[1] A. V. Gurevich and R. G. Mints. Self-heating in normal metals and superconductors. Rev. Mod. Phys., 59(4):941$1000,1987$.

[2] V. Krinsky and H. Swinney, editors. Wave and patterns in biological and chemical excitable media. NorthHolland, Amsterdam, 1991.

[3] S. K. Scott and K. Showalter. Simple and complex propagating reaction-diffusion fronts. J. Phys. Chem., 96(22):8702-8711, 1992.

[4] M. C. Cross and P. C. Hohenberg. Pattern formation outside of equilibrium. Rev. Mod. Phys., 65(3):851-1123, 1993.

[5] J. P. Keener and J. Sneyd. Mathematical Physiology. Springer, 1998.

[6] A. G. Merzhanov and E. N. Rumanov. Physics of reaction waves. Rev. Mod. Phys., 71(4):1173-1211, 1999.

[7] V. V. Bychkov and M. A. Liberman. Dynamics and stability of premixed flames. Physics Reports, 325:115-237, 2000.

[8] J. D. Murray. Mathematical Biology: I. An Introduction. Springer, 2002.

[9] S. Wieczorek, P. Ashwin, C. M. Luke, and P. M. Cox. Excitability in ramped systems: the compost-bomb instability. Proc. Roy. Soc. Lond. ser. A, 467(2129):12431269, 2011.

[10] D. Barkley. Modelling the transition to turbulence in shear flows. Journal of Physics: Conference series, 318:032001, 2011.

[11] D. P. Zipes and J. Jalife, editors. Cardiac electrophysiology: From cell to bedside. W B Saunders Co, 2004.

[12] A. A. Shah et al. The effects of heat exchange and fluid production on the ignition of a porous solid. Nonlinear Analysis: Real World Applications, 9(2):562-584, 2008.

[13] V. K. Dhir. Boiling heat transfer. Ann. Rev. Fluid Mech., 30:365-401, 1998.

[14] A. Pumir, V. V. Barelko, and E. V. Buryak. Control of the boiling crisis: analysis of a model system. Eur. Phys.
J. $B, 60: 1-8,2007$

[15] L. Lapicque. Recherches quantitative sur l'excitation electriquer des nerfs traitée comme une polarisation. $J$ Physiol (Paris), 1907.

[16] H. A. Blair. On the intensity-time relationship for simulation by electric current. J Gen Physiol, 15:709-729, 731-755, 1932.

[17] A. V. Hill. Excitation and accommodation in nerve. Proc $R$ Soc Lond (Biol), 119:305-355, 1936.

[18] W. A. H. Rushton. Initiation of the propagated disturbance. Proc. Roy. Soc. Lond. ser. B, 124:210-243, 1937.

[19] D. Noble. The relation of Rushton's 'liminal length' for excitation to the resting and active conductances of excitable cells. J. Physiol., 226:573-591, 1972.

[20] H. P. McKean and V. Moll. A threshold for a caricature of the nerve equation. Bull. AMS, 12(2):255-259, 1985.

[21] G. Flores. The stable manifold of the standing wave of the Nagumo equation. J. Differential Equations, 80:306314, 1989.

[22] J. C. Neu, R. S. Preissig, and W. Krassowska. Initiation of propagation in a one-dimensional excitable medium. Physica D, 102:285-299, 1997.

[23] S. Jacquir, S. Binczak, J. P. Gauthier, and J. M. Bilbault. Emergence of travelling waves in smooth nerve fibres. Discrete and Continuous Dynamical Systems series $S, 1(2): 263-272,2008$.

[24] V. Moll and S. I. Rosencrans. Calculation of the threshold surface for nerve equations. SIAM J. Appl. Math., 50(5):1419-1441, 1990.

[25] I. Idris and V. N. Biktashev. Analytical approach to initiation of propagating fronts. Phys. Rev. Lett., 101:244101, 2008.

[26] G. Flores. Stability analysis for the slow travelling pulse of the FitzHugh-Nagumo system. SIAM J. Math. Anal., 22(2):392-399, 1991.

[27] I. Idris and V. N. Biktashev. Critical fronts in initiation of excitation waves. Phys. Rev. E, 76(2):021906, 2007. 
[28] V. N. Biktashev and A. V. Holden. Deterministic Brownian motion in the hypermeander of spiral waves. Physica D, 116(3-4):342-354, 1998.

[29] V. N. Biktashev, A. V. Holden, and E. V. Nikolaev. Spiral wave meander and symmetry of the plane. Int. J. of Bifurcation and Chaos, 6(12A):2433-2440, 1996.

[30] A. J. Foulkes and V. N. Biktashev. Riding a spiral wave: Numerical simulation of spiral waves in a co-moving frame of reference. Phys. Rev. E, 81(4):046702, 2010.

[31] Y. Kuramoto. Instability and turbulence of wavefronts in reaction-diffusion systems. Prog. Theor. Phys., 63(6):1885-1903, 1980.

[32] V. N. Biktashev. Diffusion of autowaves. Evolution equation for slowly varying autowaves. Physica D, 40:83-90, 1989.

[33] H. Dierckx, O. Bernus, and H. Verschelde. Accurate eikonal-curvature relation for wave fronts in locally anisotropic reaction-diffusion systems. Phys. Rev. Lett., 107:108101, 2011.

[34] R. M. Beam and R. F. Warming. An implicit finite-difference algorithm for hyperbolic systems in conservation-law form. Journal of Computational Physics, 22(1):87-110, 1976.

[35] E. Doedel and J. P. Kernevez. AUTO, software for continuation and bifurcation problems in ordinary differential equations. California Institute of Technology, 1986.

[36] C. B. Moler. Numerical Computing with MATLAB: Revised Reprint. SIAM, 2008.

[37] R. J. Radke. A Matlab implementation of the implicitly restarted Arnoldi method for solving large-scale eigenvalue problems. Master's thesis, Rice University, 1996.

[38] P. C. Fife and J. B. McLeod. The approach of solutions of nonlinear diffusion equations to travelling front solutions. Arch. Rat. Mech. Anal., 65(4):335-361, 1977.

[39] Ya. B. Zel'dovich and D. A. Frank-Kamenetsky. Towards the theory of uniformly propagating flames. Doklady AN SSSR, 19:693-697, 1938.

[40] H. P. McKean, Jr. Nagumo's equation. Advances in Mathematics, 4(3):209-223, 1970.

[41] F. Schlögl. Chemical reaction models for non-equilibrium phase transitions. Z. Phys. A, 253(2):147-161, 1972.

[42] J. Rinzel and J. Keller. Traveling wave solutions of a nerve conduction equation. Biophys. J., 13(12):13131337, 1973.

[43] J. P. Keener. Propagation and its failure in coupled systems of discrete excitable cells. SIAM J. Appl. Math., 47(3):556-572, 1987.

[44] R. M. Corless, G. H. Gonnet, D. E. G. Hare, D. J. Jeffrey, and D. E. Knuth. On the Lambert W function. Advances in Computational Mathematics, 5:329-359, 1996.

[45] V. N. Biktashev. Dissipation of the excitation wavefronts. Phys. Rev. Lett., 89(16):168102, 2002.

[46] G. W. Beeler and H. Reuter. Reconstruction of the action potential of ventricular myocardial fibres. J. Physiol., 268:177-210, 1977.

[47] A. Arutunyan, A. Pumir, V. I. Krinsky, L. M. Swift, and N. Sarvazyan. Behavior of ectopic surface: effects of betaadrenergic stimulation and uncoupling. Am. J. Physiol.
- Heart and Circ. Physiol., 285(6):H2531-H2542, 2003

[48] A. Pumir, A. Arutunyan, V. Krinsky, and N. Sarvazyan. Genesis of ectopic waves: role of coupling, automaticity, and heterogeneity. Biophys. J., 89:2332-2349, 2005.

[49] V. N. Biktashev, A. Arutunyan, and N. A. Sarvazyan. Generation and escape of local waves from the boundary of uncoupled cardiac tissue. Biophys. J., 94:3726-3738, 2008.

[50] V. N. Biktashev, I. V. Biktasheva, and N. A. Sarvazyan. Evolution of spiral and scroll waves of excitation in a mathematical model of ischaemic border zone. PLoS ONE, 6(9):e24388, 2011.

[51] R. D. Simitev and V. N. Biktashev. Conditions for propagation and block of excitation in an asymptotic model of atrial tissue. Biophys. J., 90(7):2258-2269, 2006.

[52] K. J. Hughes, J. Brindley, and A. C. McIntosh. Initiation and propagation of combustion waves with competitive reactions and water evaporation. Proc. Roy. Soc. Lond. ser. A, 469:20130506, 2013.

[53] A. Hoffman and J. Mallet-Paret. Universality of crystallographic pinning. J. Dyn. Diff. Eq., 22:79-119, 2010.

[54] H. J. Hupkes, D. Pelinovsky, and B. Sandstede. Propagation failure in the discrete Nagumo equation. Proc. AMS, 139(10):3537-3551, 2011.

[55] J.N. Reddy. An Introduction to the Finite Element Method. McGraw-Hill series in mechanical engineering. McGraw-Hill, 2006.

[56] More precisely, since (4) is defined on half-line $x \in \mathbb{R}_{+}$ and the propagating wave solution (3) on the whole line $x \in \mathbb{R}$, the convergence should be understood in an appropriate sense, e.g. convergence in any finite interval fixed in a co-moving frame of reference.

[57] This assumption will, of course, have to be verified in each particular case.

[58] Actually, expressions given in both of these works contain typos.

[59] This fact has some implications for the spectrum of the problem: it depends on the functional space in which the linearized problem is considered. For instance in $L^{\infty}$ the zero is a double eigenvalue with a Jordan cell, the generalized eigenvector corresponding to $\partial_{\alpha} \hat{\mathbf{u}}$; however this mode does not belong in $L^{1}$ or $L^{2}$ so in those spaces the zero is a simple eigenvalue. This subtlety does not seem to affect the practical appicability of the theory in this example, but we focus reader's attention where a care is required.

[60] Which are natural if we consider the linearized problem in $L^{2}$ or $L^{1}$

[61] We note that while decrements $\nu_{3}^{1}$ are small for all the four cases, so the adjoint eigenfunctions look hardly decaying in the $\xi \rightarrow \infty$ asymptotics, the decrements $\nu_{3}^{2}$ are precisely zero, so $\mathbf{W}_{2}$ does not belong to the class $L^{2}$. So in a rigorous treatment of the problem, one would probably need to consider the linearized problem in a suitable subspace of $L^{1}$, so that the adjoint operator would act in its dual, $L^{\infty}$, which is broad enough to contain such non-decaying modes as $\mathbf{W}_{2}$. 